\title{
Tsunami Heights along the Pacific Coast of Northern Honshu Recorded from the 2011 Tohoku and Previous Great Earthquakes
}

\author{
Yoshinobu Tsuji, ${ }^{1}$ Kenji Satake, ${ }^{1}$ Takeo Ishibe, ${ }^{1}$ Tomoya Harada, ${ }^{1,2}$ Aкihito Nishiyama, ${ }^{1}$ and Satoshi Kusumoto ${ }^{1}$
}

\begin{abstract}
The 2011 Tohoku earthquake generated a huge, destructive tsunami with coastal heights of up to $40 \mathrm{~m}$ recorded along northern Honshu. The Sanriku coast experienced similar tsunamis and damage from the 1896 and 1933 Sanriku earthquakes, whereas the only damaging tsunamis on both the Ibaraki and Chiba coasts in the previous century were from the 1960 and 2010 Chile earthquakes. We summarized 12 field surveys in which the height of the 2011 tsunami was recorded at 296 points, along with descriptions of the survey method, reliability, and accuracy. We then compared them with the above-mentioned tsunamis at locations for which specific measurements were given in previous reports. On the Sanriku coast, the 2011 tsunami heights are positively correlated with the previous Sanriku tsunamis, indicating that local variations resulting from the irregular coastline were more dominant factors than the earthquake location, type, or magnitude for near-field tsunamis. The correlations with the Chilean tsunami heights are less significant due to the differences between the local and trans-Pacific tsunamis. On the Ibaraki and Chiba coasts, the 2011 Tohoku and the two Chilean tsunami heights are positively correlated, showing the general decrease toward the south with small local variations such as large heights near peninsulas.
\end{abstract}

Key words: 2011 Tohoku tsunami, 1896 Sanriku tsunami, 1933 Sanriku tsunami, 1960 Chilean tsunami, 2010 Chilean tsunami, Sanriku coast.

\section{Introduction}

On March 11, 2011, a giant earthquake, officially named the "2011 off the Pacific coast of Tohoku earthquake" by the Japan Meteorological Agency

1 Earthquake Research Institute, The University of Tokyo, 1-1-1 Yayoi, Bunkyo-ku, Tokyo 113-0032, Japan. E-mail: satake@eri.u-tokyo.ac.jp

2 Center for Integrated Disaster Information Research (CIDIR), Interfaculty Initiative in Information Studies, The University of Tokyo, 7-3-1 Hongo, Bunkyo-ku, Tokyo 113-0033, Japan.
(JMA), occurred along the Japan Trench where the Pacific plate subducts beneath the Okhotsk plate (Fig. 1). This earthquake, which we refer to as the 2011 Tohoku earthquake in this paper, was the largest earthquake in Japan since the beginning of modern instrumental observations. It had a moment magnitude, $M_{\mathrm{w}}$, of 9.0. It caused 15,883 fatalities and 2,656 people were reported missing (National Police Agency as of August 9, 2013); more than $90 \%$ of the casualties were caused by the tsunami. The maximum tsunami height was nearly $40 \mathrm{~m}$ based on $\sim 5,900$ measurements compiled by the 2011 Tohoku Earthquake Tsunami Joint Survey Group (MoRi et al. 2011, 2012). Such a gigantic earthquake ( M9) was unexpected in Japan, but was the huge tsunami also a surprise?

The Pacific coast of the Tohoku region has suffered from many large tsunamis generated by both near-field and far-field earthquakes. The 1896 Sanriku tsunami caused $\sim 22,000$ casualties, which is more than the 2011 Tohoku earthquake. The 1896 Sanriku earthquake was a "tsunami earthquake" (KANAMORI 1972), that is, one that produces a tsunami that is much larger than that expected from the earthquake magnitude (surface wave magnitude $M_{\mathrm{s}} 7.2$; ABE 1994). The 1933 Sanriku earthquake $\left(M_{\mathrm{s}} 8.5\right)$ also generated significant tsunami damage with $\sim 3,000$ fatalities. The 1960 Chile earthquake was the largest earthquake $\left(M_{\mathrm{w}} 9.5\right)$ of the last century and a transoceanic tsunami struck the Pacific coasts of Japan $\sim 23 \mathrm{~h}$ after the earthquake, causing 142 fatalities. The 2010 Chile earthquake $\left(M_{\mathrm{w}} 8.8\right)$ also generated a trans-Pacific tsunami, which caused property damage to aquaculture facilities such as fishery rafts in Japan. 


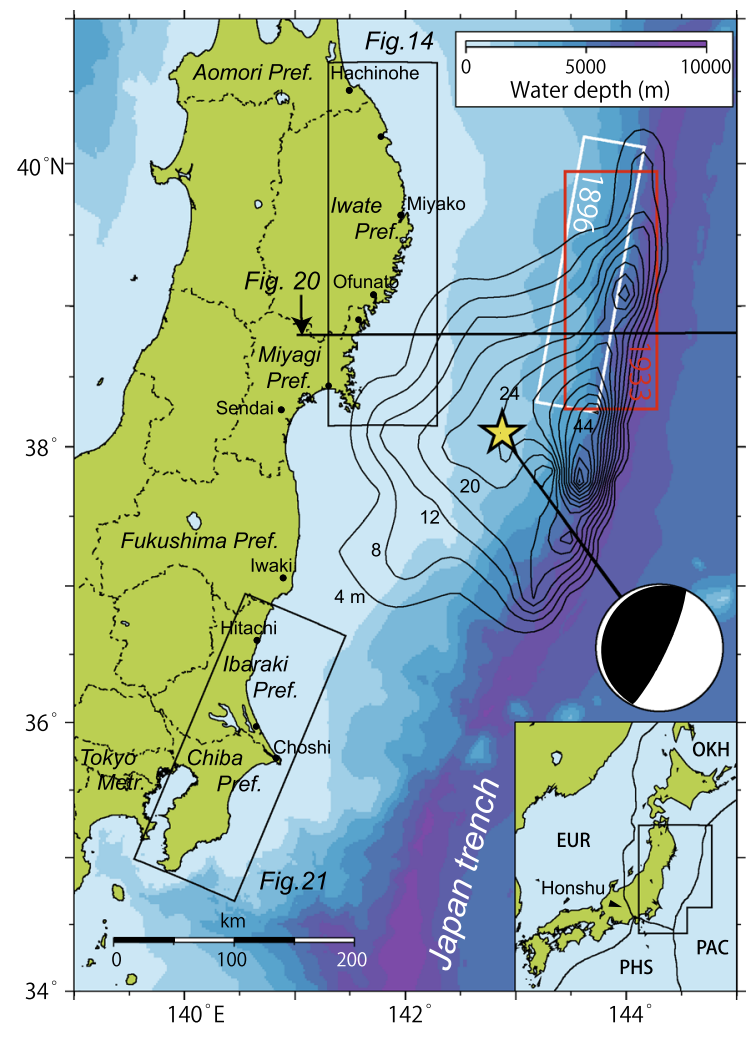

Figure 1

Map of northern Honshu where the 2011 Tohoku earthquake caused tsunami damage. Slip distribution of the 2011 Tohoku earthquake estimated from tsunami waveform inversion (SATAKE et al. 2013) is shown by contours with 4-m intervals. The yellow star indicates the epicenter of the mainshock, as determined by the JMA. The focal mechanism is provided by the Global Centroid Moment Tensor project. The black rectangles indicate regions shown in Figs. 14 and 21 . The white and red rectangles indicate fault models of the 1896 (TANIOKA and SENo 2001) and 1933 Sanriku (AIDA 1977) earthquakes, respectively. Dashed lines indicate prefectural boundaries. The location of the profile in Fig. 20 is also shown. In the inset, OKH, EUR, PAC, and PHS indicate the Okhotsk, Eurasia, Pacific, and Philippine Sea Plates, respectively

This paper first summarizes our field surveys (Tsuj et al. 2011) in which the tsunami heights of the 2011 Tohoku earthquake were recorded, along with descriptions of the survey method, reliability, and accuracy. Then, we compare the tsunami heights with those from previous earthquakes both near Japan and across the Pacific Ocean. For the Sanriku coast, we compare the 2011 tsunami heights and inundation areas with two local tsunamis (i.e., the 1896 and 1933 Sanriku tsunamis) and two trans-Pacific tsunamis (i.e., 1960 and 2010 Chilean tsunamis) at selected sites where direct comparisons can be made. For the Pacific coasts of Ibaraki and Chiba prefectures, we similarly compare the 2011 tsunami heights with the 1960 and 2010 Chilean tsunami heights because no damaging tsunamis were recorded from near-field earthquakes in the last century. On the central Sanriku coast, the sawtooth coastal topography is a major factor that strongly affects tsunami heights more so than the earthquake location, type, or magnitude. On the other areas of the Sanriku coast, and the Ibaraki and Chiba coasts, local variations are smaller and the general pattern of tsunami heights reflects the location, slip distribution, and magnitude of the parent earthquake.

\section{Field Surveys of Tsunami Heights from the 2011 Tohoku Earthquake}

We conducted 12 field surveys between March 16 and October 24, 2011. Locations and tsunami heights above sea level were generally measured with handheld GPS receivers and auto-levels, laser rangefinders, or total stations. In the surveys, we first sought traces that indicate the tsunami heights, which were classified as inundation heights, runup heights, and tsunami heights in ports. For tsunami inundation, we measured flow depths above ground based on watermarks or other physical evidence. The highest inundation on a slope where the flow velocity is considered to have been zero is classified as the runup height. Runup heights were measured from debris carried by the tsunami, the absence of leaves, or similar evidence on a slope. However, as most physical evidence had disappeared by June 2011, later surveys mainly relied on eyewitness accounts. In ports where the tsunami did not inundate above the wharfs, we measured tsunami heights based on eyewitness accounts and classified them as tsunami heights in ports, as proposed by Tsus et al. (2010).

The 2011 tsunami heights in this paper are heights that were above sea level at the time of the maximum tsunami arrival. The measured heights were corrected for differences in tide levels between the measurement time and the arrival time of the maximum tsunami. The arrival times of the maximum tsunami at Hachinohe, Miyako, Kamaishi, Ofunato, Ayukawa, 
(a)

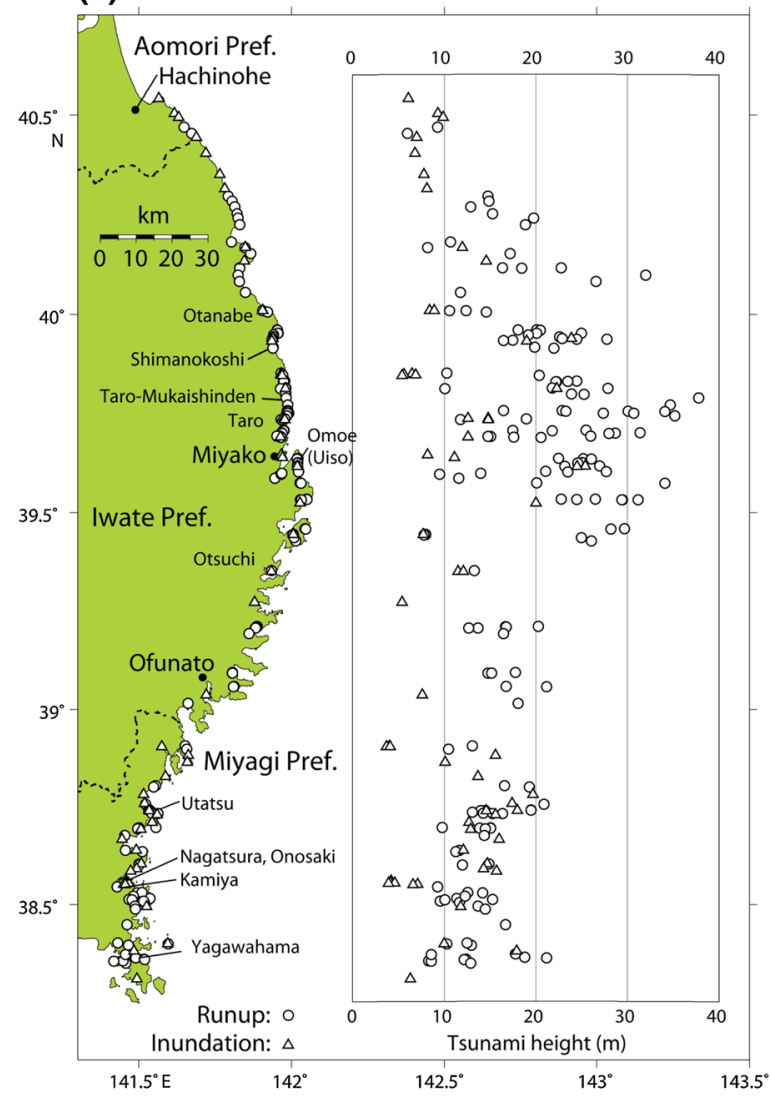

(b)

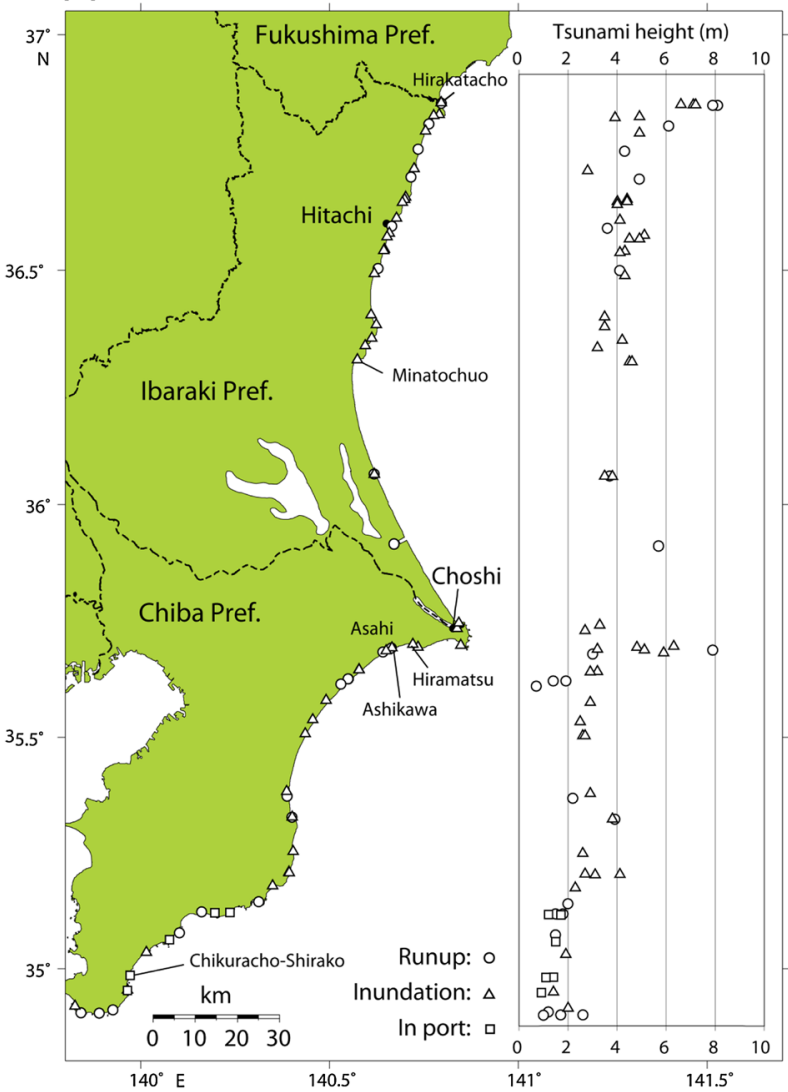

Figure 2

a Distribution of tsunami heights in Aomori, Iwate, and Miyagi prefectures (Tsuj et al. 2011). Circles and triangles indicate runup and inundation heights, respectively. The dashed lines indicate prefectural boundaries. b Distribution of tsunami heights in Ibaraki and Chiba prefectures. Circles, triangles, and squares indicate runup heights, inundation heights, and tsunami heights in ports, respectively

Onahama, Oarai, Choshi Fishing Port, and Mera (JMA 2011) were used for tide correction. At some locations where the measurement points were far from the sea, we measured altitudes above Tokyo Peil (TP), the leveling datum of Japan. In such cases, we considered land subsidence due to the mainshock, which was as large as $1 \mathrm{~m}$, as recorded by continuous GPS data (OzAwa et al. 2011). The altitudes were converted to heights above mean sea level, then to heights above tide level at the time of the maximum tsunami arrival.

The reliability of the evidence was categorized into three classes (A, B, and $\mathrm{C}$ ), as some tsunami heights were obtained from clear watermarks, whereas others were based on less objective eyewitness accounts (e.g., Shuto and Unohana 1984). Class A indicates the most reliable data, which are based on clear physical evidence or objective eyewitness accounts. Class B indicates evidence based mostly on natural traces such as leaves, grass roots, or debris; while class $\mathrm{C}$ indicates the least reliable data that are based on equivocal evidence such as fishing floats in trees or broken windowpanes. Other catalogs such as the NOAA/WDC Tsunami runup Database introduce another type of validity (doubtful), without classifying the reliability.

The measurement accuracy was also categorized into three classes (a, b, and c). Class a means measurement errors are considered to be $<0.2 \mathrm{~m}$. The error for class $b$ ranges $0.2-0.5 \mathrm{~m}$ because repeated measurements were performed using an auto-level or the sea was rough at the time of measurement. Class $\mathrm{c}$ means the errors are $>0.5 \mathrm{~m}$ because the sea level could not be directly measured or laser measurements were performed without a reflector. 


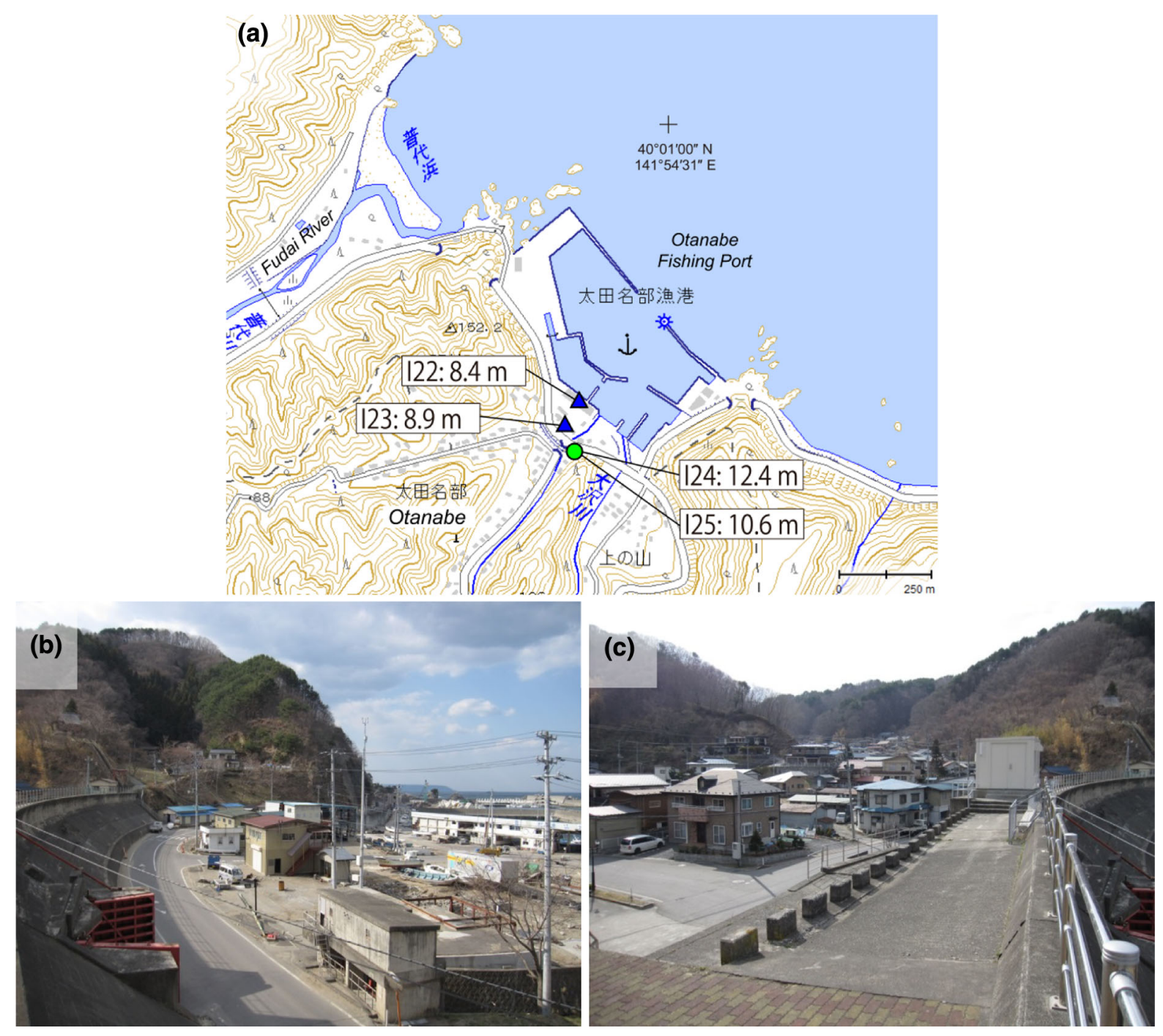

Figure 3

a Tsunami heights at Otanabe (Otanabe Fishing Port), Fudai Village, Iwate Prefecture (with 1:25,000 digital topographic map added from the Geospatial Information Authority of Japan). Circles and triangles indicate runup and inundation heights, respectively. Closed circles and triangles are color coded according to tsunami heights (i.e., 0-10 m blue; 10-20 m green, 20-30 m yellow, 30 m or above red). b Destructive damage outside the coastal levee (Otanabe Fishing Port). c Very minor damage inside the coastal levee (residential district)

\section{Tsunami Heights from 2011 Tohoku Earthquake}

Measurements of the 2011 tsunami were made at 296 points on the Sanriku coasts of Aomori (Northern Sanriku), Iwate (Central Sanriku), and Miyagi (Southern Sanriku) prefectures, and the Pacific coasts of Ibaraki and Chiba prefectures (Fig. 2a, b). While the details were reported by Tsus et al. (2011) with the survey points shown on 1:25,000 maps from the
Geospatial Information Authority of Japan and photographs of measured tsunami traces, we summarize the tsunami heights at some typical locations. In this paper, we employ the measurement numbers given by Tsuj et al. (2011), which start with a letter indicating the prefecture (i.e., A, I, M, B, and C for Aomori, Iwate, Miyagi, Ibaraki, and Chiba prefectures, respectively) and are followed by the sequence number in each prefecture. 

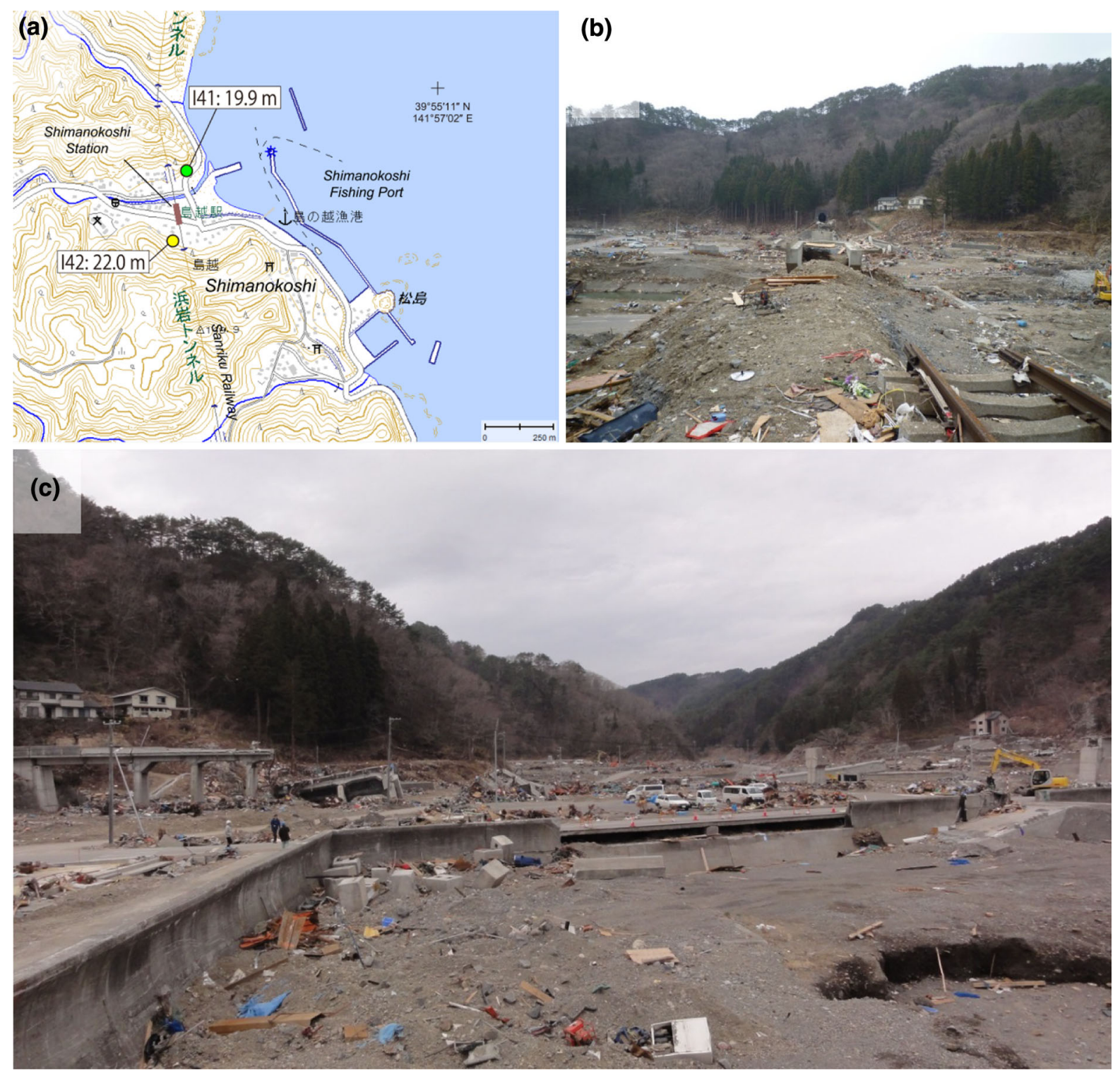

Figure 4

a Tsunami heights at Shimanokoshi, Tanohata Village, Iwate Prefecture. The symbols and their meanings are the same as in Fig. 3. b, c View of tsunami damage at Shimanokoshi

\subsection{Sanriku Coast}

Along the northern Sanriku coast of Aomori Prefecture, five tsunami heights ranging 6.0-9.9 were measured. Along the central Sanriku coast of Iwate Prefecture, 136 tsunami heights were measured. Tsunami heights were mostly over $10 \mathrm{~m}$, while they were above $30 \mathrm{~m}$ at 10 measurement points. Along the southern Sanriku coast of Miyagi Prefecture, 76 tsunami heights were measured. The tsunami heights were mostly $10-20 \mathrm{~m}$, slightly lower than those in Iwate Prefecture, indicating that the highest tsunami height was not recorded directly landward of the largest slip region near the Japan Trench (Figs. 1, 2a).

In the Otanabe district of Fudai Village, a 15-mhigh coastal levee had been constructed between the fishing port area and residential area (Fig. 3). The inundation heights were measured as 8.4 and $8.9 \mathrm{~m}$ in the fishing port area, and two runup heights of $10.6 \mathrm{~m}$ 


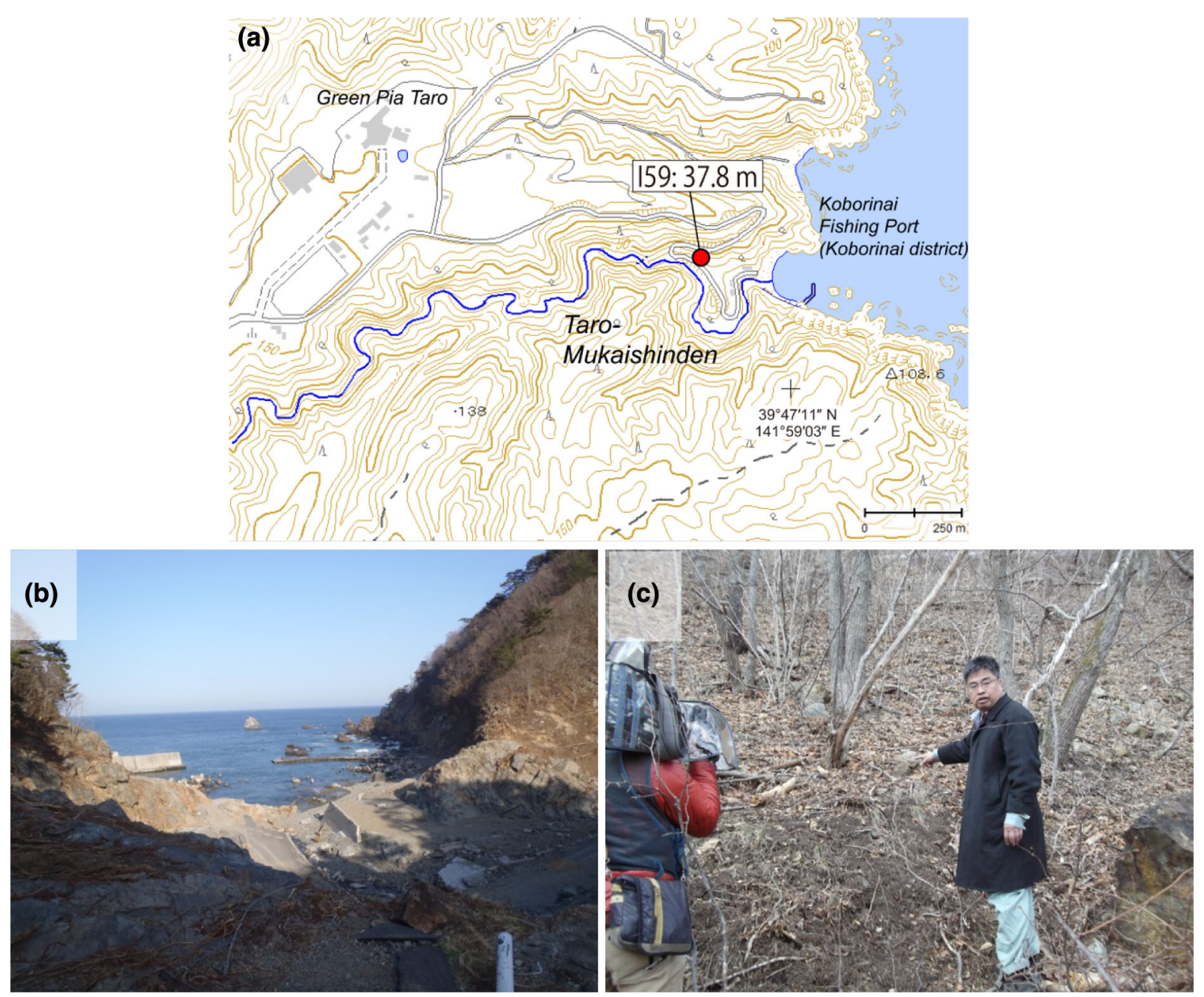

Figure 5

a Tsunami height at Taro-Mukaishinden, Miyako City, Iwate Prefecture. The symbol and its meaning are the same as in Fig. 3. b View of tsunami damage at Taro-Mukaishinden. The white pole in the lower-right of the picture was bent by the tsunami. $\mathbf{c}$ The survey point where the largest tsunami runup height of $37.8 \mathrm{~m}$ in our field surveys was measured

(class B) and $12.4 \mathrm{~m}$ (class C) were measured on the coastal levee (I22-I25). The floodgate, which was closed before the arrival of tsunami, completely protected the residential area from the devastating tsunami, while the outside fishing port area was severely damaged.

At Shimanokoshi in Tanohata Village, a bridge and Shimanokoshi Station of the Sanriku Railway were completely destroyed by the tsunami, and all the houses were swept away or leveled except for two that were located on a hill (Fig. 4). A runup height of $19.9 \mathrm{~m}$ was measured at the northern slope near the dai-ni (second) Shimanokoshi Tunnel (I41). A large amount of wood building materials and debris was deposited throughout the tunnel. The runup height of $22.0 \mathrm{~m}$ was measured at the front yard of one of the surviving houses (I42).

At Taro-Mukaishinden near Koborinai Fishing Port in Miyako City, a runup height of $37.8 \mathrm{~m}$ (I59), the maximum height in our field surveys, was measured on the basis of the upper limit of debris and absence of leaves (Fig. 5). Three firefighters, who were advising fishermen to evacuate, were killed at an elevation of about $30 \mathrm{~m}$.

At Taro in Miyako City, coastal levees with a height of $10 \mathrm{~m}$ and a total length of $\sim 2.4 \mathrm{~km}$ had 


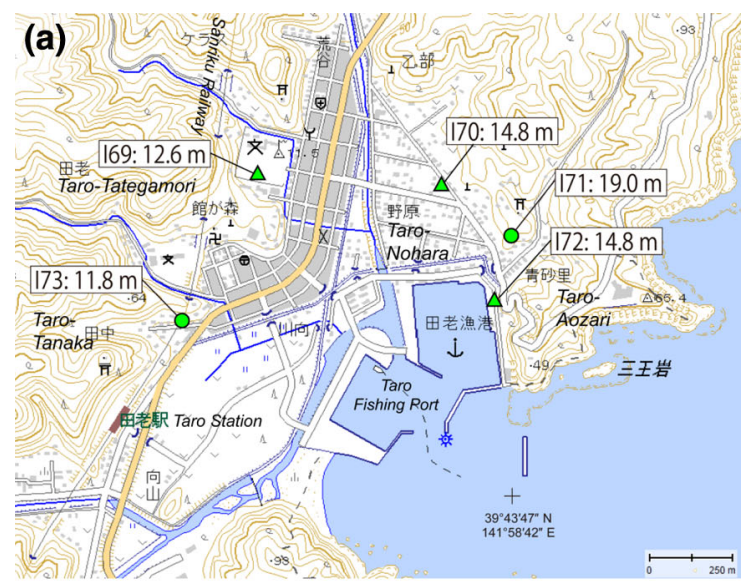

(b)

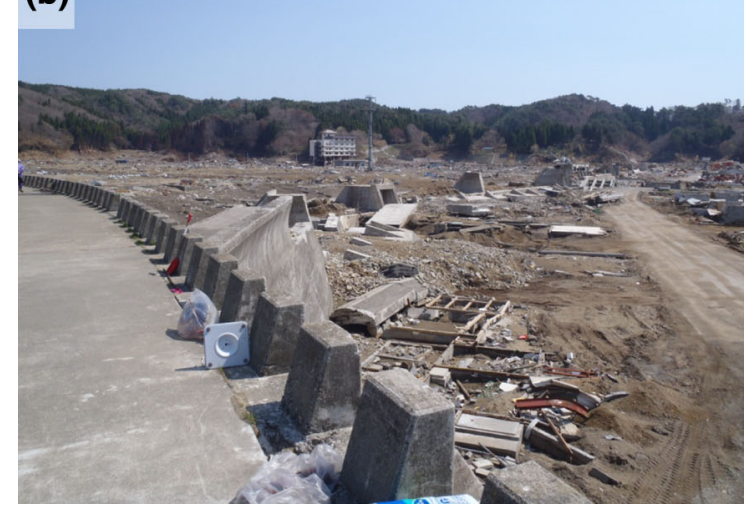

Figure 6

a Tsunami heights at Taro-Tategamori (I69), Taro-Nohara (I70), Taro-Aozari (I71 and I72), and Taro-Tanaka (I73), Miyako City, Iwate Prefecture. The symbols and their meanings are the same as in Fig. 3. b Part of the breakwater destroyed by the 2011 tsunami. The building in the background is a hotel that the tsunami damaged up to the third floor $(14.8 \mathrm{~m}$; I70)

been constructed; however, the 2011 tsunami destroyed a portion of these coastal levees, and transported blocks and other debris from the structure more than $100 \mathrm{~m}$ inland (Fig. 6). Almost all houses and fishing facilities were swept away or were completely leveled. The first three floors of a hotel were severely damaged, indicating an inundation height of $14.8 \mathrm{~m}$ (I70). Tsunami traces on the wall of a Japan Fisheries Cooperatives icehouse indicate the same inundation height (I72). Runup heights of $11.8 \mathrm{~m}$ (I73) and $19.0 \mathrm{~m}$ (I71) were measured at the western and eastern part of the residential area, respectively. Tsunami trace at a middle school indicates an inundation height of $12.6 \mathrm{~m}$ (I69).
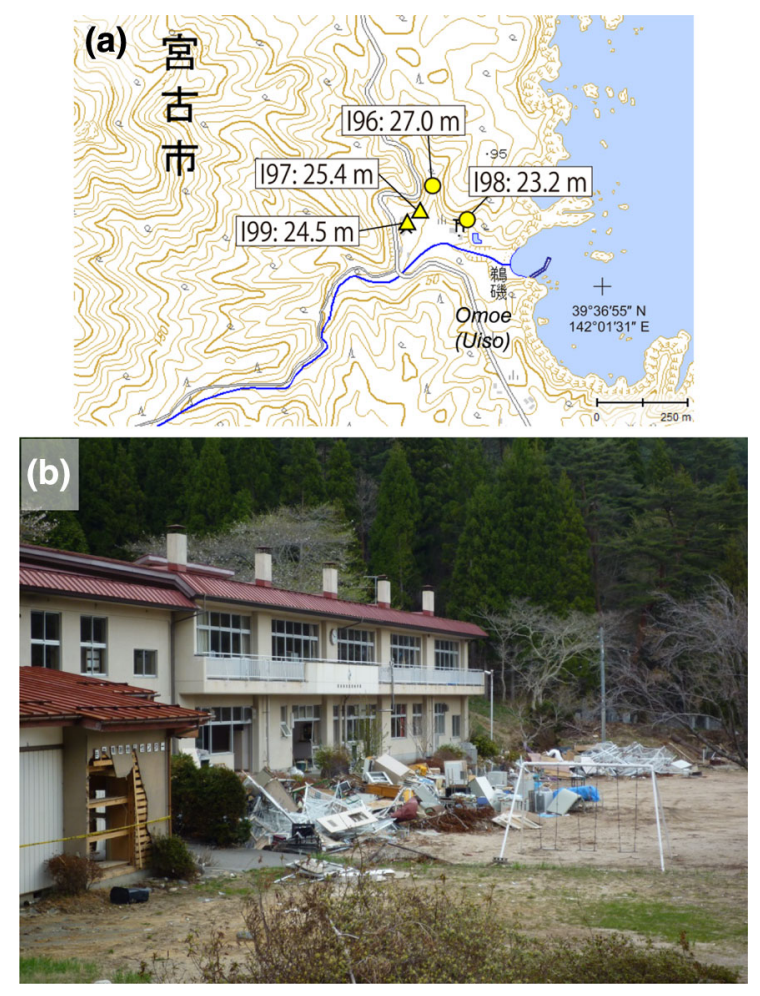

Figure 7

a Tsunami heights at Omoe (Uiso), Miyako City, Iwate Prefecture. The symbols and their meanings are the same as in Fig. 3. b View of tsunami damage at Uiso Elementary School

At Omoe (Uiso), which is located on the east coast of Miyako City on the Omoe Peninsula, the tsunami arrived at Uiso Elementary School, which is sited on a hill having an altitude of $>20 \mathrm{~m}$ (Fig. 7). Panes of glass on the first floor were broken and a large amount of debris was scattered over the school playground. The measured inundation heights were 25.4 and $24.5 \mathrm{~m}$, while the runup heights were 27.0 and $23.2 \mathrm{~m}$ (I96-I99).

At Utatsu-Namiita and Utatsu-Minato in Minamisanriku Town, most houses in the lowlands were leveled or swept away (Fig. 8). It was found from eyewitness accounts that the second tsunami arrival was the largest. At the northern settlement, one inundation height of $18.0 \mathrm{~m}$ and two runup heights of 19.4 and $19.5 \mathrm{~m}$ were measured (M15-M17). Although the southern settlement is located nearby, a lower inundation height of $14.6 \mathrm{~m}$ and runup heights of 14.5 and $14.0 \mathrm{~m}$ were measured (M18M20). 


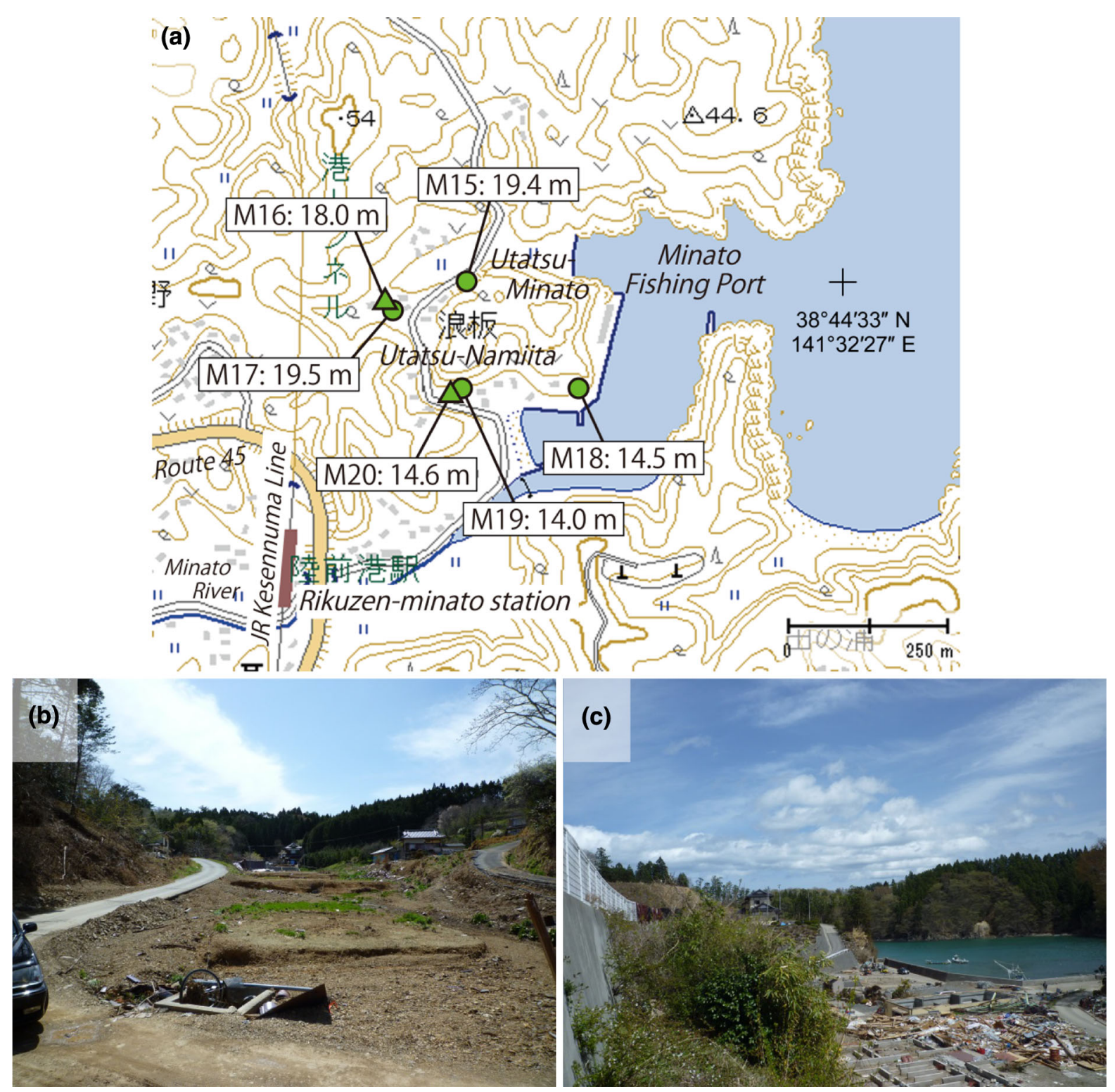

Figure 8

a Tsunami heights at Utatsu-Namiita (M15, M16, and M17) and Utatsu-Minato (M18, M19, and M20), Minamisanriku Town, Miyagi Prefecture. The symbols and their meanings are the same as in Fig. 3. b, c Tsunami damage at Utatsu-Namiita (left) and Utatsu-Minato (right)

Around Nagazuraura, which is a brackish lake connected to Oppa Bay in Ishinomaki City, almost all the houses were inundated by the 2011 tsunami (Fig. 9). Japanese black pines and houses at the mouth of the Kitakami River were swept away. Many houses and rice paddies were submerged due to significant ground subsidence, and sand was deposited all over the residential district. In Onosaki district, which is located eastward of Nagazuraura, inundation heights of 4.2 and $3.9 \mathrm{~m}$ were measured (M43, M46). In Nagatsura district to the west of Nagazuraura, four inundation heights ranging 4.1-7.1 m were obtained (M44, M45, M47 and M48). Some residents drowned at the temple to which they had evacuated, erroneously assuming it was at a safe elevation.

At Okawa Elementary School in Kamaya, Ishinomaki City, 10 teachers and 74 of the 108 students 


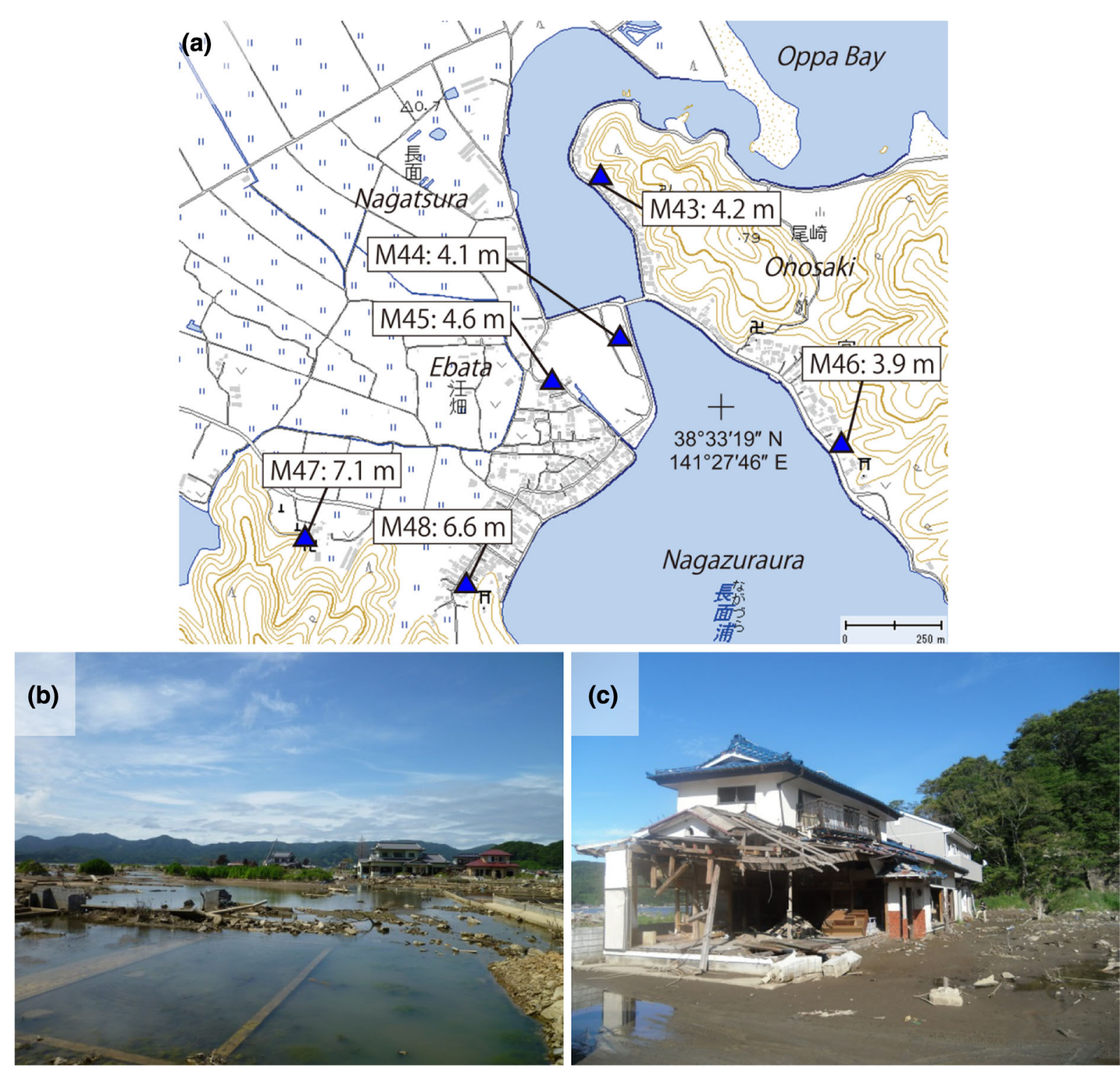

Figure 9

a Tsunami heights at Onosaki (M43, M46) and Nagatsura (M44, M45, M47, and M48), Ishinomaki City, Miyagi Prefecture. The symbols and their meanings are the same as in Fig. 3. b, c View of tsunami damage and ground subsidence at Onosaki and Nagatsura

died on the way to an evacuation site. It was found that the tsunami, which had run up the Kitakami River, inundated to a height above the ceiling of the second floor of the school (Fig. 10). A runup height of $9.3 \mathrm{~m}$ was measured at a slope behind the school (M49).

At Yagawahama in Ishinomaki City, almost all the houses were swept away by the tsunami, and farmland was covered by tsunami sediment deposits that included sand, shell fragments and gravel (Fig. 11). A large amount of debris, which was caught in trees on the side of a road, indicate an inundation height of $18.7 \mathrm{~m}$ (M67). The maximum runup height of $21.2 \mathrm{~m}$ in the Miyagi Prefecture was measured at the cemetery behind Tofuku-ji Temple (M68).

\subsection{Ibaraki and Chiba Coasts}

On the Ibaraki coast (between $35.7^{\circ}$ and $36.9^{\circ} \mathrm{N}$ ), 36 measurements of the 2011 Tohoku tsunami ranged $2.8-8.1 \mathrm{~m}$, and the heights generally decreased 


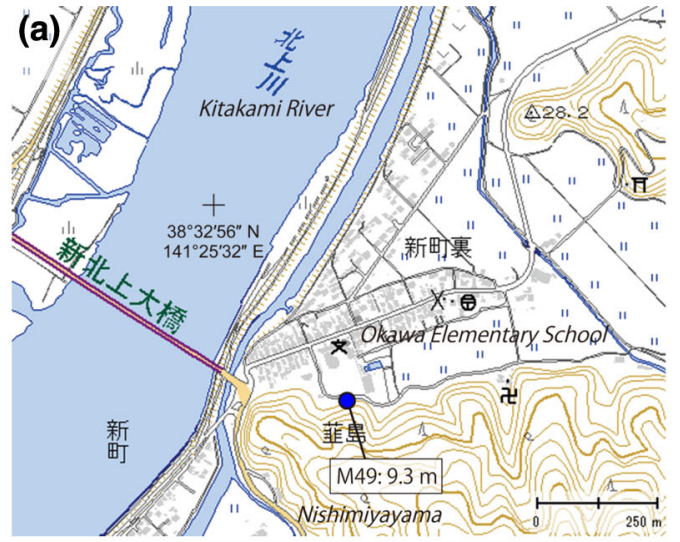

(b)

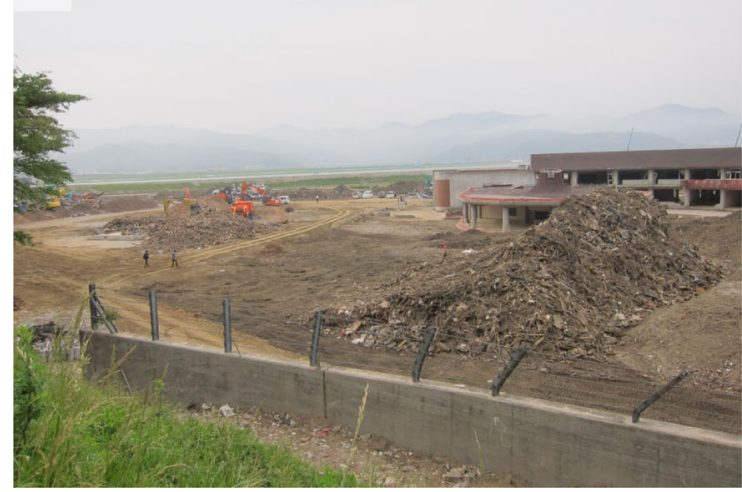

Figure 10

a Tsunami height at Kamaya in Ishinomaki City, Miyagi Prefecture. The symbol and its meaning are the same as in Fig. 3.

b View of tsunami damage at the Okawa Elementary School

toward the south (Fig. 2b). The typical tsunami height was $4 \mathrm{~m}$ and the local variations were significantly smaller than those along the Sanriku coast. Along the coast of Chiba Prefecture (between $34.9^{\circ}$ and $\left.35.7^{\circ} \mathrm{N}\right), 43$ measurements of the 2011 tsunami heights were generally $<4.0 \mathrm{~m}$. They gradually decreased toward the south, and most tsunami heights were $<2 \mathrm{~m}$ along the coast of southern Chiba Prefecture. However, tsunami heights were locally high (5.1-7.9 m) around Asahi City.

At Hirakatacho in Kitaibaraki City, the fishing port and residential area were severely damaged (Fig. 12). The first floors of many houses were destroyed by the tsunami and some houses collapsed completely. Watermarks on buildings indicated inundation heights ranging $6.6-7.2 \mathrm{~m}$, while debris indicated runup heights of 7.9 and $8.1 \mathrm{~m}$ (B1-B5).
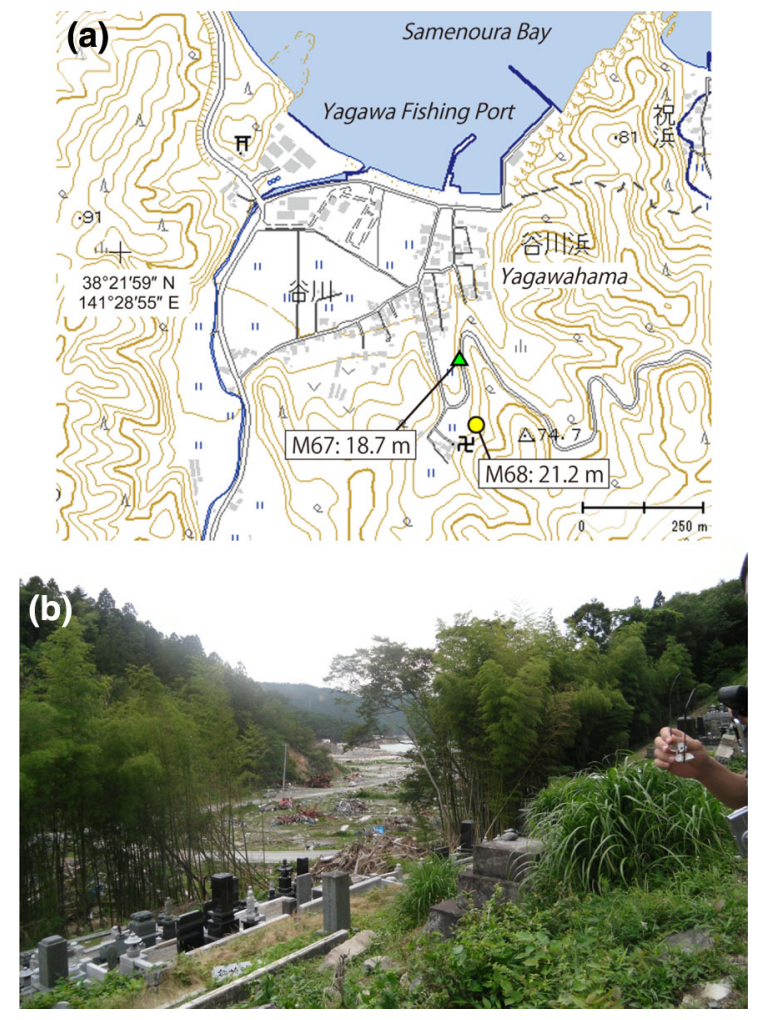

Figure 11

a Tsunami heights at Yagawahama, Ishinomaki City, Miyagi Prefecture. The symbols and their meanings are the same as in Fig. 3. b View from the survey point with a runup height of $21.2 \mathrm{~m}$ (M68)

At Minatochuo in Oarai Town, which is located in the central part of Ibaraki Prefecture, inundation heights of 4.5 and $4.6 \mathrm{~m}$ were measured from the watermarks on the ferry terminal building (B30, B31).

At Asahi City, the tsunami heights were locally high. Residential areas along the Pacific coast were badly damaged and 13 people were killed (Fig. 13). In the Hiramatsu district where buildings were densely distributed, a clear watermark on the windows of a store indicated the inundation height as $6.3 \mathrm{~m}$ (C4). In the Ashikawa district, an inundation height of $5.1 \mathrm{~m}$ and a runup height of $7.9 \mathrm{~m}$ were measured (C5, C6). At the Shirako Fishing Port in Chikuracho-Shirako in Minamiboso City, according to eyewitness accounts, the first tsunami arrived at 15:15-15:30 and the seawater rose to near the top of the quay. The measured tsunami heights in this port ranged 1.0-1.4 $\mathrm{m}(\mathrm{C} 35, \mathrm{C} 36)$. The eyewitnesses also 


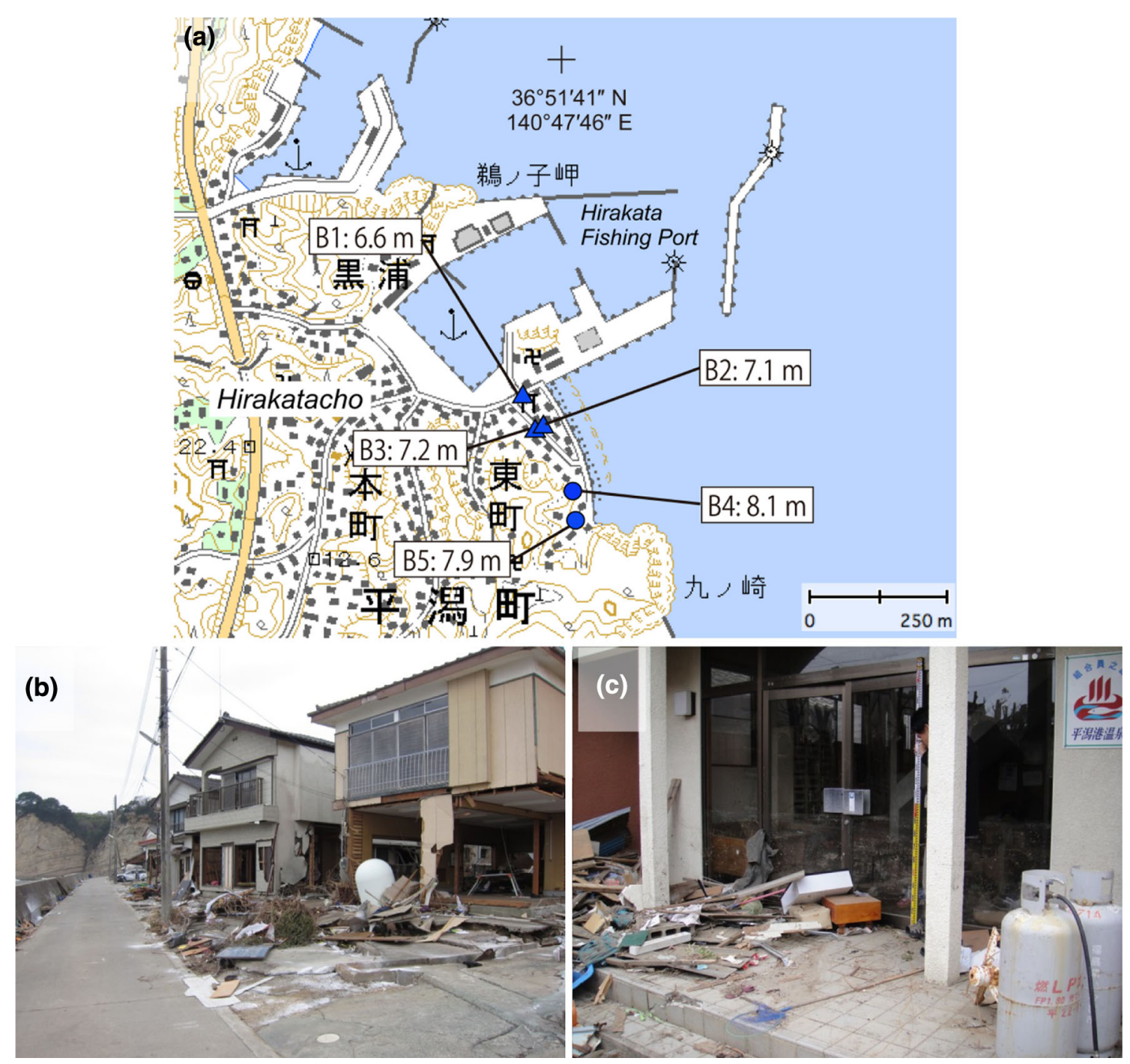

Figure 12

a Tsunami heights at Hirakatacho, Kitaibaraki City, Ibaraki Prefecture. The symbols and their meanings are the same as in Fig. 3. b View of tsunami damage at Hirakatacho. c Watermarks on windowpanes of entrance doors of the guesthouse Yanagiya (0.89 m above ground level,

B3)

reported that the second tsunami arrival was one hour after the first arrival and that the height was similar.

\section{Tsunami Height Data from Past Earthquakes}

\subsection{6 and 1933 Sanriku Earthquakes}

A number of field surveys and investigations have been conducted for the tsunamis caused by the June 15, 1896 and March 3, 1933 Sanriku earthquakes to determine the heights and inundation areas (e.g., Imamura 1934; Shuto and Goto 1985a, b; ImAmura and Watanabe 1990; Hatori 1995). In this study, we examined the reports of the original surveys published soon after the earthquakes (i.e., in the 1890s and 1930s), rather than secondary or recent papers. We briefly describe these original reports below.

YAMANA (1896; reproduced by UnOHANA and OTA 1988) reported that his survey was conducted between July 28 and September 9, 1896, in all the 


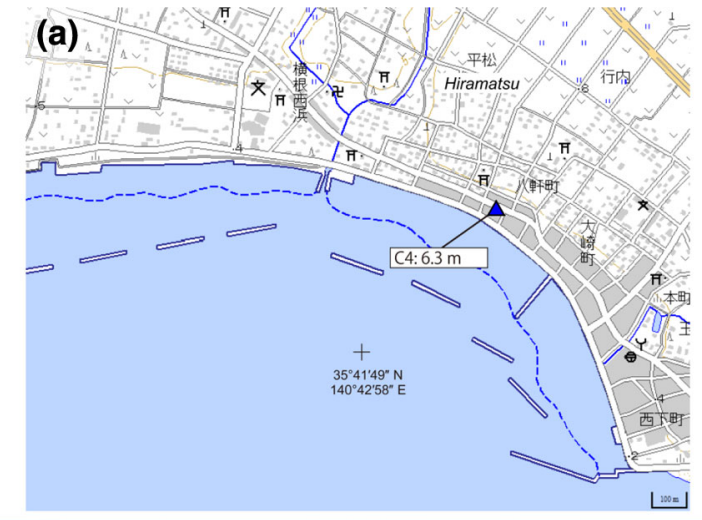

(b)

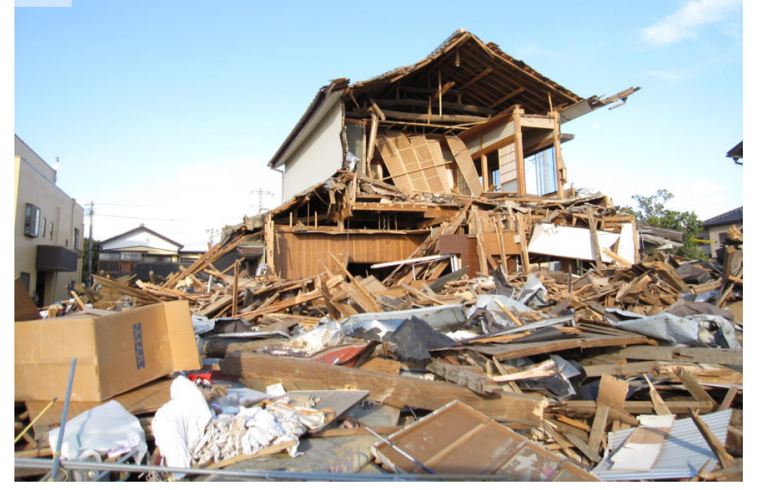

Figure 13

a Tsunami height at Hiramatsu, Asahi City, Chiba Prefecture. The symbol and its meanings are the same as in Fig. 3. b A damaged house in Hiramatsu district

villages along the Sanriku coast. He reported not only tsunami heights, but also casualty numbers and property damage for each village, along with providing 168 maps illustrating the disaster. The largest heights of $180 \mathrm{shaku}$, or $55 \mathrm{~m}(1$ shaku $=0.303 \mathrm{~m})$ were reported at Kosode (I12, I13) and RyoriShirahama (I133). However, the accuracy of Yamana's measurements varies at different locations. For example, the reported runup height is 132 shaku $(40.0 \mathrm{~m})$ at Shimanokoshi (I41, I42), whereas the height is $130-180$ shaku (39-55 m) at Ryori-Shirahama (I133).

IKI (1897) surveyed the Sanriku coast from June 20 to July 21, 1896, and his report contains list of locations and tsunami heights. However, it contains very few figures, making it difficult to identify the exact measurement locations. The reported tsunami heights in feet $(1$ foot $=0.305 \mathrm{~m})$ were from sea level at the time of measurement. He measured tsunami heights from changes in vegetation color or watermarks due to tsunami inundation, which are considered to be highly reliable, or from debris or scratches on trees. He also distinguished heights measured by himself and those based on eyewitness accounts.

Matsuo (1933) reported tsunami heights from the 1896 and 1933 Sanriku tsunami based on surveys he conducted during March 3-10 and May 19-June 4, 1933, with the support of the Iwate and Miyagi prefectural governments. The tsunami heights from the 1896 Sanriku tsunami were measured 37 years after the event, based on eyewitness accounts. The often quoted maximum height of $38 \mathrm{~m}$ at RyoriShirahama (I133) from the 1896 Sanriku tsunami was based on this report. MATsuo (1934) also reported the 1933 tsunami heights and inundation areas of the 1896 and 1933 tsunamis along the coasts of Hokkaido and Aomori prefectures.

Kunitomi (1933) reported tsunami heights together with arrival times from both eyewitness accounts and tide gauge records, the number of tsunami waves, and periods from the 1933 Sanriku tsunami in Hokkaido, along the Sanriku coasts of Aomori, Iwate, Miyagi prefectures, and along the Pacific coast of Fukushima Prefecture, based on field surveys from meteorological observatories. Tsunami heights were measured on the basis of traces left at the coast and/or eyewitness accounts. Appendix figures indicating tsunami heights and inundation areas on maps with a scale of 1:200,000, and photographs recording severe damage are also shown. Kunitomi (1933) also compared the 1933 heights with the 1896 heights from IKI (1897) and surveys from the Civil Engineering Division of the Iwate prefectural government.

The Earthquake Research Institute (1934) reported the survey results for the Pacific coasts from Hokkaido to Ibaraki Prefecture. It reported tsunami heights with maps showing estimated inundation limits, damage to houses and other structures, relations between the severity of tsunami effects and topographic conditions, photographs, and tide gauge records. In addition, ОтUкA (1934) discussed the relationship between tsunami heights and the topography of the Sanriku coast. 

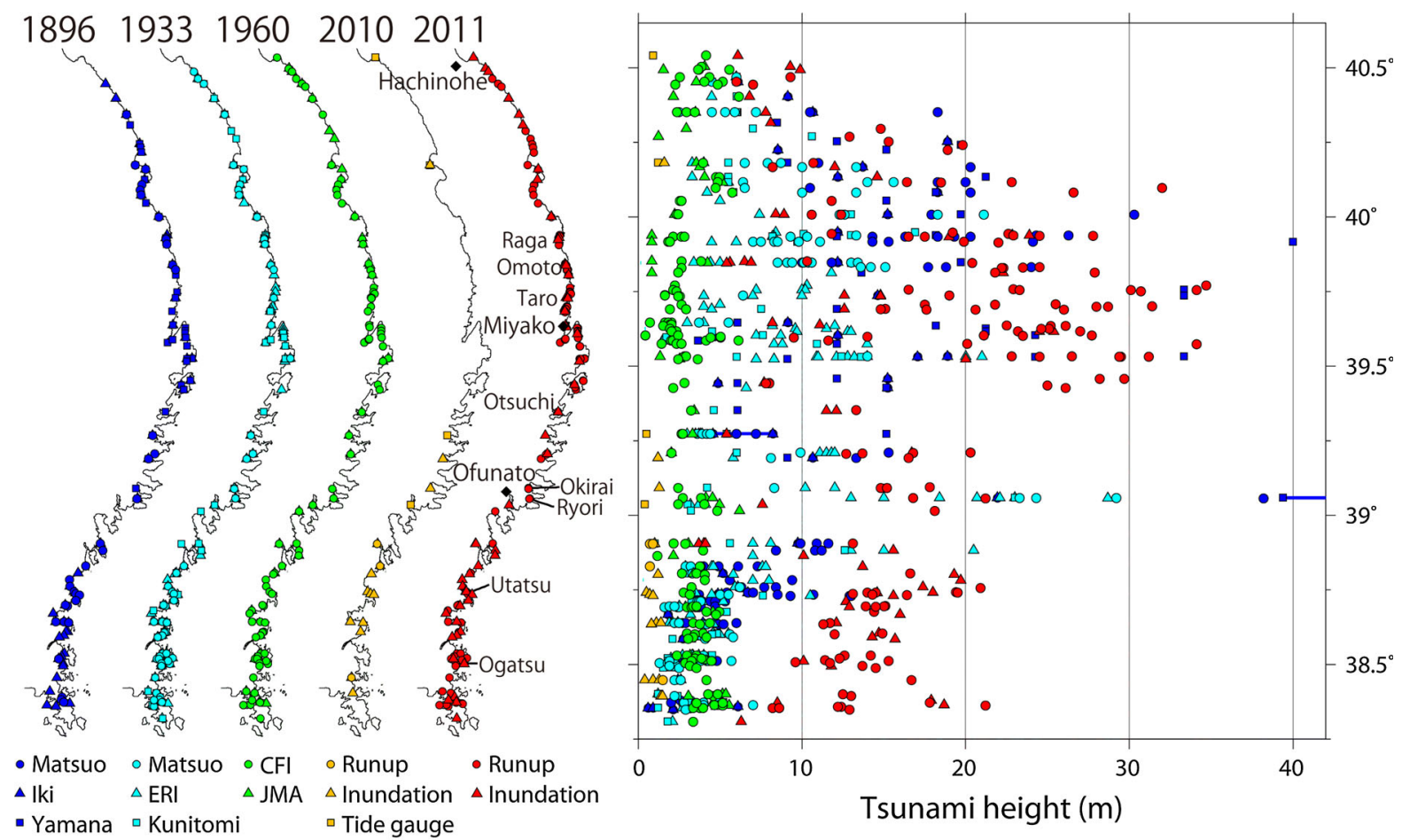

Figure 14

Comparison of 1896 and 1933 Sanriku tsunami heights, 1960 and 2010 Chilean tsunami heights with 2011 tsunami heights. Bars indicate the range of measured heights. The measurement locations are indicated on the maps on the left. Only data measured at the same locations for two or more tsunamis are shown. Blue circles, triangles, and squares, respectively, indicate the 1896 tsunami heights given by MATsuO (1933), IKI (1897), and Yamana (1896), which were reproduced by Unohana and Ота (1988). Light blue circles, triangles, and squares, respectively indicate the 1933 heights from Matsuo (1933, 1934), the EarthquaKe Research Institute (1934), and Kunitomi (1933). Green circles and triangles indicate the 1960 Chilean tsunami heights from CFI (1961), and JMA (1961), respectively. Orange circles, triangles, and squares, respectively, indicate the 2010 runup, inundation heights, and heights from tide gauges by Tsuj et al. (2010). Red circles and triangles, respectively, indicate the 2011 runup and inundation heights from Tsus et al. (2011)

\subsection{0 and 2010 Chile Earthquakes}

The Committee for Field Investigation of the Chilean Tsunami of 1960 (CFI, 1961) consisted of 100 investigators who conducted surveys along the entire Pacific coast of Japan from Hokkaido to Kyushu. They compiled measured tsunami heights above TP (nearly equal to mean sea level), reliability (1-5), and arrival time of the maximum tsunami. The report contains detailed maps showing the exact measurement locations.

Japan Meteorological Agency (JMA, 1961) contains field survey results made by the agency. The reported tsunami heights were either measured on tide gauges or were based on eyewitness accounts. The definition of tsunami heights and datum varies according to location; and detailed maps indicate measurement locations.
IMAI et al. (2010) reported tsunami heights from the 2010 Chile earthquake along the coasts of the Kanto and Tokai districts measured from field surveys or tide gauges. Tsus et al. (2010) reported the 2010 tsunami heights along the Sanriku coast measured from field surveys or tide gauges. The tsunami heights were above sea level at the time of maximum tsunami and were classified as inundation heights, runup heights, and tsunami heights in ports. The survey locations were measured using handheld GPS devices showing latitudes and longitudes.

\subsection{Selection of Locations for Comparison}

We selected locations for which measurements for the 2011 tsunami and at least one of the previous tsunamis were available in order to enable a direct comparison. While the precise measurement points of 
the 1896 and 1933 Sanriku tsunamis are unknown, comparisons were made at locations (village or smallest bay). We attempted to reduce the local variability in tsunami heights as much as possible. For the 1960 and 2010 Chilean tsunamis, we selected tsunami height data measured in the same ports or similar coastal locations by examining maps of the measurement points. For the comparison of tsunami heights between the 2011 and previous tsunamis, we use the median height of multiple measurements in each report. For an example, three tsunami heights (by the Earthquake Research Institute, median of five heights by Matsuo, and by Kunitomi) were compared with the 2011 height $(7.8 \mathrm{~m})$ at $\mathrm{I} 3$ (Taneichi, Hirono Town). For another example, the median height of three measurements of the 2011 tsunami (I16-I18) was compared with the previous tsunamis at the Noda Fishing Port in Noda Village.

\section{Comparison of Tsunami Heights on the Sanriku Coast}

Along the Sanriku coasts of Aomori, Iwate, and Miyagi prefectures, Tsus et al. (2011) measured 217 tsunami heights at 120 locations. Of these, the 1896 and 1933 heights were reported at 80 and 94 locations, respectively (Fig. 14; Table 1). The 1896 tsunami heights are smaller than the 2011 tsunami heights (median ratio is 0.69; Table 3), and the 1933 heights are even smaller (median ratio 0.33). The 1960 and 2010 Chilean tsunami heights can be compared with the 2011 tsunami heights at 98 and 19 locations, respectively (Table 2). The 1960 tsunami heights are also smaller than the 2011 tsunami heights (median ratio 0.25), and the 2010 heights are much smaller (median ratio 0.07).

\subsection{Central Sanriku Coast}

Along the central Sanriku coast with latitudes between $39.0^{\circ}$ and $40.2^{\circ} \mathrm{N}$, the 2011 tsunami heights ranged from 5 to $40 \mathrm{~m}$ (Fig. 14). In this region, the 1896 heights were approximately similar (median ratio is 0.85 ; Table 3) to the 2011 heights, whereas the 1933 heights were smaller (median ratio 0.47). The 1960 and 2010 Chilean tsunami heights were much smaller than the 2011 heights (median ratio is 0.16 and 0.09 , respectively).

At Raga in Tanohata Village, the 1896 and 2011 tsunami heights were much larger than the 1933 tsunami height. The measured 2011 tsunami heights ranged from 23 to $28 \mathrm{~m}$ (I34-I37), while the reported 1896 tsunami heights varied from 60 shaku $(18 \mathrm{~m}$; YAMANA 1896), 75 feet (23 m; IKI 1897), and 24-26 m (Matsuo 1933). During our survey, a local resident testified that the 1896 Sanriku tsunami washed away their former family house, which was at the same location as the current one, whereas the 2011 tsunami did not. Furthermore, the 2011 tsunami inundation limit is located just below the tsunami stone transported by the 1896 tsunami (Fig. 15). TAKEDA (1987) measured the level of the tsunami stone as $28.2 \pm 1.2 \mathrm{~m}$ above TP and concluded that the maximum runup height of the 1896 Sanriku tsunami at Raga may have been higher than $30 \mathrm{~m}$. The 1933 tsunami height was reported to be $13 \mathrm{~m}$, while the 1960 tsunami height was $1 \mathrm{~m}$.

At Omoto in Iwaizumi Town, the 1896, 1933, and 2011 tsunamis had similar inundation areas (Fig. 16). The 1960 Chilean tsunami reportedly inundated about $1 \mathrm{~km}$ from the river mouth and raised the water level by $1 \mathrm{~m}$ (CFI 1961). The 2011 tsunami topped the coastal levee and floodgate, which was constructed in 1990. The measured inundation height was $5 \mathrm{~m}$ in the residential area east of the Omoto River (I48). West of the Omoto River, the tsunami inundated farmland and the tsunami heights ranged from 6 to $10 \mathrm{~m}$ (I43I46). Seaward of the Omoto floodgate, the measured runup height was $20 \mathrm{~m}$ (I47). The 1896 tsunami heights were between 10 and $20 \mathrm{~m}$. The reported 1933 tsunami heights were between 3 and $13 \mathrm{~m}$. The 1960 tsunami heights were reported as $4 \mathrm{~m}$ near I47, 3-4 $\mathrm{m}$ at the river mouth by CFI (1961), and $1 \mathrm{~m}$ by JMA (1961).

At Taro in Miyako City, the 2011 tsunami was larger than the 1933 tsunami, but it is unclear whether it was larger than the 1896 tsunami. Tsunami heights from the 2011 event ranged from 12 to 19 m (I69I73). The 1896 heights were reported as being 110 shaku (33 m; YAMANA 1896) or 48 feet $(15 \mathrm{~m}$; IKI 1897), while the heights from the 1933 tsunami ranged from 4 to $10 \mathrm{~m}$. The damage to an icehouse of the Japan Fisheries Cooperatives indicates that the 
Table 1

Tsunami heights for Sanriku coasts of Aomori, Iwate, and Miyagi prefectures from the 1896, 1933, and 2011 earthquakes

\begin{tabular}{|c|c|c|c|c|c|c|c|c|c|c|c|c|c|c|c|}
\hline \multirow[t]{2}{*}{ No. } & \multirow{2}{*}{$\begin{array}{l}\text { Location } \\
\text { Name }\end{array}$} & \multicolumn{2}{|c|}{ Latitude } & \multicolumn{2}{|c|}{ Longitude } & \multirow{2}{*}{$\begin{array}{c}2011 \text { height }^{\mathrm{a}} \\
(\mathrm{m})\end{array}$} & \multirow[t]{2}{*}{ rel. $^{\mathrm{b}}$} & \multirow[t]{2}{*}{ acc. $^{c}$} & \multirow[t]{2}{*}{ type } & \multicolumn{3}{|c|}{1896 height $^{d}$} & \multicolumn{3}{|c|}{1933 height $^{\mathrm{e}}$} \\
\hline & & deg & $\min$ & deg & min. & & & & & $\begin{array}{c}\text { Iki } \\
\text { (feet) }\end{array}$ & $\begin{array}{c}\text { Yaman } \\
\text { a }\end{array}$ & $\begin{array}{c}\text { Matsuo } \\
(\mathrm{m})\end{array}$ & $\begin{array}{l}\text { ERI } \\
(\mathrm{m})\end{array}$ & $\begin{array}{c}\text { Matsuo } \\
(\mathrm{m})\end{array}$ & $\begin{array}{c}\text { Kunitomi } \\
(\mathrm{m})\end{array}$ \\
\hline A3 & $\begin{array}{l}\text { Daisakutai, Samemachi, Hachinohe City } \\
\text { (Okuki Fishing Port) }\end{array}$ & 40 & 30 & 141 & 38 & 9.9 & A & $\mathrm{a}$ & I & & & & & 3.7 & \\
\hline A4 & $\begin{array}{l}\text { Oja, Dobutsu, Hashikami Town (Oja Fishing } \\
\text { Port) }\end{array}$ & 40 & 28 & 141 & 39 & 9.3 & A & $\mathrm{a}$ & $\mathrm{R}$ & & & & 6.0 & 6.0 & 6.0 \\
\hline A5 & $\begin{array}{l}\text { Kominato, Dobutsu, Hashikami Town } \\
\text { (Kominato Fishing Port) }\end{array}$ & 40 & 27 & 141 & 40 & 6.0 & $\mathrm{~A}$ & $\mathrm{a}$ & $\mathrm{R}$ & 20 & & & & 4.5 & 4.5 \\
\hline $\mathrm{I} 2$ & Taneichi, Hirono Town (Taneichi Fishing Port) & 40 & 24 & 141 & 43 & 6.8 & $\mathrm{~B}$ & a & I & 30 & & & 4.5 & & 6.0 \\
\hline $\mathrm{I} 3$ & Taneichi, Hirono Town (Yagi Port) & 40 & 21 & 141 & 46 & 7.8 & B & $\mathrm{b}$ & I & $\Delta 35$ & 20 & $\begin{array}{l}10.5 \\
18.3\end{array}$ & 3.5 & $\begin{array}{l}2.5 \\
4.5 \\
5.3 \\
5.9 \\
7.2\end{array}$ & 6.0 \\
\hline I4 & Uge, Hirono Town (Uge Fishing Port) & 40 & 19 & 141 & 47 & 8.1 & A & a & I & & 28 & & & & \\
\hline I5 & $\begin{array}{c}\text { Nakano, Hirono Town (Koge Fishing Port, Koge } \\
\text { District) }\end{array}$ & 40 & 18 & 141 & 48 & 14.8 & B & $\mathrm{a}$ & $R$ & & & & & & 7.0 \\
\hline I7 & \begin{tabular}{|c|} 
Samuraihamacho-Mukaicho, Kuji City \\
(Kawatsunai Fishing Port, Kawatsunai District)
\end{tabular} & 40 & 16 & 141 & 49 & 12.9 & B & $\mathrm{a}$ & $\mathrm{R}$ & & & & & & 10.6 \\
\hline I8 & $\begin{array}{c}\text { Samuraihamacho-Shiromae, Kuji City } \\
\text { (Shiromae Fishing Port, Shiromae District) }\end{array}$ & 40 & 15 & 141 & 49 & 15.3 & A & $\mathrm{a}$ & $\mathrm{R}$ & 62 & 40 & & & & \\
\hline I9 & $\begin{array}{c}\text { Samuraihamacho-Honnami, Kuji City (Shiromae } \\
\text { Fishing Port, Honnami District) }\end{array}$ & 40 & 14 & 141 & 50 & 19.8 & B & $\mathrm{a}$ & $\bar{R}$ & & 65 & & & & \\
\hline I10 & $\begin{array}{c}\text { Samuraihamacho-Mugyo, Kuji City (Mugyo } \\
\text { Fishing Port) }\end{array}$ & 40 & 13 & 141 & 50 & 18.9 & B & $\mathrm{a}$ & $\mathrm{R}$ & 28 & 50 & & & & \\
\hline I11 & $\begin{array}{l}\text { Osanaicho (Tamanowaki), Kuji City } \\
\text { (Tamanowaki Fishing Port) }\end{array}$ & 40 & 11 & 141 & 48 & 10.7 & $\mathrm{~A}$ & $\mathrm{a}$ & $\bar{R}$ & & 30 & 11.0 & $\begin{array}{l}3.3 \\
4.0\end{array}$ & $\begin{array}{l}6.5 \\
7.9 \\
8.7 \\
\end{array}$ & 5.5 \\
\hline I12 & $\begin{array}{c}\text { Ubecho (Kosode), Kuji City (Kosode Fishing } \\
\text { Port, Kosode District) }\end{array}$ & 40 & 10 & 141 & 51 & 12.0 & B & $\mathrm{a}$ & I & 45 & 180 & $\begin{array}{l}20.3 \\
20.3\end{array}$ & & $\begin{array}{l}10.0 \\
13.3\end{array}$ & 8.2 \\
\hline I13 & Ubecho (Kosode), Kuji City & 40 & 10 & 141 & 51 & 8.2 & $\mathrm{~A}$ & $\mathrm{a}$ & $\mathrm{R}$ & & & & & & \\
\hline I15 & Ubecho (Kuki), Kuji City (Kuki Fishing Port) & 40 & 8 & 141 & 51 & 14.6 & A & $\mathrm{b}$ & I & 40 & 70 & & 3.3 & & 5.5 \\
\hline I16 & Noda, Noda Village (Noda Fishing Port) & 40 & 7 & 141 & 50 & 22.8 & $\mathrm{C}$ & $\mathrm{a}$ & $\mathrm{R}$ & & & 18.3 & 5.5 & 6.4 & 5.5 \\
\hline $\mathrm{I} 17$ & & 40 & 7 & 141 & 50 & 16.4 & $\mathrm{~B}$ & $\mathrm{a}$ & $\mathrm{R}$ & & & 20.0 & & 14.0 & \\
\hline $\mathrm{I} 18$ & & 40 & 7 & 141 & 50 & 18.5 & $\mathrm{~B}$ & $\mathrm{a}$ & $\mathrm{R}$ & & & & & 15.6 & \\
\hline $\mathrm{I} 19$ & Noda (Maita), Noda Village & 40 & 6 & 141 & 50 & 32.0 & $\mathrm{~B}$ & $\mathrm{~b}$ & $\mathrm{R}$ & & & 10.5 & & 6.5 & \\
\hline $\mathrm{I} 20$ & $\begin{array}{c}\text { Tamagawa, Noda Village (Noda Tamagawa } \\
\text { Station) }\end{array}$ & 40 & 5 & 141 & 50 & 26.6 & B & a & $\mathrm{R}$ & 60 & 60 & $\begin{array}{l}20.3 \\
20.3 \\
\end{array}$ & 4.2 & $\begin{array}{l}10.0 \\
13.3\end{array}$ & 5.8 \\
\hline $\mathrm{I} 21$ & Tamagawa (Shimoakka), Noda Village & 40 & 3 & 141 & 51 & 11.8 & B & $\mathrm{a}$ & $\mathrm{R}$ & & 50 & & 5.0 & & \\
\hline $\mathrm{I} 22$ & Otanabe, Fudai Village (Otanabe Fishing Port) & 40 & 1 & 141 & 54 & 8.4 & A & $\mathrm{c}$ & I & 50 & 65 & 17.9 & 7.4 & 12.3 & 13.0 \\
\hline $\mathrm{I} 23$ & & 40 & 1 & 141 & 54 & 8.9 & $\mathrm{~A}$ & $\mathrm{a}$ & $\mathrm{I}$ & & & 30.3 & & 12.6 & \\
\hline $\mathrm{I} 24$ & & 40 & 1 & 141 & 54 & 12.4 & $\mathrm{C}$ & $\mathrm{a}$ & $\mathrm{R}$ & & & & & 18.3 & \\
\hline I25 & & 40 & 1 & 141 & 54 & 10.6 & B & a & $\mathrm{R}$ & & & & & 21.1 & \\
\hline $\mathrm{I} 32$ & Aketo, Tanohata Village & 39 & 57 & 141 & 57 & 19.2 & $\mathrm{~B}$ & $\mathrm{a}$ & $\mathrm{R}$ & $\Delta 40$ & & & 8.4 & & 16.9 \\
\hline $\mathrm{I} 33$ & & 39 & 57 & 141 & 57 & 22.6 & $\mathrm{~B}$ & $\mathrm{a}$ & $\mathrm{R}$ & & & & 8.4 & & \\
\hline $\mathrm{I} 34$ & Raga, Tanohata Village & 39 & 56 & 141 & 56 & 23.9 & $\mathrm{~A}$ & $\mathrm{a}$ & $\mathrm{I}$ & 75 & 60 & 24.3 & & 13.3 & 13.0 \\
\hline $\mathrm{I} 35$ & & 39 & 56 & 141 & 56 & 22.9 & $\mathrm{~A}$ & $\mathrm{a}$ & $\mathrm{R}$ & & & 24.3 & & 13.3 & \\
\hline $\mathrm{I} 36$ & & 39 & 56 & 141 & 56 & 27.8 & $\mathrm{~B}$ & $\mathrm{a}$ & $\mathrm{R}$ & & & 26.3 & & 13.3 & \\
\hline $\mathrm{I} 37$ & & 39 & 56 & 141 & 56 & 24.5 & $\mathrm{~B}$ & $\mathrm{a}$ & $\mathrm{R}$ & & & & & & \\
\hline $\mathrm{I} 38$ & Raga (Hiraiga), Tanohata Village & 39 & 56 & 141 & 56 & 17.5 & B & a & $\mathrm{R}$ & & 40 & 14.3 & 8.2 & 9.3 & 8.2 \\
\hline $\mathrm{I} 39$ & & 39 & 56 & 141 & 56 & 16.5 & B & $\mathrm{a}$ & $\mathrm{R}$ & & & $\begin{array}{l}16.3 \\
18.3\end{array}$ & 8.3 & $\begin{array}{l}10.3 \\
10.3\end{array}$ & \\
\hline I40 & Wano, Tanohata Village & 39 & 56 & 141 & 56 & 19.0 & B & a & I & & & $\begin{array}{l}19.3 \\
20.3 \\
\end{array}$ & & 10.3 & \\
\hline I41 & Shimanokoshi, Tanohata Village & 39 & 55 & 141 & 56 & 19.9 & $\mathrm{~B}$ & $\mathrm{a}$ & $\mathrm{R}$ & & 132 & $\begin{array}{l}14.3 \\
14.3\end{array}$ & $\begin{array}{l}4.5 \\
4.5\end{array}$ & $\begin{array}{l}7.0 \\
7.5\end{array}$ & 9.7 \\
\hline I42 & & 39 & 55 & 141 & 56 & 22.0 & A & $\mathrm{a}$ & $\mathrm{R}$ & & & $\begin{array}{l}15.3 \\
19.9 \\
24.1\end{array}$ & 6.0 & $\begin{array}{r}8.3 \\
8.5 \\
9.8 \\
11.1 \\
\end{array}$ & \\
\hline I43 & Omoto, Iwaizumi Town & 39 & 51 & 141 & 58 & 10.3 & $\mathrm{~B}$ & $\mathrm{a}$ & $\mathrm{R}$ & 40 & 65 & 10.0 & $3.4-4.4$ & 8.5 & \\
\hline I44 & & 39 & 51 & 141 & 58 & 6.5 & $\mathrm{~A}$ & $\mathrm{a}$ & I & & & 11.8 & 5.0 & 9.1 & \\
\hline I45 & & 39 & 51 & 141 & 58 & 5.6 & A & $\mathrm{a}$ & I & & & & & 9.4 & \\
\hline I46 & & 39 & 51 & 141 & 58 & 6.9 & A & $\mathrm{a}$ & I & & & & & $\begin{array}{l}10.1 \\
104\end{array}$ & \\
\hline I47 & & 39 & 51 & 141 & 58 & 20.4 & $\mathrm{~B}$ & a & $\mathrm{R}$ & & & & & 10.6 & 13.0 \\
\hline $\mathrm{I} 48$ & & 39 & 51 & 141 & 58 & 5.4 & A & a & I & & & & & $\begin{array}{l}13.0 \\
13.4\end{array}$ & \\
\hline $\mathrm{I} 49$ & Moshi, Omoto, Iwaizumi Town (Moshi Fishing & 39 & 50 & 141 & 59 & 24.5 & $\mathrm{~B}$ & $\mathrm{a}$ & $\mathrm{R}$ & & & 17.7 & & 13.6 & \\
\hline $\mathrm{I} 50$ & Port) & 39 & 50 & 141 & 59 & 23.5 & $\mathrm{~B}$ & $\mathrm{a}$ & $\mathrm{R}$ & & & 18.8 & & 14.2 & \\
\hline I51 & Moshi, Omoto, Iwaizumi Town & 39 & 50 & 141 & 58 & 22.3 & $\mathrm{~B}$ & $\mathrm{a}$ & $\mathrm{R}$ & & & 24.0 & & 15.1 & \\
\hline $\mathrm{I} 52$ & & 39 & 50 & 141 & 58 & 22.2 & $\mathrm{~B}$ & $\mathrm{a}$ & $\mathrm{R}$ & & & & & 15.1 & \\
\hline
\end{tabular}


Table 1

continued

\begin{tabular}{|c|c|c|c|c|c|c|c|c|c|c|c|c|c|c|c|}
\hline \multirow[t]{2}{*}{ No. } & \multirow{2}{*}{$\begin{array}{c}\text { Location } \\
\text { Name }\end{array}$} & \multicolumn{2}{|c|}{ Latitude } & \multicolumn{2}{|c|}{ Longitude } & \multirow{2}{*}{$\frac{2011 \text { height }^{\mathrm{a}}}{(\mathrm{m})}$} & \multirow[t]{2}{*}{ rel. $^{\mathrm{b}}$} & \multirow[t]{2}{*}{ acc. $^{\mathrm{c}}$} & \multirow[t]{2}{*}{ type } & \multicolumn{3}{|c|}{1896 height $^{d}$} & & 1933 heig & \\
\hline & & deg & $\min$ & $\operatorname{deg}$ & $\min$. & & & & & $\begin{array}{c}\text { Iki } \\
\text { (feet) }\end{array}$ & \begin{tabular}{c|} 
Yaman \\
a
\end{tabular} & $\begin{array}{c}\text { Matsuo } \\
(\mathrm{m})\end{array}$ & $\begin{array}{l}\text { ERI } \\
(\mathrm{m})\end{array}$ & $\begin{array}{c}\text { Matsuo } \\
(\mathrm{m})\end{array}$ & $\begin{array}{c}\text { Kunitomi } \\
(\mathrm{m})\end{array}$ \\
\hline I53 & Taro-Shimosettai, Miyako City & 39 & 49 & 141 & 59 & 21.8 & $\mathrm{~B}$ & $\mathrm{a}$ & $\mathrm{R}$ & & 45 & & 11.8 & & \\
\hline I54 & & 39 & 49 & 141 & 59 & 27.9 & B & a & $\mathrm{R}$ & & & & & & \\
\hline I55 & & 39 & 49 & 141 & 59 & 22.3 & A & $\mathrm{a}$ & I & & & & & & \\
\hline $\mathrm{I} 60$ & $\begin{array}{c}\text { Taro-Aonotakiminami, Miyako City (Aonotaki } \\
\text { Fishing Port) }\end{array}$ & 39 & 46 & 141 & 59 & 34.7 & $\mathrm{~B}$ & $\mathrm{~b}$ & $\mathrm{R}$ & & & & 10.3 & & \\
\hline I61 & Taro-Otobeno, Miyako City & 39 & 45 & 141 & 59 & 22.9 & $\mathrm{~B}$ & $\mathrm{a}$ & $\mathrm{R}$ & & 110 & & 7.2 & & \\
\hline I62 & & 39 & 45 & 141 & 59 & 16.5 & $\mathrm{~B}$ & $\mathrm{a}$ & $\mathrm{R}$ & & & & 10 & & \\
\hline I63 & & 39 & 45 & 141 & 59 & 23.3 & $\mathrm{~B}$ & $\mathrm{a}$ & $\mathrm{R}$ & & & & 10.2 & & \\
\hline I64 & & 39 & 45 & 141 & 59 & 30.1 & $\mathrm{~B}$ & $\mathrm{a}$ & $\mathrm{R}$ & & & & & & \\
\hline I65 & & 39 & 45 & 141 & 59 & 34.1 & $\mathrm{~B}$ & $\mathrm{a}$ & $\mathrm{R}$ & & & & & & \\
\hline I69 & Taro-Tategamori, Miyako City & 39 & 44 & 141 & 58 & 12.6 & $\mathrm{~A}$ & $\mathrm{a}$ & $\mathrm{I}$ & 48 & 110 & & 4 & 6.4 & \\
\hline $\mathrm{I} 70$ & Taro-Nohara, Miyako City & 39 & 44 & 141 & 59 & 14.8 & $\mathrm{~A}$ & $\mathrm{c}$ & $\mathrm{I}$ & & & & 7 & & \\
\hline I71 & Taro-Aozari, Miyako City & 39 & 44 & 141 & 59 & 19.0 & $\mathrm{~B}$ & $\mathrm{~b}$ & $\mathrm{R}$ & & & & 10 & & \\
\hline $\mathrm{I} 72$ & & 39 & 44 & 141 & 59 & 14.8 & A & $\mathrm{c}$ & $\mathrm{I}$ & & & & & & \\
\hline I73 & Taro-Tanaka, Miyako City & 39 & 44 & 141 & 58 & 11.8 & $\mathrm{~B}$ & $\mathrm{a}$ & $\mathrm{R}$ & & & & & & 10.1 \\
\hline 174 & Taro-Nishimukaiyama, Miyako City (Kashinai & 39 & 43 & 141 & 58 & 25.5 & $\mathrm{~B}$ & $\mathrm{a}$ & $\mathrm{R}$ & & & & 5 & & \\
\hline $\mathrm{I} 75$ & & 39 & 42 & 141 & 59 & 17.5 & $\mathrm{~B}$ & a & $\mathrm{R}$ & & & & & & \\
\hline I76 & $\begin{array}{c}\text { Taro-Kashinai, Miyako City (Kashinai Fishing } \\
\text { Port) }\end{array}$ & 39 & 42 & 141 & 58 & 21.8 & $\mathrm{~B}$ & $\mathrm{a}$ & $\mathrm{R}$ & & & & & & \\
\hline $\mathrm{I} 77$ & Sakiyama (Mattsuki), Miyako City & 39 & 42 & 141 & 58 & 31.4 & $\mathrm{~B}$ & $\mathrm{a}$ & $\mathrm{R}$ & & & & 4 & & \\
\hline $\mathrm{I} 78$ & & 39 & 42 & 141 & 58 & 28.7 & $\mathrm{~B}$ & $\mathrm{a}$ & $\mathrm{R}$ & & & & & & \\
\hline I79 & & 39 & 42 & 141 & 58 & 28.0 & $\mathrm{~B}$ & $\mathrm{a}$ & $\mathrm{R}$ & & & & & & \\
\hline $\mathrm{I} 80$ & Sakiyama (Onappe), Miyako City & 39 & 42 & 141 & 58 & 15.1 & $\mathrm{~A}$ & $\mathrm{a}$ & $\mathrm{R}$ & & 40 & & 4.5 & & 7.5 \\
\hline I81 & & 39 & 42 & 141 & 58 & 14.8 & $\mathrm{~B}$ & $\mathrm{a}$ & $\mathrm{R}$ & & & & & & \\
\hline I82 & & 39 & 41 & 141 & 58 & 12.6 & $\mathrm{~B}$ & $\mathrm{a}$ & $\mathrm{I}$ & & & & & & \\
\hline I83 & & 39 & 41 & 141 & 58 & 17.6 & $\mathrm{~B}$ & $\mathrm{a}$ & $\mathrm{R}$ & & & & & & \\
\hline I 84 & & 39 & 41 & 141 & 58 & 20.6 & $\mathrm{~B}$ & $\mathrm{a}$ & $\mathrm{R}$ & & & & & & \\
\hline $\mathrm{I} 85$ & & 39 & 41 & 141 & 58 & 26.0 & $\mathrm{~B}$ & $\mathrm{a}$ & $\mathrm{R}$ & & & & & & \\
\hline $\mathrm{I} 86$ & Kuwagasaki (Nakamachi), Miyako City & 39 & 39 & 141 & 58 & 8.2 & A & $\mathrm{a}$ & I & $\Delta 30$ & 20 & & $\begin{array}{l}3 \\
4\end{array}$ & $\begin{array}{l}3.7 \\
5.5\end{array}$ & \\
\hline I 88 & Tsugaruishi (Norinowaki), Miyako City & 39 & 35 & 141 & 57 & 11.6 & B & a & $\mathrm{R}$ & & 12 & & 1.5 & & 1.6 \\
\hline $\mathrm{I} 89$ & Akamae, Miyako City & 39 & 36 & 141 & 58 & 9.5 & $\mathrm{~B}$ & $\mathrm{a}$ & $\mathrm{R}$ & & 15 & & & & 1.7 \\
\hline $\mathrm{I} 90$ & & 39 & 36 & 141 & 58 & 14.0 & $\mathrm{~B}$ & $\mathrm{a}$ & $\mathrm{R}$ & & & & & & \\
\hline I91 & Omoe (Tatehama), Miyako City & 39 & 38 & 142 & 1 & 22.5 & $\mathrm{~B}$ & $\mathrm{a}$ & $\mathrm{R}$ & & 60 & & 8.2 & & \\
\hline $\mathrm{I} 92$ & & 39 & 38 & 142 & 1 & 25.2 & $\mathrm{~B}$ & $\mathrm{a}$ & $\mathrm{R}$ & & & & 12 & & \\
\hline $\mathrm{I} 93$ & & 39 & 38 & 142 & 1 & 26.1 & $\mathrm{~B}$ & $\mathrm{a}$ & $\mathrm{R}$ & & & & & & \\
\hline I94 & Omoe (Shukuhama), Miyako City & 39 & 38 & 142 & 1 & 25.1 & $\mathrm{~B}$ & $\mathrm{a}$ & $\mathrm{R}$ & & 70 & & $9.6-$ & & \\
\hline $\mathrm{I} 95$ & & 39 & 38 & 142 & 1 & 24.6 & $\mathrm{~B}$ & $\mathrm{a}$ & $\mathrm{R}$ & & & & 11.5 & & \\
\hline I96 & Omoe (Uiso), Miyako City & 39 & 37 & 142 & 1 & 27.0 & $\mathrm{~B}$ & $\mathrm{a}$ & $\mathrm{R}$ & & 70 & & 9.7 & & 4.5 \\
\hline $\mathrm{I} 97$ & & 39 & 37 & 142 & 1 & 25.4 & $\mathrm{~A}$ & $\mathrm{a}$ & $\mathrm{I}$ & & & & 12.8 & & \\
\hline $\mathrm{I} 98$ & & 39 & 37 & 142 & 1 & 23.2 & $\mathrm{~B}$ & $\mathrm{a}$ & $\mathrm{R}$ & & & & 13 & & \\
\hline $\mathrm{I} 99$ & & 39 & 37 & 142 & 1 & 24.5 & $\mathrm{~A}$ & $\mathrm{a}$ & $\mathrm{I}$ & & & & & & \\
\hline $\mathrm{I} 100$ & Omoe (Aramaki), Miyako City & 39 & 36 & 142 & 1 & 21.1 & $\mathrm{~B}$ & $\mathrm{a}$ & $\mathrm{R}$ & $\Delta 40$ & 80 & & 7.2 & & 7.6 \\
\hline I101 & & 39 & 36 & 142 & 1 & 27.7 & $\mathrm{~B}$ & $\mathrm{a}$ & $\mathrm{R}$ & & & & 8.7 & & \\
\hline $\mathrm{I} 102$ & & 39 & 36 & 142 & 1 & 23.5 & $\mathrm{~B}$ & a & $\mathrm{R}$ & & & & & & \\
\hline I103 & Omoe (Sato), Miyako City (Omoe Fishing Port) & 39 & 34 & 142 & 2 & 20.1 & B & a & $\mathrm{R}$ & & 40 & & 8.3 & & 10.9 \\
\hline I104 & & 39 & 34 & 142 & 2 & 34.1 & $\mathrm{C}$ & a & $\mathrm{R}$ & & & & $\begin{array}{c}8.8 \\
14\end{array}$ & & \\
\hline $\mathrm{I} 105$ & Omoe (Aneyoshi), Miyako City & 39 & 32 & 142 & 3 & 26.5 & $\mathrm{~B}$ & $\mathrm{a}$ & $\mathrm{R}$ & 62 & 110 & & 12.8 & 14.0 & 12.4 \\
\hline $\mathrm{I} 106$ & & 39 & 32 & 142 & 3 & 22.8 & $\mathrm{~B}$ & $\mathrm{a}$ & $\mathrm{R}$ & & & & 14.0 & & \\
\hline I107 & & 39 & 32 & 142 & 3 & 24.5 & B & $\mathrm{c}$ & $\mathrm{R}$ & & & & $\begin{array}{c}20 \\
21.0\end{array}$ & & \\
\hline I108 & Omoe (Chikei), Miyako City & 39 & 32 & 142 & 2 & 29.5 & $\mathrm{~A}$ & $\mathrm{a}$ & $\mathrm{R}$ & 56 & 80 & & 10.9 & & 6.0 \\
\hline I109 & & 39 & 32 & 142 & 2 & 31.2 & $\mathrm{~A}$ & $\mathrm{a}$ & $\mathrm{R}$ & & & & 11.0 & & 13.6 \\
\hline I110 & & 39 & 32 & 142 & 2 & 29.4 & A & $\mathrm{a}$ & $\mathrm{R}$ & & & & $\begin{array}{l}12.1 \\
13.2\end{array}$ & & \\
\hline I111 & Omoe (Ishihama), Miyako City & 39 & 31 & 142 & 2 & 20.0 & $\mathrm{~A}$ & $\mathrm{a}$ & I & & 30 & & $\begin{array}{l}7.2 \\
8.7 \\
\end{array}$ & & 12.0 \\
\hline $\mathrm{I} 112$ & Funakoshi (Ohura), Yamada Town & 39 & 27 & 142 & 0 & 8.0 & $\mathrm{~B}$ & $\mathrm{a}$ & $\mathrm{R}$ & 16 & 20 & & & & \\
\hline I113 & & 39 & 27 & 142 & 0 & 7.7 & $\mathrm{~A}$ & $\mathrm{a}$ & $\mathrm{I}$ & & & & & & \\
\hline $\mathrm{I} 114$ & & 39 & 27 & 142 & 0 & 8.0 & $\mathrm{~B}$ & $\mathrm{a}$ & $\mathrm{R}$ & & & & & & \\
\hline I115 & & 39 & 27 & 142 & 0 & 7.8 & $\mathrm{~B}$ & $\mathrm{a}$ & $\mathrm{R}$ & & & & & & \\
\hline I116 & Funakoshi (Sukuiso), Yamada Town & 39 & 27 & 142 & 3 & 29.7 & $\mathrm{~B}$ & $\mathrm{a}$ & $\mathrm{R}$ & 50 & 40 & & & & \\
\hline I117 & & 39 & 27 & 142 & 3 & 28.2 & $\mathrm{~B}$ & $\mathrm{a}$ & $\mathrm{R}$ & & & & & & \\
\hline I118 & Funakoshi (Koyadori), Yamada Town & 39 & 26 & 142 & 1 & 26.1 & A & $\mathrm{a}$ & $\mathrm{R}$ & 50 & 50 & & 6.6 & & \\
\hline I119 & & 39 & 26 & 142 & 1 & 25.0 & $\mathrm{~B}$ & $\mathrm{a}$ & $\mathrm{R}$ & & & & & & \\
\hline $\mathrm{I} 120$ & Akahama, Otsuchi Town & 39 & 21 & 141 & 56 & 13.3 & $\mathrm{~B}$ & $\mathrm{a}$ & $\mathrm{R}$ & & 20 & & & & 4.6 \\
\hline $\mathrm{I} 121$ & Akahama, Otsuchi Town & 39 & 21 & 141 & 56 & 11.5 & $\mathrm{~A}$ & $\mathrm{a}$ & $\mathrm{I}$ & & & & & & \\
\hline $\mathrm{I} 122$ & $\begin{array}{c}\text { (International Coastal Research Center, } \\
\text { Atmosphere and Ocean Research Institute, the } \\
\text { University of Tokyo) }\end{array}$ & 39 & 21 & 141 & 56 & 12.1 & $\mathrm{~A}$ & $\mathrm{a}$ & $\mathrm{I}$ & & & & & & \\
\hline
\end{tabular}


Table 1

continued

\begin{tabular}{|c|c|c|c|c|c|c|c|c|c|c|c|c|c|c|c|}
\hline \multirow[t]{2}{*}{ No. } & \multirow{2}{*}{$\begin{array}{c}\text { Location } \\
\text { Name }\end{array}$} & \multicolumn{2}{|c|}{ Latitude } & \multicolumn{2}{|c|}{ Longitude } & \multirow{2}{*}{\begin{tabular}{|c|}
2011 height $^{\mathrm{a}}$ \\
$(\mathrm{m})$
\end{tabular}} & \multirow[t]{2}{*}{ rel. $^{\mathrm{b}}$} & \multirow[t]{2}{*}{ acc. $^{\mathrm{c}}$} & \multirow[t]{2}{*}{ type } & \multicolumn{3}{|c|}{1896 height $^{\mathrm{d}}$} & \multicolumn{3}{|c|}{1933 height $^{\mathrm{e}}$} \\
\hline & & deg & $\min$ & deg & $\min$. & & & & & $\begin{array}{c}\text { Iki } \\
\text { (feet) }\end{array}$ & $\begin{array}{c}\text { Yaman } \\
\text { a }\end{array}$ & $\begin{array}{c}\text { Matsuo } \\
(\mathrm{m})\end{array}$ & $\begin{array}{l}\text { ERI } \\
(\mathrm{m})\end{array}$ & $\begin{array}{c}\text { Matsuo } \\
(\mathrm{m})\end{array}$ & $\begin{array}{c}\text { Kunitomi } \\
(\mathrm{m})\end{array}$ \\
\hline $\mathrm{I} 123$ & Omachi, Kamaishi City & 39 & 16 & 141 & 53 & 5.4 & $\mathrm{~A}$ & $\mathrm{~b}$ & $\mathrm{I}$ & $15-27$ & 50 & $\begin{array}{l}6.0 \\
6.0 \\
7.2\end{array}$ & $\begin{array}{l}3.0 \\
3.5 \\
3.7 \\
3.8 \\
3.9\end{array}$ & $\begin{array}{l}2.7 \\
3.6 \\
3.9 \\
4.2 \\
4.2 \\
4.4 \\
4.4\end{array}$ & 5.4 \\
\hline $\mathrm{I} 124$ & \multirow{3}{*}{$\begin{array}{c}\text { Osone, Tonicho, } \\
\text { Kamaishi City }\end{array}$} & 39 & 13 & 141 & 53 & 20.3 & $\mathrm{~A}$ & a & $\mathrm{R}$ & & & 15.3 & \multirow{5}{*}{$\begin{array}{l}11.1 \\
11.5 \\
11.8 \\
12.0\end{array}$} & \multirow{5}{*}{$\begin{array}{l}9.9 \\
9.9\end{array}$} & \multirow[t]{5}{*}{6.0} \\
\hline I125 & & 39 & 13 & 141 & 53 & 16.7 & $\mathrm{C}$ & $\mathrm{a}$ & I & & & 15.3 & & & \\
\hline $\mathrm{I} 126$ & & 39 & 13 & 141 & 53 & 16.8 & $\mathrm{~B}$ & $\mathrm{a}$ & $\mathrm{R}$ & & & & & & \\
\hline I127 & Sakuratoge, Tonicho, & 39 & 12 & 141 & 53 & 13.7 & $\mathrm{~B}$ & $\mathrm{a}$ & $\mathrm{R}$ & & & & & & \\
\hline I128 & Kamaishi City & 39 & 12 & 141 & 53 & 12.7 & B & $\mathrm{a}$ & $\mathrm{R}$ & & & & & & \\
\hline I129 & Arakawa, Tonicho, Kamaishi City & 39 & 12 & 141 & 52 & 16.5 & $\mathrm{~B}$ & $\mathrm{a}$ & $\mathrm{R}$ & $\Delta 35$ & 30 & $\begin{array}{l}13.3 \\
13.3 \\
\end{array}$ & 5.8 & $\begin{array}{l}8.1 \\
8.1\end{array}$ & \\
\hline $\mathrm{I} 130$ & \multirow[t]{3}{*}{ Horei, Sanrikucho-Okirai, Ofunato City } & 39 & 6 & 141 & 48 & 17.8 & $\mathrm{~B}$ & $\mathrm{a}$ & $\mathrm{R}$ & & \multirow[t]{3}{*}{50} & & \multirow{3}{*}{$\begin{array}{c}12 \\
10.2\end{array}$} & \multirow[t]{3}{*}{8.3} & \multirow[t]{3}{*}{4.2} \\
\hline $\mathrm{I} 131$ & & 39 & 6 & 141 & 48 & 14.8 & $\mathrm{~B}$ & $\mathrm{a}$ & $\mathrm{R}$ & & & & & & \\
\hline $\mathrm{I} 132$ & & 39 & 6 & 141 & 48 & 15.2 & $\mathrm{~B}$ & $\mathrm{a}$ & $\mathrm{R}$ & & & & & & \\
\hline I133 & Shirahama, Sanrikucho-Ryori, Ofunato City & 39 & 4 & 141 & 49 & 16.8 & B & a & $\mathrm{R}$ & \multirow[t]{2}{*}{72} & $\begin{array}{c}130- \\
180\end{array}$ & 38.2 & 13 & $\begin{array}{l}23.3 \\
23.3\end{array}$ & 23.0 \\
\hline I134 & Okubo, Sanrikucho-Ryori, Ofunato City & 39 & 3 & 141 & 49 & 21.2 & $\mathrm{~B}$ & $\mathrm{a}$ & $\mathrm{R}$ & & & & $\begin{array}{l}18.4 \\
20.7 \\
22.1 \\
23.0 \\
28.7\end{array}$ & $\begin{array}{l}24.3 \\
29.2\end{array}$ & \\
\hline I135 & Miyanomae, Ofunatocho, Ofunato City & 39 & 2 & 141 & 43 & 7.6 & A & a & I & & & & $\begin{array}{l}3.2 \\
3.7 \\
4.1 \\
\end{array}$ & & 3.0 \\
\hline I136 & $\begin{array}{c}\text { Yonesakicho, } \\
\text { Rikuzentakata City }\end{array}$ & 39 & 1 & 141 & 40 & 18.1 & $\mathrm{~B}$ & $\mathrm{~b}$ & $\mathrm{R}$ & & & & & & 3.2 \\
\hline M1 & Karakuwacho-Baba, Kesennuma City & 38 & 54 & 141 & 39 & 13.1 & A & $\mathrm{a}$ & $\mathrm{R}$ & 32 & & $\begin{array}{c}9.9 \\
10.9 \\
11.6\end{array}$ & $\begin{array}{c}7 \\
8.1\end{array}$ & & 5.6 \\
\hline M3 & Karakuwacho-Kakehama, Kesennuma City & 38 & 53 & 141 & 40 & 15.6 & A & $\mathrm{a}$ & I & & & $\begin{array}{c}8.4 \\
10.8 \\
11.2 \\
\end{array}$ & $\begin{array}{c}13 \\
15 \\
20.5 \\
\end{array}$ & & 12.6 \\
\hline M4 & Karakuwacho-Tsumoto, Kesennuma City & 38 & 52 & 141 & 40 & 10.1 & A & $\mathrm{a}$ & $\mathrm{I}$ & & & & 7.0 & & \\
\hline M5 & Minamimachikaigan, Kesennuma City & 38 & 54 & 141 & 34 & 4.0 & $\mathrm{~A}$ & $\mathrm{a}$ & $\mathrm{I}$ & & & & & & 1.0 \\
\hline M6 & (Kesennuma Port) & 38 & 54 & 141 & 34 & 3.7 & $\mathrm{~A}$ & $\mathrm{a}$ & $\mathrm{I}$ & & & & & & \\
\hline M7 & & 38 & 54 & 141 & 34 & 4.1 & $\mathrm{~A}$ & $\mathrm{a}$ & $\mathrm{I}$ & & & & & & \\
\hline M8 & & 38 & 54 & 141 & 35 & 4.1 & $\mathrm{~A}$ & $\mathrm{a}$ & I & & & & & & \\
\hline M9 & Hajikamisuginoshita, Kesennuma City & 38 & 50 & 141 & 35 & 13.7 & A & $\mathrm{b}$ & I & & & $\begin{array}{l}4.0 \\
4.9 \\
6.1 \\
7.3\end{array}$ & $\begin{array}{l}2.9 \\
4.5 \\
4.6 \\
7.6 \\
7.8 \\
\end{array}$ & $\begin{array}{l}2.9 \\
3.2 \\
3.8\end{array}$ & \\
\hline M10 & $\begin{array}{l}\text { Motoyoshicho-Amagasawa, Kesennuma City } \\
\text { (Hikado Fishing Port) }\end{array}$ & 38 & 48 & 141 & 33 & 16.6 & A & $\mathrm{a}$ & $\mathrm{R}$ & 17 & & & $\begin{array}{l}3.3 \\
4.0 \\
4.0 \\
\end{array}$ & 3.3 & 3.0 \\
\hline M11 & Motoyoshicho-Maehama, Kesennuma City & 38 & 48 & 141 & 33 & 19.3 & $\mathrm{~B}$ & a & I & & & $\begin{array}{l}9.3 \\
9.5 \\
\end{array}$ & 5.8 & & \\
\hline M12 & Motoyoshicho-Toyomazawa, Kesennuma City & 38 & 47 & 141 & 31 & 19.7 & $\mathrm{~A}$ & $\mathrm{a}$ & I & & & $\begin{array}{l}5.6 \\
9.4 \\
\end{array}$ & $7-8$ & & \\
\hline M13 & Motoyoshicho-Nijuichihama, Kesennuma City & 38 & 46 & 141 & 31 & 17.4 & A & $\mathrm{a}$ & I & 20 & & 6.3 & 5.9 & 6.2 & \\
\hline M14 & & 38 & 45 & 141 & 31 & 20.9 & B & a & $\mathrm{R}$ & & & $\begin{array}{l}7.7 \\
8.4\end{array}$ & & & \\
\hline M15 & Utatsu-Namiita, Minamisanriku Town & 38 & 45 & 141 & 32 & 19.4 & $\mathrm{~A}$ & $\mathrm{a}$ & $\mathrm{R}$ & 22 & & 5.4 & 3.5 & 3.2 & \\
\hline M16 & & 38 & 45 & 141 & 32 & 18.0 & $\mathrm{~A}$ & $\mathrm{a}$ & $\mathrm{I}$ & & & 6.7 & & & \\
\hline M17 & & 38 & 45 & 141 & 32 & 19.5 & $\mathrm{~A}$ & $\mathrm{a}$ & $\mathrm{R}$ & & & 7.0 & & & \\
\hline M18 & Utatsu-Minato, Minamisanriku Town & 38 & 44 & 141 & 32 & 14.5 & $\mathrm{~A}$ & $\mathrm{a}$ & $\mathrm{R}$ & & & & & & \\
\hline M19 & & 38 & 44 & 141 & 32 & 14.0 & $\mathrm{~A}$ & $\mathrm{a}$ & $\mathrm{R}$ & & & & & & \\
\hline M20 & & 38 & 44 & 141 & 32 & 14.6 & $\mathrm{~A}$ & $\mathrm{a}$ & $\mathrm{I}$ & & & & & & \\
\hline M21 & Utatsu-Tanoura, Minamisanriku Town & 38 & 44 & 141 & 33 & 13.1 & $\mathrm{~B}$ & $\mathrm{a}$ & $\mathrm{R}$ & 16 & & $\begin{array}{l}3.7 \\
8.5\end{array}$ & $\begin{array}{l}4.5 \\
5.1\end{array}$ & $\begin{array}{l}5.0 \\
5.5\end{array}$ & \\
\hline M22 & Utatsu-Kaminoyama, Minamisanriku Town & 38 & 44 & 141 & 33 & 14.3 & $\mathrm{~A}$ & $\mathrm{a}$ & $\mathrm{R}$ & & & 10.7 & & & \\
\hline M23 & & 38 & 44 & 141 & 33 & 16.4 & $\mathrm{~B}$ & $\mathrm{a}$ & $\mathrm{R}$ & & & & & & \\
\hline M25 & Utatsu-Ishihama, Minamisanriku Town & 38 & 44 & 141 & 34 & 15.6 & $\mathrm{~B}$ & $\mathrm{a}$ & I & & & $\begin{array}{c}9.3 \\
12.9 \\
13.0 \\
\end{array}$ & 10.5 & & 7.6 \\
\hline M27 & Utatsu-Tatehama, Minamisanriku Town & 38 & 43 & 141 & 33 & 12.7 & A & $\mathrm{a}$ & I & & & $\begin{array}{l}3.9 \\
4.4 \\
4.7 \\
5.3 \\
\end{array}$ & $\begin{array}{l}3.9 \\
5.2\end{array}$ & $\begin{array}{l}3.3 \\
3.6 \\
3.7 \\
3.8 \\
\end{array}$ & \\
\hline
\end{tabular}


Table 1

continued

\begin{tabular}{|c|c|c|c|c|c|c|c|c|c|c|c|c|c|c|c|}
\hline \multirow[t]{2}{*}{ No. } & \multirow{2}{*}{$\begin{array}{l}\text { Location } \\
\text { Name }\end{array}$} & \multicolumn{2}{|c|}{ Latitude } & \multicolumn{2}{|c|}{ Longitude } & \multirow{2}{*}{$\frac{2011 \text { height }^{\mathrm{a}}}{\mathrm{(m)}}$} & \multirow[t]{2}{*}{ rel. $^{\mathrm{b}}$} & \multirow[t]{2}{*}{ acc. $^{\text {c }}$} & \multirow[t]{2}{*}{ type } & \multicolumn{3}{|c|}{1896 height $^{d}$} & \multicolumn{3}{|c|}{1933 height $^{\mathrm{e}}$} \\
\hline & & deg & $\min$ & deg & $\min$. & & & & & $\begin{array}{c}\text { Iki } \\
\text { (feet) }\end{array}$ & $\begin{array}{c}\text { Yaman } \\
\text { a }\end{array}$ & $\begin{array}{c}\text { Matsuo } \\
(\mathrm{m})\end{array}$ & $\begin{array}{l}\text { ERI } \\
(\mathrm{m})\end{array}$ & $\begin{array}{c}\text { Matsuo } \\
(\mathrm{m})\end{array}$ & $\begin{array}{c}\text { Kunitomi } \\
(\mathrm{m})\end{array}$ \\
\hline M28 & Utatsu-Niranohama, Minamisanriku Town & 38 & 42 & 141 & 30 & 12.9 & A & $\mathrm{a}$ & I & & & $\begin{array}{l}3.5 \\
3.6 \\
3.7 \\
\end{array}$ & 2.9 & $\begin{array}{l}1.6 \\
2.4 \\
2.5 \\
\end{array}$ & \\
\hline M29 & \multirow{2}{*}{ Shizugawa-Nishida, Minamisanriku Town } & 38 & 42 & 141 & 30 & 15.1 & B & $\mathrm{a}$ & $\mathrm{R}$ & \multirow[t]{4}{*}{12} & \multirow{4}{*}{\multicolumn{2}{|c|}{$\begin{array}{l}3.8 \\
4.7 \\
4.7\end{array}$}} & \multirow{4}{*}{2.5} & 1.9 & \multirow[t]{4}{*}{3.6} \\
\hline M30 & & 38 & 42 & 141 & 30 & 15.0 & B & $\mathrm{a}$ & $\mathrm{R}$ & & & & & 1.9 & \\
\hline M31 & \multirow[t]{2}{*}{ Shizugawa-Hosoura, Minamisanriku Town } & 38 & 42 & 141 & 30 & 13.9 & $\mathrm{~B}$ & $\mathrm{a}$ & $\mathrm{R}$ & & & & & 2.2 & \\
\hline M32 & & 38 & 42 & 141 & 30 & 14.5 & $\mathrm{~A}$ & $\mathrm{a}$ & $\mathrm{R}$ & & & & & & \\
\hline M33 & Shizugawa-Omori, Minamisanriku Town & 38 & 41 & 141 & 27 & 14.4 & $\mathrm{C}$ & a & $\mathrm{R}$ & & & & & & 5.4 \\
\hline M34 & Shizugawa-Hayashi, Minamisanriku Town & 38 & 40 & 141 & 27 & 16.0 & $\overline{\mathrm{A}}$ & a & I & 6 & & & 3.2 & & \\
\hline M35 & Mitobe, Tokura, Minamisanriku Town & 38 & 38 & 141 & 27 & 11.7 & $\mathrm{~B}$ & a & $\mathrm{R}$ & $\Delta 8$ & & $\begin{array}{l}2.7 \\
2.9\end{array}$ & $\begin{array}{l}1.9 \\
2.1\end{array}$ & $\begin{array}{l}2.2 \\
2.2 \\
\end{array}$ & \\
\hline M36 & Takihama, Tokura, Minamisanriku Town & 38 & 38 & 141 & 30 & 12.1 & B & $\mathrm{a}$ & I & 13 & & $\begin{array}{l}4.7 \\
6.0\end{array}$ & 2.4 & $\begin{array}{l}2.5 \\
2.5 \\
\end{array}$ & 2.4 \\
\hline M37 & Nagashizu, Tokura, Minamisanriku Town & 38 & 38 & 141 & 31 & 11.3 & $\mathrm{~B}$ & a & $\mathrm{R}$ & 16 & & $\begin{array}{l}4.9 \\
5.2\end{array}$ & 4.6 & $\begin{array}{l}4.0 \\
4.2 \\
\end{array}$ & 2.4 \\
\hline M38 & $\begin{array}{l}\text { Kozashi, Kitakamicho-Jusanhama, Ishinomaki } \\
\text { City }\end{array}$ & 38 & 36 & 141 & 30 & 14.7 & A & $\mathrm{a}$ & I & 15 & & & 4.6 & & 4.8 \\
\hline M39 & $\begin{array}{c}\text { Aikawa, Kitakamicho-Jusanhama, Ishinomaki } \\
\text { City }\end{array}$ & 38 & 36 & 141 & 30 & 14.9 & A & a & $\mathrm{R}$ & 15 & & & 5.5 & & 4.8 \\
\hline M40 & $\begin{array}{c}\text { Kodomari, Kitakamicho-Jusanhama, Ishinomaki } \\
\text { City }\end{array}$ & 38 & 36 & 141 & 30 & 12.0 & A & $\mathrm{a}$ & $\mathrm{R}$ & & & & 5.0 & 5.6 & 4.5 \\
\hline M41 & $\begin{array}{c}\text { Omuro, Kitakamicho-Jusanhama, Ishinomaki } \\
\text { City } \\
\end{array}$ & 38 & 36 & 141 & 30 & 14.3 & $\mathrm{C}$ & $\mathrm{a}$ & I & 13 & & & $\begin{array}{l}3.5 \\
3.7 \\
\end{array}$ & $\begin{array}{l}5.8 \\
5.8 \\
\end{array}$ & 3.0 \\
\hline M42 & \begin{tabular}{|c|} 
Shirahama, Kitakamicho-Jusanhama, Ishinomaki \\
City
\end{tabular} & 38 & 35 & 141 & 28 & 15.7 & $\bar{A}$ & $\mathrm{a}$ & I & 9 & & & 3.2 & 4.1 & 2.1 \\
\hline M50 & Ogatsucho-Naburi, Ishinomaki City & 38 & 32 & 141 & 30 & 14.2 & B & a & $\mathrm{R}$ & 11 & & & $\begin{array}{l}2.7 \\
3.3\end{array}$ & 4.0 & 4.2 \\
\hline M51 & Ogatsucho-Funakoshi, Ishinomaki City & 38 & 32 & 141 & 31 & 12.6 & $\mathrm{~B}$ & a & $\mathrm{R}$ & & & & $\begin{array}{c}3.7 \\
4\end{array}$ & 4.0 & 4.5 \\
\hline M52 & Ogatsucho-Osu, Ishinomaki City & 38 & 31 & 141 & 32 & 11.4 & $\mathrm{~B}$ & $\mathrm{a}$ & $\mathrm{R}$ & & & & 3.8 & 4.2 & \\
\hline M53 & Ogatsucho-Kuwanohama, Ishinomaki City & 38 & 30 & 141 & 32 & 11.8 & A & $\mathrm{a}$ & I & & & & 2.3 & $\begin{array}{l}1.9 \\
1.9 \\
1.9 \\
\end{array}$ & 1.5 \\
\hline M54 & \multirow[t]{2}{*}{ Ogatsucho-Tachihama, Ishinomaki City } & 38 & 30 & 141 & 31 & 11.7 & $\mathrm{~B}$ & $\mathrm{a}$ & $\mathrm{R}$ & & & & 2.0 & $\begin{array}{l}1.3 \\
1.8\end{array}$ & 1.8 \\
\hline M55 & & 38 & 31 & 141 & 31 & 9.6 & $\mathrm{~B}$ & $\mathrm{a}$ & $\mathrm{R}$ & & & & & $\begin{array}{l}1.9 \\
2.2 \\
2.2\end{array}$ & \\
\hline M56 & Ogatsucho-Myojin, Ishinomaki City & 38 & 31 & 141 & 29 & 12.3 & B & $\mathrm{a}$ & $\mathrm{R}$ & 8 & & & 2.0 & $\begin{array}{l}2.2 \\
2.2\end{array}$ & 1.8 \\
\hline M57 & $\begin{array}{c}\text { Funatoshinmei, Ogatsucho-Ogatsu, Ishinomaki } \\
\text { City }\end{array}$ & 38 & 31 & 141 & 28 & 15.3 & $\mathrm{~A}$ & $\mathrm{a}$ & $\mathrm{R}$ & 10 & & $\begin{array}{l}2.9 \\
3.0 \\
3.1 \\
3.2 \\
3.6 \\
3.8 \\
4.8\end{array}$ & $\begin{array}{c}3.5 \\
3.65 \\
3.90 \\
3.98\end{array}$ & $\begin{array}{l}3.3 \\
3.4 \\
3.4 \\
3.7 \\
3.8 \\
4.2 \\
4.3 \\
4.5 \\
5.7\end{array}$ & \\
\hline M58 & Karakuwa, Ogatsucho-Ogatsu, Ishinomaki City & 38 & 31 & 141 & 29 & 10.1 & $\mathrm{C}$ & $\mathrm{a}$ & I & 6 & & & 2.1 & $\begin{array}{l}1.9 \\
1.9 \\
\end{array}$ & 1.8 \\
\hline M59 & $\begin{array}{c}\text { Wakehama, Ogatsucho-Wakehama, Ishinomaki } \\
\text { City }\end{array}$ & 38 & 30 & 141 & 29 & 13.7 & $\mathrm{~B}$ & a & $\mathrm{R}$ & 7 & & & 1.8 & $\begin{array}{l}1.9 \\
1.9\end{array}$ & 1.5 \\
\hline M60 & $\begin{array}{c}\text { Namiita, Ogatsucho-Wakehama, Ishinomaki } \\
\text { City }\end{array}$ & 38 & 29 & 141 & 29 & 14.5 & $\mathrm{~A}$ & a & $\mathrm{R}$ & 8 & & & 2.1 & $\begin{array}{l}2.5 \\
2.5 \\
\end{array}$ & 1.5 \\
\hline M61 & Ishihama, Onagawa Town & 38 & 27 & 141 & 28 & 16.7 & $\mathrm{~A}$ & $\mathrm{a}$ & $\mathrm{R}$ & 8 & & & 2.6 & $\begin{array}{l}2.2 \\
2.6 \\
\end{array}$ & 2.4 \\
\hline M62 & Oishiharahama, Onagawa Town & 38 & 24 & 141 & 28 & 13.0 & $\mathrm{~B}$ & $\mathrm{a}$ & $\mathrm{R}$ & & & & & $\begin{array}{l}2.2 \\
2.2 \\
\end{array}$ & 2.4 \\
\hline M65 & Samenoura, Ishinomaki City & 38 & 23 & 141 & 29 & 17.9 & $\mathrm{C}$ & $\mathrm{a}$ & I & 10 & & 2.6 & 5.0 & 3.2 & 4.8 \\
\hline M66 & Oyagawahama, Ishinomaki City & 38 & 22 & 141 & 29 & 17.8 & B & $\mathrm{a}$ & $\mathrm{R}$ & & & 2.0 & & $\begin{array}{l}2.8 \\
4.0\end{array}$ & 5.2 \\
\hline M67 & \multirow[t]{2}{*}{ Yagawahama, Ishinomaki City } & 38 & 22 & 141 & 29 & 18.7 & $\mathrm{C}$ & $\mathrm{a}$ & $\mathrm{I}$ & \multirow[t]{2}{*}{11} & \multirow{2}{*}{\multicolumn{2}{|c|}{3.0}} & \multirow[t]{2}{*}{5.2} & 4.0 & \multirow[t]{2}{*}{4.8} \\
\hline M68 & & 38 & 22 & 141 & 29 & 21.2 & $\mathrm{~B}$ & $\mathrm{a}$ & $\mathrm{R}$ & & & & & 7.0 & \\
\hline
\end{tabular}


Table 1

continued

\begin{tabular}{|c|c|c|c|c|c|c|c|c|c|c|c|c|c|c|c|}
\hline \multirow[t]{2}{*}{ No. } & \multirow{2}{*}{$\begin{array}{l}\text { Location } \\
\text { Name }\end{array}$} & \multicolumn{2}{|c|}{ Latitude } & \multicolumn{2}{|c|}{ Longitude } & \multirow{2}{*}{$\begin{array}{c}2011 \text { height }^{\mathrm{a}} \\
(\mathrm{m})\end{array}$} & \multirow[t]{2}{*}{ rel. $^{\mathrm{b}}$} & \multirow[t]{2}{*}{ acc. $^{c}$} & \multirow[t]{2}{*}{ type } & \multicolumn{3}{|c|}{1896 height $^{\mathrm{d}}$} & \multicolumn{3}{|c|}{1933 height $^{\mathrm{e}}$} \\
\hline & & deg & $\min$ & deg & $\min$. & & & & & $\begin{array}{c}\text { Iki } \\
\text { (feet) }\end{array}$ & $\begin{array}{c}\text { Yaman } \\
\mathrm{a}\end{array}$ & $\begin{array}{c}\text { Matsuo } \\
(\mathrm{m})\end{array}$ & $\begin{array}{l}\text { ERI } \\
(\mathrm{m})\end{array}$ & \begin{tabular}{|c|}
$\begin{array}{c}\text { Matsuo } \\
(\mathrm{m})\end{array}$ \\
\end{tabular} & $\begin{array}{l}\text { Kunitomi } \\
\text { (m) }\end{array}$ \\
\hline M69 & \multirow[t]{2}{*}{ Tomarihama, Ishinomaki City } & 38 & 22 & 141 & 31 & 12.4 & $\mathrm{~A}$ & $\mathrm{a}$ & $\mathrm{R}$ & \multirow[t]{2}{*}{$\mathrm{x} 20$} & & & \multirow[t]{2}{*}{3.7} & & \\
\hline M70 & & 38 & 22 & 141 & 31 & 12.2 & A & $\mathrm{a}$ & $\mathrm{R}$ & & & & & & \\
\hline M71 & Kugunarihama, Ishinomaki City & 38 & 19 & 141 & 30 & 6.3 & $\mathrm{~A}$ & $\mathrm{a}$ & I & & & & 2.1 & & 1.8 \\
\hline M72 & Koamikurahama, Ishinomaki City & 38 & 21 & 141 & 27 & 12.9 & $\mathrm{~B}$ & $\mathrm{a}$ & $\mathrm{R}$ & 7 & & & 3.0 & 2.9 & 3.0 \\
\hline M73 & Fukkiura, Ishinomaki City & 38 & 21 & 141 & 27 & 8.2 & $\mathrm{~B}$ & $\mathrm{a}$ & $\mathrm{R}$ & & & & 2.7 & & 1.2 \\
\hline M74 & Kitsunezakihama, Ishinomaki City & 38 & 21 & 141 & 25 & 8.6 & $\mathrm{~B}$ & $\mathrm{a}$ & $\mathrm{R}$ & $\mathrm{x} 2-3$ & & & & & \\
\hline M75 & Kozumihama, Ishinomaki City & 38 & 22 & 141 & 27 & 8.5 & $\mathrm{~B}$ & $\mathrm{a}$ & I & & & & 2.9 & 3.5 & 2.7 \\
\hline M76 & Momonoura, Ishinomaki City & 38 & 24 & 141 & 26 & 12.5 & $\mathrm{~B}$ & $\mathrm{a}$ & $\mathrm{R}$ & 4 & & & 1.2 & & 1.2 \\
\hline
\end{tabular}

$R$ runup height; $I$ inundation height; $P$ tsunami height in ports

a 2011 heights above sea level at time of maximum tsunami

b Rel.: reliability, A: most reliable based on clear physical evidence or eyewitness account; B: mostly based on natural traces; C: least reliable based on equivocal evidence

c Acc.: accuracy, a: measurement error $<0.2 \mathrm{~m}$; b $0.2 \leq$ error $\leq 0.5 \mathrm{~m}$; c error $>0.5 \mathrm{~m}$

${ }^{\mathrm{d}}$ Iki: runup heights taken from IKI (1897). 1 foot $=0.305 \mathrm{~m}$. $\Delta$ : visual measurements; $x$ eyewitness accounts; Yamana: measured by YAMANA (1896) reproduced by Unohana and Ota (1988). 1 shaku $=0.303 \mathrm{~m}$, Matsuo: taken from Matsuo (1933, 1934) measured with 1933 heights based on eyewitness accounts

e ERI: taken from EarthquaKe Research Institute (1934), Matsuo: taken from Matsuo (1933, 1934), Kunitomi: taken from Kunitomi (1933)

2011 inundation tsunami height was $15 \mathrm{~m}$ (I72). On the back cliff behind the icehouse, two white markers indicate the heights of the 1896 (15 m; IKI 1897) and 1933 (10 m; Earthquake Research Institute 1934) Sanriku tsunamis (Fig. 17). These show that the 2011 tsunami was larger than the 1933 tsunami, but similar to the 1896 tsunami. According to YAMASHita (2003), 1,867 of 2,248 residents in the affected area were killed by the 1896 tsunami (fatality rate $83 \%$ ), and 911 among 2,773 residents died during the 1933 tsunami (fatality rate $32 \%$ ). The 2011 tsunami killed $\sim 200$ of 4,434 (fatality rate $\sim 5 \%$ ). The 1960 tsunami heights were 2-3 m as reported by CFI (1961), and $2 \mathrm{~m}$ as reported by JMA (1961), and the coastal levee completely protected the residential area from the 1960 and 2010 Chilean tsunamis.

At Akahama in Otsuchi Town, the 2011 tsunami was larger than the other historical tsunamis. Most of the houses were swept away by the 2011 tsunami. The International Coastal Research Center, Atmosphere and Ocean Research Institute of the University of Tokyo was severely damaged up to the third floor, indicating inundation heights of $\sim 12 \mathrm{~m}$ (I121, I122). The runup height behind of the building was measured as $13 \mathrm{~m}$ (I120). During the 1896 tsunami, a tsunami height of $6 \mathrm{~m}$, reportedly caused two houses to collapse, 16 to be washed away, and 26 fatalities. The 1933 tsunami height was $5 \mathrm{~m}$, while the 1960 tsunami height was $3 \mathrm{~m}$.

At Sanrikucho-Okirai in Ofunato City, the 2011, 1896, and 1933 tsunamis caused similar inundations and runup heights; however, 1960 tsunami height was much smaller. The 2011 tsunami inundated areas up to $\sim 150 \mathrm{~m}$ from the coast near Horei Station of the Sanriku Railway. Tsunami inundation would have been larger if the railway track had not been raised to a height of $\sim 15 \mathrm{~m}$. The 2011 runup heights ranged from 15 to $18 \mathrm{~m}$ (I130-I132). The 1896 tsunami completely destroyed a coastal levee with a height of 15 shaku $(\sim 5 \mathrm{~m})$, inundating areas up to $500 \mathrm{ken}$ $(\sim 900 \mathrm{~m} ; 1 \mathrm{ken}=1.818 \mathrm{~m})$ from the coast with a reported tsunami height of $15 \mathrm{~m}$. At the time of the 1933 tsunami, the inundation distance was 300-400 m with reported tsunami heights of 4-12 m. The 1960 tsunami height was $2 \mathrm{~m}$ (CFI 1961).

At Sanrikucho-Ryori in Ofunato City, the 2011 tsunami height was lower than the 1896 and 1933 Sanriku tsunami heights, but higher than the 1960 tsunami. The 1896 tsunami height was reported as 130-180 shaku (approximately 39-55 m; YAMANA 1896), 72 feet (22 m; IKI 1897), or 38 m (Matsuo 1933) (Fig. 18), and it killed 204 of 240 residents. According to Matsuo (1933), the maximum heights of the 1896 Sanriku tsunami (38 m) and 1933 Sanriku tsunami $(29 \mathrm{~m})$ were recorded here. The 2011 runup 
Table 2

Tsunami heights for Sanriku coasts of Aomori, Iwate, and Miyagi prefectures and Pacific coasts of Ibaraki and Chiba prefectures from the 1960, 2010, and 2011 earthquakes

\begin{tabular}{|c|c|c|c|c|c|c|c|c|c|c|c|c|c|c|c|c|c|}
\hline \multirow[t]{2}{*}{ No. } & \multirow[t]{2}{*}{ Location Name } & \multicolumn{2}{|c|}{ Latitude } & \multicolumn{2}{|c|}{ Longitude } & \multirow{2}{*}{$\begin{aligned} & \begin{array}{r}2011 \\
\text { height }^{\mathrm{a}}\end{array}(\mathrm{m}) \\
&\end{aligned}$} & \multirow[t]{2}{*}{ rel. $^{b}$} & \multirow[t]{2}{*}{ acc. $^{c}$} & \multirow[t]{2}{*}{ type } & \multicolumn{6}{|c|}{1960 height } & \multicolumn{2}{|c|}{2010 height } \\
\hline & & deg & $\min$ & deg & $\min$ & & & & & $\begin{aligned} \mathrm{CFI}^{\mathrm{d}} \\
(\mathrm{m})\end{aligned}$ & \begin{tabular}{|c|} 
CFI \\
Corrected \\
$(\mathrm{m})$
\end{tabular} & $\begin{array}{l}\text { CFI } \\
\text { acc. }^{f}\end{array}$ & $\begin{array}{c}\mathrm{JMA}^{\mathrm{g}} \\
(\mathrm{m})\end{array}$ & $\begin{array}{r}\text { JMA } \\
\text { datum }\end{array}$ & \begin{tabular}{|c|} 
JMA \\
Corrected \\
$(\mathrm{m})$
\end{tabular} \mid & $\underset{(\mathrm{m})}{\text { Tsuji/Imai }^{\mathrm{h}}}$ & type \\
\hline $\mathrm{Al}$ & $\begin{array}{l}\text { Shimomekurakubo, Samemachi, } \\
\text { Hachinohe City (Hachinohe Fishing } \\
\text { Port, Ebisuhama District) }\end{array}$ & 40 & 32 & 141 & 34 & 6.1 & $\mathrm{~A}$ & $\mathrm{a}$ & I & 4.2 & 4.2 & 4 & & & & 0.9 & $T$ \\
\hline $\mathrm{A} 2$ & $\begin{array}{l}\text { Yoboishi, Samemachi, Hachinohe City } \\
\text { (Tanesashi Fishing Port) }\end{array}$ & 40 & 30 & 141 & 37 & 9.3 & $\mathrm{~A}$ & a & I & 3.9 & 3.9 & 3 & 4.1 & tp & 4.1 & & \\
\hline $\mathrm{A} 3$ & $\begin{array}{l}\text { Daisakutai, Samemachi, Hachinohe } \\
\text { City (Okuki Fishing Port) }\end{array}$ & 40 & 30 & 141 & 38 & 9.9 & $\mathrm{~A}$ & a & I & $\begin{array}{l}3.7 \\
5.6\end{array}$ & $\begin{array}{l}3.7 \\
5.6\end{array}$ & $\begin{array}{l}4 \\
4\end{array}$ & 1.6 & tp & 1.6 & & \\
\hline A4 & \begin{tabular}{|c|} 
Oja, Dobutsu, Hashikami Town (Oja \\
Fishing Port) \\
\end{tabular} & 40 & 28 & 141 & 39 & 9.3 & $\mathrm{~A}$ & $\mathrm{a}$ & $\mathrm{R}$ & 4.4 & 4.4 & 4 & 2.6 & tp & 2.6 & & \\
\hline A5 & $\begin{array}{l}\text { Kominato, Dobutsu, Hashikami Town } \\
\text { (Kominato Fishing Port) }\end{array}$ & 40 & 27 & 141 & 40 & 6.0 & $\mathrm{~A}$ & $\mathrm{a}$ & $\mathrm{R}$ & $4.1-5.2$ & $\begin{array}{l}4.1 \\
-5.2\end{array}$ & 2 & 3.6 & tp & 3.6 & & \\
\hline I1 & $\begin{array}{l}\text { Taneichi (Kadonohama), Hirono Town } \\
\text { (Kadonohama Fishing Port) }\end{array}$ & 40 & 27 & 141 & 41 & 7.0 & B & $\mathrm{a}$ & $\mathrm{R}$ & 4.9 & 4.9 & 2 & 2.3 & tp & 2.3 & & \\
\hline I2 & $\begin{array}{l}\text { Taneichi, Hirono Town (Taneichi } \\
\text { Fishing Port) }\end{array}$ & 40 & 24 & 141 & 43 & 6.8 & $\mathrm{~B}$ & $\mathrm{a}$ & I & 6.2 & 6.2 & 2 & 2.2 & tp & 2.2 & & \\
\hline 13 & Taneichi, Hirono Town (Yagi Port) & 40 & 21 & 141 & 46 & 7.8 & B & $\mathrm{b}$ & I & $\begin{array}{l}3.0 \\
3.5\end{array}$ & $\begin{array}{l}3.0 \\
3.5\end{array}$ & $\begin{array}{l}4 \\
4 \\
\end{array}$ & 2.4 & tp & 2.4 & & \\
\hline 15 & $\begin{array}{c}\text { Nakano, Hirono Town (Koge Fishing } \\
\text { Port, Koge District) }\end{array}$ & 40 & 18 & 141 & 48 & 14.8 & $\mathrm{~B}$ & $\mathrm{a}$ & $\mathrm{R}$ & & & & 3.0 & tp & 3.0 & & \\
\hline 17 & $\begin{array}{l}\text { Samuraihamacho-Mukaicho, Kuji City } \\
\text { (Kawatsunai Fishing Port, Kawatsunai } \\
\text { District) }\end{array}$ & 40 & 16 & 141 & 49 & 12.9 & $\mathrm{~B}$ & a & $\mathrm{R}$ & & & & 1.3 & tp & 1.3 & & \\
\hline I11 & $\begin{array}{l}\text { Osanaicho (Tamanowaki), Kuji City } \\
\text { (Tamanowaki Fishing Port) }\end{array}$ & 40 & 11 & 141 & 48 & 10.7 & $\mathrm{~A}$ & $\mathrm{a}$ & $\mathrm{R}$ & 4.0 & 4.0 & 4 & 3.6 & tp & 3.6 & $\begin{array}{l}1.2 \\
1.2\end{array}$ & $\begin{array}{l}\mathrm{T} \\
\mathrm{I}\end{array}$ \\
\hline $\mathrm{II2}$ & $\begin{array}{l}\text { Ubecho (Kosode), Kuji City (Kosode } \\
\text { Fishing Port, Kosode District) }\end{array}$ & 40 & 10 & 141 & 51 & 12.0 & $\mathrm{~B}$ & $\mathrm{a}$ & I & & & & 3.8 & tp & 3.8 & & \\
\hline I13 & Ubecho (Kosode), Kuji City & 40 & 10 & 141 & 51 & 8.2 & $\mathrm{~A}$ & $\mathrm{a}$ & $\mathrm{R}$ & & & & & & & & \\
\hline 115 & $\begin{array}{c}\text { Ubecho (Kuki), Kuji City (Kuki } \\
\text { Fishing Port) } \\
\end{array}$ & 40 & 8 & 141 & 51 & 14.6 & A & $\mathrm{b}$ & I & $\begin{array}{l}4.8 \\
5.0 \\
\end{array}$ & $\begin{array}{l}4.8 \\
5.0 \\
\end{array}$ & $\begin{array}{l}3 \\
3 \\
\end{array}$ & 4.1 & tp & 4.1 & & \\
\hline I16 & Noda, Noda Village (Noda Fishing & 40 & 7 & 141 & 50 & 22.8 & $\mathrm{C}$ & $\mathrm{a}$ & $\mathrm{R}$ & 4.9 & 4.9 & 1 & 5.2 & tp & 5.2 & & \\
\hline I17 & & 40 & 7 & 141 & 50 & 16.4 & $\mathrm{~B}$ & $\mathrm{a}$ & $\mathrm{R}$ & & & & & & & & \\
\hline I18 & & 40 & 7 & 141 & 50 & 18.5 & $\mathrm{~B}$ & $\mathrm{a}$ & $\mathrm{R}$ & & & & & & & & \\
\hline 119 & Noda (Maita), Noda Village & 40 & 6 & 141 & 50 & 32.0 & B & $\mathrm{b}$ & $\mathrm{R}$ & 4.9 & 4.7 & 4 & & & & & \\
\hline 120 & $\begin{array}{l}\text { Tamagawa, Noda Village (Noda } \\
\text { Tamagawa Station) }\end{array}$ & 40 & 5 & 141 & 50 & 26.6 & B & $\mathrm{a}$ & $\mathrm{R}$ & $\begin{array}{l}5.9 \\
5.9\end{array}$ & $\begin{array}{l}5.7 \\
5.7\end{array}$ & $\begin{array}{l}4 \\
4\end{array}$ & 8.1 & tp & 7.9 & & \\
\hline 121 & Tamagawa (Shimoakka), Noda Village & 40 & 3 & 141 & 51 & 11.8 & $\mathrm{~B}$ & $\mathrm{a}$ & $\mathrm{R}$ & $\begin{array}{l}2.7 \\
2.7 \\
2.8 \\
\end{array}$ & $\begin{array}{l}2.5 \\
2.5 \\
2.6\end{array}$ & $\begin{array}{l}4 \\
4 \\
4\end{array}$ & & & & & \\
\hline 122 & Otanabe, Fudai Village (Otanabe & 40 & 1 & 141 & 54 & 8.4 & $\mathrm{~A}$ & $\mathrm{c}$ & I & 2.5 & 2.3 & 4 & 2.4 & tp & 2.2 & & \\
\hline I23 & Fishing Port) & 40 & 1 & 141 & 54 & 8.9 & $\mathrm{~A}$ & $\mathrm{a}$ & $\mathrm{I}$ & 2.6 & 2.4 & 3 & & & & & \\
\hline $\mathrm{I} 24$ & & 40 & 1 & 141 & 54 & 12.4 & $\mathrm{C}$ & $\mathrm{a}$ & $\mathrm{R}$ & & & & & & & & \\
\hline 125 & & 40 & 1 & 141 & 54 & 10.6 & $\mathrm{~B}$ & $\mathrm{a}$ & $\mathrm{R}$ & & & & & & & & \\
\hline 134 & Raga, Tanohata Village & 39 & 56 & 141 & 56 & 23.9 & $\mathrm{~A}$ & $\mathrm{a}$ & $\mathrm{I}$ & & & & $\mathrm{x} 1.0$ & tp & 0.8 & & \\
\hline 135 & & 39 & 56 & 141 & 56 & 22.9 & A & $\mathrm{a}$ & $\mathrm{R}$ & & & & & & & & \\
\hline 136 & & 39 & 56 & 141 & 56 & 27.8 & $\mathrm{~B}$ & $\mathrm{a}$ & $\mathrm{R}$ & & & & & & & & \\
\hline 137 & & 39 & 56 & 141 & 56 & 24.5 & $\mathrm{~B}$ & $\mathrm{a}$ & $\mathrm{R}$ & & & & & & & & \\
\hline 138 & Raga (Hiraiga), Tanohata Village & 39 & 56 & 141 & 56 & 17.5 & $\mathrm{~B}$ & $\mathrm{a}$ & $\mathrm{R}$ & 2.3 & 2.1 & 3 & $\mathrm{x} 1.0$ & tp & 0.8 & & \\
\hline 139 & & 39 & 56 & 141 & 56 & 16.5 & $\mathrm{~B}$ & $\mathrm{a}$ & $\mathrm{R}$ & 2.3 & 2.1 & 4 & & & & & \\
\hline 140 & Wano, Tanohata Village & 39 & 56 & 141 & 56 & 19.0 & B & $\mathrm{a}$ & I & $\begin{array}{l}2.8 \\
3.0\end{array}$ & $\begin{array}{l}2.6 \\
2.8\end{array}$ & $\begin{array}{l}4 \\
3\end{array}$ & & & & & \\
\hline I41 & Shimanokoshi, Tanohata Village & 39 & 55 & 141 & 56 & 19.9 & B & $\mathrm{a}$ & $\mathrm{R}$ & 2.0 & 1.8 & 4 & $\times 1.0$ & tp & 0.8 & & \\
\hline 142 & & 39 & 55 & 141 & 56 & 22.0 & A & $\mathrm{a}$ & $\mathrm{R}$ & 2.1 & 1.9 & 4 & & & & & \\
\hline 143 & Omoto, Iwaizumi Town & 39 & 51 & 141 & 58 & 10.3 & $\mathrm{~B}$ & $\mathrm{a}$ & $\mathrm{R}$ & $\begin{array}{l}2.8 \\
4.0\end{array}$ & $\begin{array}{l}2.6 \\
3.8\end{array}$ & $\begin{array}{l}4 \\
4\end{array}$ & $\mathrm{x} 1.0$ & tp & 0.8 & & \\
\hline 147 & & 39 & 51 & 141 & 58 & 20.4 & B & $\mathrm{a}$ & $\mathrm{R}$ & 4.4 & 4.2 & 4 & & & & & \\
\hline 151 & Moshi, Omoto, Iwaizumi Town & 39 & 50 & 141 & 58 & 22.3 & $\mathrm{~B}$ & $\mathrm{a}$ & $\mathrm{R}$ & 2.8 & 2.6 & 4 & & & & & \\
\hline 152 & & 39 & 50 & 141 & 58 & 22.2 & $\mathrm{~B}$ & $\mathrm{a}$ & $\mathrm{R}$ & & & & & & & & \\
\hline 153 & Taro-Shimosettai, Miyako City & 39 & 49 & 141 & 59 & 21.8 & $\mathrm{~B}$ & $\mathrm{a}$ & $\mathrm{R}$ & 2.6 & 2.4 & 4 & $\mathrm{x} 1.0$ & tp & 0.8 & & \\
\hline 154 & & 39 & 49 & 141 & 59 & 27.9 & $\mathrm{~B}$ & $\mathrm{a}$ & $\mathrm{R}$ & & & & & & & & \\
\hline 155 & & 39 & 49 & 141 & 59 & 22.3 & $\mathrm{~A}$ & $\mathrm{a}$ & I & & & & & & & & \\
\hline $\mathrm{I} 60$ & $\begin{array}{c}\text { Taro-Aonotakiminami, Miyako City } \\
\text { (Aonotaki Fishing Port) }\end{array}$ & 39 & 46 & 141 & 59 & 34.7 & $\mathrm{~B}$ & $\mathrm{~b}$ & $\mathrm{R}$ & 2.4 & 2.2 & 4 & & & & & \\
\hline I61 & Taro-Otobeno, Miyako City & 39 & 45 & 141 & 59 & 22.9 & $\mathrm{~B}$ & $\mathrm{a}$ & $\mathrm{R}$ & 2.3 & 2.1 & 4 & & & & & \\
\hline 162 & & 39 & 45 & 141 & 59 & 16.5 & $\mathrm{~B}$ & $\mathrm{a}$ & $\mathrm{R}$ & & & & & & & & \\
\hline I63 & & 39 & 45 & 141 & 59 & 23.3 & $\mathrm{~B}$ & $\mathrm{a}$ & $\mathrm{R}$ & & & & & & & & \\
\hline 164 & & 39 & 45 & 141 & 59 & 30.1 & B & $\mathrm{a}$ & $\mathrm{R}$ & & & & & & & & \\
\hline 165 & & 39 & 45 & 141 & 59 & 34.1 & $\mathrm{~B}$ & $\mathrm{a}$ & $\mathrm{R}$ & & & & & & & & \\
\hline 166 & Taro-Wano, Miyako City & 39 & 45 & 142 & 0 & 30.7 & $\mathrm{~B}$ & $\mathrm{a}$ & $\mathrm{R}$ & 2.5 & 2.3 & 4 & & & & & \\
\hline 167 & & 39 & 45 & 141 & 59 & 27.4 & $\mathrm{~B}$ & $\mathrm{a}$ & $\mathrm{R}$ & & & & & & & & \\
\hline 170 & Taro-Nohara, Miyako City & 39 & 44 & 141 & 59 & 14.8 & A & $\mathrm{c}$ & I & 1.8 & 1.6 & 4 & & & & & \\
\hline
\end{tabular}


Table 2

continued

\begin{tabular}{|c|c|c|c|c|c|c|c|c|c|c|c|c|c|c|c|c|c|}
\hline \multirow[t]{2}{*}{ No. } & \multirow[t]{2}{*}{ Location Name } & \multicolumn{2}{|c|}{ Latitude } & \multicolumn{2}{|c|}{$\begin{array}{l}\text { Longitude } \\
\end{array}$} & \multirow{2}{*}{\begin{tabular}{|c|}
$\begin{array}{r}2011 \\
\text { height }\end{array}$ \\
$(\mathrm{m})$
\end{tabular}} & \multirow[t]{2}{*}{ rel. $^{\mathrm{b}}$} & acc. $^{c}$ & type & & & & reight & & & $2010 \mathrm{~h}$ & \\
\hline & & $\operatorname{deg}$ & $\min$ & $\operatorname{deg}$ & $\min$ & & & & & $\begin{array}{c}\mathrm{CFI}^{\mathrm{d}} \\
(\mathrm{m})\end{array}$ & \begin{tabular}{|c|} 
CFI \\
$\begin{array}{c}\text { Correctede } \\
\text { (m) }\end{array}$
\end{tabular} & $\begin{array}{l}\text { CFI } \\
\text { acc. }{ }^{f}\end{array}$ & $\begin{array}{c}\mathrm{JMA}^{8} \\
(\mathrm{~m})\end{array}$ & $\begin{aligned} \text { JMA } \\
\text { datum }\end{aligned}$ & \begin{tabular}{|c|} 
JMA \\
Correctede \\
$(\mathrm{m})$
\end{tabular} & 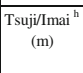 & type \\
\hline 171 & Taro-Aozari, Miyako City & 39 & 44 & 141 & 59 & 19.0 & B & $\mathrm{b}$ & $\mathrm{R}$ & 1.8 & 1.6 & 4 & & & & & \\
\hline 172 & & 39 & 44 & 141 & 59 & 14.8 & A & $\mathrm{c}$ & I & $\begin{array}{l}2.6 \\
2.7\end{array}$ & $\begin{array}{l}2.4 \\
2.5\end{array}$ & $\begin{array}{l}4 \\
4\end{array}$ & 2.6 & tp & 2.4 & & \\
\hline 174 & Taro-Nishimukaiyama, Miyako City & 39 & 43 & 141 & 58 & 25.5 & $\mathrm{~B}$ & $\mathrm{a}$ & $\mathrm{R}$ & 2.8 & 2.6 & 3 & & & & & \\
\hline 175 & (Kashinai Fishing Port) & 39 & 42 & 141 & 59 & 17.5 & B & $\mathrm{a}$ & $\mathrm{R}$ & & & & & & & & \\
\hline 176 & $\begin{array}{l}\text { Taro-Kashinai, Miyako City (Kashinai } \\
\text { Fishing Port) }\end{array}$ & 39 & 42 & 141 & 58 & 21.8 & B & $\mathrm{a}$ & $\mathrm{R}$ & & & & & & & & \\
\hline $\mathrm{I} 80$ & Sakiyama (Onappe), Miyako City & 39 & 42 & 141 & 58 & 15.1 & A & $\mathrm{a}$ & $\mathrm{R}$ & 2.1 & 1.9 & 4 & & & & & \\
\hline I81 & & 39 & 42 & 141 & 58 & 14.8 & $\mathrm{~B}$ & $\mathrm{a}$ & $\mathrm{R}$ & 3.1 & 2.9 & 4 & & & & & \\
\hline 182 & & 39 & 41 & 141 & 58 & 12.6 & $\mathrm{~B}$ & $\mathrm{a}$ & $\mathrm{I}$ & & & & & & & & \\
\hline 183 & & 39 & 41 & 141 & 58 & 17.6 & $\mathrm{~B}$ & $\mathrm{a}$ & $\mathrm{R}$ & & & & & & & & \\
\hline 184 & & 39 & 41 & 141 & $\begin{array}{l}58 \\
58 \\
\end{array}$ & 20.6 & $\mathrm{~B}$ & $\mathrm{a}$ & $R$ & & & & & & & & \\
\hline $\begin{array}{l}185 \\
186\end{array}$ & Kuwagasaki (Nakamachi), Miyako City & $\begin{array}{l}39 \\
39\end{array}$ & $\begin{array}{l}41 \\
39\end{array}$ & $\begin{array}{l}141 \\
141\end{array}$ & $\begin{array}{l}58 \\
58\end{array}$ & $\begin{array}{l}26.0 \\
8.2\end{array}$ & $\begin{array}{ll}\mathrm{B} \\
\mathrm{A}\end{array}$ & $\frac{a}{a}$ & $\begin{array}{ll}R \\
I\end{array}$ & 1.8 & 1.6 & 4 & 2.0 & tp & 1.8 & 0.7 & $\begin{array}{c}T \\
\end{array}$ \\
\hline & & & & & & & & & & & & & 2.2 & & 2.0 & & \\
\hline I87 & Rinkodori, Miyako City & 39 & 38 & 141 & 58 & 11.1 & $\mathrm{C}$ & $\mathrm{a}$ & I & 2.1 & 1.9 & 4 & 2.2 & tp & 2.0 & & \\
\hline 188 & $\begin{array}{l}\text { Tsugaruishi (Norinowaki), Miyako } \\
\text { City }\end{array}$ & 39 & 35 & 141 & 57 & 11.6 & $\mathrm{~B}$ & $\mathrm{a}$ & $\mathrm{R}$ & 6.3 & 6.1 & 4 & 4.3 & tp & 4.1 & $\begin{array}{l}2.0 \\
2.1\end{array}$ & $\begin{array}{l}I^{2} \\
\end{array}$ \\
\hline I89 & Akamae, Miyako City & 39 & 36 & 141 & 58 & 9.5 & $\mathrm{~B}$ & $\mathrm{a}$ & $\mathrm{R}$ & 4.6 & 4.4 & 4 & 5.2 & tp & 5.0 & & \\
\hline $\mathrm{I} 90$ & & 39 & 36 & 141 & 58 & 14.0 & $\mathrm{~B}$ & $\mathrm{a}$ & $\mathrm{R}$ & 5.2 & 5.0 & 2 & & & & & \\
\hline I91 & Omoe (Tatehama), Miyako City & 39 & 38 & 142 & 1 & 22.5 & $\mathrm{~B}$ & a & $\mathrm{R}$ & & & & 1.7 & tp & 1.5 & & \\
\hline 192 & & 39 & 38 & 142 & 1 & 25.2 & $\mathrm{~B}$ & a & $\mathrm{R}$ & & & & & & & & \\
\hline 193 & & 39 & 38 & 142 & 1 & 26.1 & $\mathrm{~B}$ & $\mathrm{a}$ & $\mathrm{R}$ & & & & & & & & \\
\hline 194 & Omoe (Shukuhama), Miyako City & 39 & 38 & $\frac{142}{142}$ & 1 & 25.1 & $\mathrm{~B}$ & $\frac{a}{a}$ & $\begin{array}{l}R \\
R\end{array}$ & 2.1 & 1.9 & 3 & & & & & \\
\hline$\frac{195}{196}$ & Omoe (Uiso), Miyako City & $\begin{array}{l}39 \\
39 \\
\end{array}$ & $\frac{38}{37}$ & $\frac{142}{142}$ & $\frac{1}{1}$ & $\frac{24.6}{27.0}$ & $\frac{\mathrm{B}}{\mathrm{B}}$ & $\frac{a}{a}$ & $\frac{R}{R}$ & 2.5 & 2.3 & 3 & 2.2 & tp & 2.0 & & \\
\hline 197 & & 39 & 37 & 142 & 1 & 25.4 & $\mathrm{~A}$ & $\frac{a}{a}$ & I & & & & & & & & \\
\hline 198 & & 39 & 37 & 142 & 1 & 23.2 & $\mathrm{~B}$ & $\mathrm{a}$ & $\mathrm{R}$ & & & & & & & & \\
\hline 199 & & 39 & 37 & 142 & 1 & 24.5 & $\mathrm{~A}$ & $\mathrm{a}$ & I & & & & & & & & \\
\hline 1100 & Omoe (Aramaki), Miyako City & 39 & 36 & 142 & 1 & 21.1 & $\mathrm{~B}$ & $\mathrm{a}$ & $R$ & 2.6 & 2.4 & 4 & 0.6 & tp & 0.4 & & \\
\hline II01 & & 39 & 36 & 142 & 1 & 27.7 & $\mathrm{~B}$ & $\mathrm{a}$ & $\mathrm{R}$ & & & & & & & & \\
\hline $\mathrm{I} 102$ & & 39 & 36 & 142 & 1 & 23.5 & $\mathrm{~B}$ & $\mathrm{a}$ & $\mathrm{R}$ & & & & & & & & \\
\hline 1103 & Omoe (Sato), Miyako City (Omoe & 39 & 34 & 142 & 2 & 20.1 & $\mathrm{~B}$ & $\mathrm{a}$ & $\mathrm{R}$ & 2.5 & 2.3 & 3 & 1.6 & tp & 1.4 & & \\
\hline I104 & Fishing Port) & 39 & 34 & 142 & 2 & 34.1 & $\mathrm{C}$ & $\mathrm{a}$ & $\mathrm{R}$ & 2.8 & 2.6 & 4 & & & & & \\
\hline I105 & Omoe (Aneyoshi), Miyako City & 39 & 32 & 142 & 3 & 26.5 & $\mathrm{~B}$ & $\mathrm{a}$ & $\mathrm{R}$ & 3.0 & 2.8 & 4 & 1.5 & tp & 1.3 & & \\
\hline II06 & & 39 & 32 & 142 & 3 & 22.8 & $\mathrm{~B}$ & $\mathrm{a}$ & $\mathrm{R}$ & 3.1 & 2.9 & 4 & & & & & \\
\hline I107 & & 39 & 32 & 142 & 3 & 24.5 & $\mathrm{~B}$ & $\mathrm{c}$ & $\mathrm{R}$ & & & & & & & & \\
\hline 1108 & Omoe (Chikei), Miyako City & 39 & 32 & 142 & $\frac{2}{2}$ & 29.5 & $\mathrm{~A}$ & $\mathrm{a}$ & $\mathrm{R}$ & 3.1 & 2.9 & 4 & 3.0 & tp & 2.8 & & \\
\hline I109 & & 39 & 32 & 142 & 2 & 31.2 & A & $\mathrm{a}$ & $\mathrm{R}$ & & & & & & & & \\
\hline $\mathrm{I110}$ & & 39 & 32 & 142 & 2 & 29.4 & A & $\mathrm{a}$ & $\mathrm{R}$ & & & & & & & & \\
\hline 1111 & Omoe (Ishihama), Miyako City & 39 & 31 & 142 & 2 & 20.0 & $\mathrm{~A}$ & $\mathrm{a}$ & I & $\begin{array}{l}2.6 \\
3.8\end{array}$ & 2.4 & 4 & 2.7 & tp & 2.5 & & \\
\hline I112 & Funakoshi (Ohura), Yamada Town & 39 & 27 & 142 & 0 & 8.0 & $\mathrm{~B}$ & $\mathrm{a}$ & $\mathrm{R}$ & 3.0 & 2.8 & 4 & & & & & \\
\hline 1113 & & 39 & 27 & 142 & 0 & 7.7 & A & $\mathrm{a}$ & I & & & & & & & & \\
\hline I114 & & 39 & 27 & 142 & 0 & 8.0 & $\mathrm{~B}$ & a & $\mathrm{R}$ & & & & & & & & \\
\hline 1115 & & 39 & 27 & 142 & 0 & 7.8 & $\mathrm{~B}$ & $\mathrm{a}$ & $\mathrm{R}$ & & & & & & & & \\
\hline 1118 & Funakoshi (Koyadori), Yamada Town & 39 & 26 & 142 & 1 & 26.1 & A & $\mathrm{a}$ & $R$ & 2.3 & 2.1 & 4 & & & & & \\
\hline 1119 & & 39 & 26 & 142 & 1 & 25.0 & $\mathrm{~B}$ & a & $\mathrm{R}$ & & & & & & & & \\
\hline 1120 & Akahama, Otsuchi Town & 39 & 21 & 141 & 56 & 13.3 & $\mathrm{~B}$ & $\mathrm{a}$ & $R$ & 3.4 & 3.2 & 4 & 3.6 & tp & 3.4 & & \\
\hline $\mathrm{I121}$ & Akahama, Otsuchi Town & 39 & 21 & 141 & 56 & 11.5 & $\mathrm{~A}$ & $\mathrm{a}$ & I & & & & & & & & \\
\hline $\mathrm{I1122}$ & $\begin{array}{c}\text { (International Coastal Research } \\
\text { Center, Atmosphere and Ocean } \\
\text { Research Institute, the University of } \\
\text { Tokyo) }\end{array}$ & 39 & 21 & 141 & 56 & 12.1 & $\mathrm{~A}$ & $\mathrm{a}$ & I & & & & & & & & \\
\hline 1123 & Omachi, Kamaishi City & 39 & 16 & 141 & 53 & 5.4 & A & b & I & $\begin{array}{l}2.8 \\
2.9\end{array}$ & $\begin{array}{l}2.6 \\
2.7\end{array}$ & $\begin{array}{l}4 \\
4\end{array}$ & 3.5 & tp & 3.3 & 0.5 & $\mathrm{~T}$ \\
\hline$\overline{I 124}$ & Osone, Tonicho, & 39 & 13 & 141 & 53 & 203 & $\mathrm{~A}$ & 3 & $\mathrm{R}$ & 2.9 & 2.7 & $\frac{4}{4}$ & 22 & to & 20 & & \\
\hline 1125 & Kamaishi City & 39 & 13 & $\frac{141}{141}$ & 53 & 16.7 & $\mathrm{C}$ & 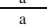 & $\frac{\pi}{I}$ & 2.2 & 2.0 & 4 & & & & & \\
\hline $\mathrm{I126}$ & & 39 & 13 & 141 & 53 & 16.8 & $\mathrm{~B}$ & $\mathrm{a}$ & $\mathrm{R}$ & & & & & & & & \\
\hline II27 & Sakuratc & 39 & 12 & 141 & 53 & 13.7 & $\mathrm{~B}$ & $\mathrm{a}$ & $x_{0}$ & & & & & & & & \\
\hline $\mathrm{II28}$ & Kamaishi City & 39 & 12 & 141 & 53 & 12.7 & $\mathrm{~B}$ & $\mathrm{a}$ & $\mathrm{R}$ & & & & & & & & \\
\hline $\mathrm{I1129}$ & Arakawa, Tonicho, Kamaishi City & 39 & 12 & 141 & 52 & 16.5 & $\mathrm{~B}$ & $\mathrm{a}$ & $\mathrm{R}$ & & & & & & & 1.2 & I \\
\hline I130 & Horei, Sanrikucho-Okirai, Ofunato & 39 & 6 & 141 & 48 & 17.8 & $\mathrm{~B}$ & $\mathrm{a}$ & $\mathrm{R}$ & 2.6 & 2.4 & 4 & & & & & \\
\hline 1131 & City & 39 & 6 & 141 & 48 & 14.8 & $\mathrm{~B}$ & $\mathrm{a}$ & $\mathrm{R}$ & & & & & & & & \\
\hline 1132 & & 39 & 6 & $\frac{141}{141}$ & 48 & 15.2 & $\mathrm{~B}$ & $\frac{a}{a}$ & $\frac{R}{R}$ & & & & & & & & \\
\hline 1133 & $\begin{array}{l}\text { Shirahama, Sanrikucho-Ryori, Ofunato } \\
\text { City }\end{array}$ & 39 & 4 & 141 & 49 & 16.8 & $\mathrm{~B}$ & $\mathrm{a}$ & $\mathrm{R}$ & $\begin{array}{l}2.9 \\
4.0\end{array}$ & $\begin{array}{l}2.7 \\
3.8\end{array}$ & $\begin{array}{l}3 \\
4\end{array}$ & $\begin{array}{l}4.3 \\
4.7\end{array}$ & tp & $\begin{array}{l}4.1 \\
4.5\end{array}$ & & \\
\hline I134 & $\begin{array}{l}\text { Okubo, Sanrikucho-Ryori, Ofunato } \\
\text { City }\end{array}$ & 39 & 3 & 141 & 49 & 21.2 & $\mathrm{~B}$ & a & $\mathrm{R}$ & & & & & & & & \\
\hline I135 & Miyanomae, Ofunatocho, Ofunato City & 39 & 2 & 141 & 43 & 7.6 & $\mathrm{~A}$ & $\mathrm{a}$ & $\mathrm{I}$ & $\begin{array}{l}2.6 \\
4.2\end{array}$ & $\begin{array}{l}2.4 \\
4.0\end{array}$ & $\begin{array}{l}4 \\
4\end{array}$ & 5.13 & $\mathrm{tp}$ & 5.0 & 0.4 & $\mathrm{~T}$ \\
\hline 1136 & $\begin{array}{l}\text { Yonesakicho, } \\
\text { Yiskre }\end{array}$ & 39 & 1 & 141 & 40 & 18.1 & $\mathrm{~B}$ & $\mathrm{~b}$ & $\mathrm{R}$ & 5.0 & 4.8 & 4 & 6.36 & tp & 6.2 & & \\
\hline M1 & \begin{tabular}{|l|} 
Karakuwacho-Baba, Kesennuma City \\
\end{tabular} & 38 & 54 & 141 & 39 & 13.1 & A & a & $\mathrm{R}$ & 2.0 & 2.2 & 2 & & & & & \\
\hline M3 & $\begin{array}{c}\text { Karakuwacho-Kakehama, Kesennuma } \\
\text { City }\end{array}$ & 38 & 53 & 141 & 40 & 15.6 & A & $\mathrm{a}$ & $\mathrm{I}$ & 3.8 & 4.0 & 2 & & & & & \\
\hline M4 & $\begin{array}{l}\text { Karakuwacho-Tsumoto, Kesennuma } \\
\text { City }\end{array}$ & 38 & 52 & 141 & 40 & 10.1 & A & $\mathrm{a}$ & I & 1.0 & 1.2 & 3 & 2.0 & tp & 2.2 & & \\
\hline M5 & \begin{tabular}{|l} 
Minamimachikaigan, Kesennuma City \\
\end{tabular} & 38 & 54 & 141 & 34 & 4.0 & A & $\mathrm{a}$ & I & 2.7 & 2.9 & 4 & 2.9 & tp & 3.1 & 0.7 & $\mathrm{R}$ \\
\hline M6 & (Kesennuma Port) & 38 & 54 & 141 & 34 & 3.7 & A & $\mathrm{a}$ & I & & & & & & & 0.9 & $\mathrm{R}$ \\
\hline M7 & & 38 & 54 & 141 & 34 & 4.1 & $\mathrm{~A}$ & $\mathrm{a}$ & I & & & & & & & 1.0 & I \\
\hline M8 & & 38 & 54 & 141 & 35 & 4.1 & $\mathrm{~A}$ & $\mathrm{a}$ & I & & & & & & & 1.2 & I \\
\hline M9 & Hajikamisuginoshita, Kesennuma City & 38 & 50 & 141 & 35 & 13.7 & $\mathrm{~A}$ & $\mathrm{~b}$ & I & 3.6 & 3.8 & 4 & & & & 0.3 & I \\
\hline
\end{tabular}


Table 2

continued

\begin{tabular}{|c|c|c|c|c|c|c|c|c|c|c|c|c|c|c|c|c|c|}
\hline \multirow[t]{2}{*}{ No. } & \multirow[t]{2}{*}{ Location Name } & \multicolumn{2}{|c|}{ Latitude } & \multicolumn{2}{|c|}{ Longitude } & \multirow{2}{*}{$\begin{array}{c}\begin{array}{r}2011 \\
\text { height }\end{array} \\
(\mathrm{m})\end{array}$} & \multirow[t]{2}{*}{ rel. $^{\mathrm{b}}$} & \multirow[t]{2}{*}{ acc. $^{c}$} & \multirow[t]{2}{*}{ type } & \multicolumn{6}{|c|}{1960 height } & \multicolumn{2}{|c|}{2010 height } \\
\hline & & deg & $\min$ & deg & $\min$ & & & & & $\begin{array}{r}\mathrm{CFI}^{\mathrm{d}} \\
(\mathrm{m})\end{array}$ & \begin{tabular}{|c|}
$\begin{array}{c}\text { CFI } \\
\text { Correctede } \\
(\mathrm{m})\end{array}$ \\
\end{tabular} & $\begin{array}{l}\text { CFI } \\
\text { acc. }^{f}\end{array}$ & $\begin{array}{c}\mathrm{JMA}^{\mathrm{g}} \\
(\mathrm{m})\end{array}$ & $\begin{array}{r}\text { JMA } \\
\text { datum }\end{array}$ & \begin{tabular}{|c|} 
JMA \\
$\begin{array}{c}\text { Correctede } \\
(\mathrm{m})\end{array}$
\end{tabular} & $=\underset{(\mathrm{m})}{\text { Tsuji//mai }}{ }^{\mathrm{h}}$ & type \\
\hline M10 & \begin{tabular}{|c|} 
Motoyoshicho-Amagasawa, \\
Kesennuma City (Hikado Fishing Port)
\end{tabular} & 38 & 48 & 141 & 33 & 16.6 & $\mathrm{~A}$ & $\mathrm{a}$ & $\mathrm{R}$ & $\begin{array}{l}2.5 \\
3.4\end{array}$ & $\begin{array}{l}2.7 \\
3.6\end{array}$ & $\begin{array}{l}4 \\
4\end{array}$ & 3.2 & tp & 3.4 & & \\
\hline M11 & $\begin{array}{c}\text { Motoyoshicho-Maehama, Kesennuma } \\
\text { City }\end{array}$ & 38 & 48 & 141 & 33 & 19.3 & B & $\mathrm{a}$ & I & 3.9 & 4.1 & 4 & & & & 1.2 & I \\
\hline M12 & $\begin{array}{l}\text { Motoyoshicho-Toyomazawa, } \\
\text { Kesennuma City }\end{array}$ & 38 & 47 & 141 & 31 & 19.7 & A & $\mathrm{a}$ & I & 3.9 & 4.1 & 4 & & & & & \\
\hline M13 & $\begin{array}{l}\text { Motoyoshicho-Nijuichihama, } \\
\text { Kesennuma City }\end{array}$ & 38 & 46 & 141 & 31 & 17.4 & A & $\mathrm{a}$ & I & 3.1 & 3.3 & 4 & & & & & \\
\hline M14 & & 38 & 45 & 141 & 31 & 20.9 & B & $\mathrm{a}$ & $\mathrm{R}$ & & & & & & & & \\
\hline M15 & \begin{tabular}{|l|} 
Utatsu-Namiita, Minamisanriku Town \\
\end{tabular} & 38 & 45 & 141 & 32 & 19.4 & A & $\mathrm{a}$ & $\mathrm{R}$ & 2.6 & 2.8 & 4 & & & & 0.5 & I \\
\hline M16 & & 38 & 45 & 141 & 32 & 18.0 & A & $\mathrm{a}$ & I & & & & & & & & \\
\hline M17 & & 38 & 45 & 141 & 32 & 19.5 & $\mathrm{~A}$ & $\mathrm{a}$ & $\mathrm{R}$ & & & & & & & & \\
\hline M18 & \begin{tabular}{|l|} 
Utatsu-Minato, Minamisanriku Town \\
\end{tabular} & 38 & 44 & 141 & 32 & 14.5 & A & $\mathrm{a}$ & $\mathrm{R}$ & & & & & & & & \\
\hline M19 & & 38 & 44 & 141 & 32 & 14.0 & $\mathrm{~A}$ & $\mathrm{a}$ & $\mathrm{R}$ & & & & & & & & \\
\hline M20 & & 38 & 44 & 141 & 32 & 14.6 & A & $\mathrm{a}$ & I & & & & & & & & \\
\hline M21 & Utatsu-Tanoura, Minamisanriku Town & 38 & 44 & 141 & 33 & 13.1 & B & $\mathrm{a}$ & $\mathrm{R}$ & 3.0 & 3.2 & 4 & & & & 0.7 & I \\
\hline M22 & Utatsu-Kaminoyama, Minamisanriku & 38 & 44 & 141 & 33 & 14.3 & A & $\mathrm{a}$ & $\mathrm{R}$ & & & & & & & & \\
\hline M23 & Town & 38 & 44 & 141 & 33 & 16.4 & $\mathrm{~B}$ & $\mathrm{a}$ & $\mathrm{R}$ & & & & & & & & \\
\hline M28 & $\begin{array}{l}\text { Utatsu-Niranohama, Minamisanriku } \\
\text { Town }\end{array}$ & 38 & 42 & 141 & 30 & 12.9 & A & $\mathrm{a}$ & I & $\begin{array}{l}2.8 \\
3.1\end{array}$ & $\begin{array}{l}3.0 \\
3.3\end{array}$ & $\begin{array}{l}4 \\
4\end{array}$ & & & & & \\
\hline M29 & Shizugawa-Nishida, Minamisanriku & 38 & 42 & 141 & 30 & 15.1 & $\mathrm{~B}$ & $\mathrm{a}$ & $\mathrm{R}$ & 3.2 & 3.4 & 4 & & & & & \\
\hline M30 & Town & 38 & 42 & 141 & 30 & 15.0 & $\mathrm{~B}$ & $\mathrm{a}$ & $\mathrm{R}$ & 3.4 & 3.6 & 4 & & & & & \\
\hline M31 & Shizugawa-Hosoura, Minamisanriku & 38 & 42 & 141 & 30 & 13.9 & $\mathrm{~B}$ & $\mathrm{a}$ & $\mathrm{R}$ & & & & & & & & \\
\hline M32 & Town & 38 & 42 & 141 & 30 & 14.5 & A & $\mathrm{a}$ & $\mathrm{R}$ & & & & & & & & \\
\hline M33 & $\begin{array}{c}\text { Shizugawa-Omori, Minamisanriku } \\
\text { Town }\end{array}$ & 38 & 41 & 141 & 27 & 14.4 & $\mathrm{C}$ & $\mathrm{a}$ & $\mathrm{R}$ & $\begin{array}{l}4.0 \\
4.8\end{array}$ & $\begin{array}{l}4.2 \\
5.0\end{array}$ & $\begin{array}{l}4 \\
4\end{array}$ & $\mathrm{x} 4.4$ & tp & 4.6 & & \\
\hline M34 & $\begin{array}{l}\text { Shizugawa-Hayashi, Minamisanriku } \\
\text { Town }\end{array}$ & 38 & 40 & 141 & 27 & 16.0 & $\mathrm{~A}$ & $\mathrm{a}$ & I & 4.6 & 4.8 & 4 & & & & & \\
\hline M35 & Mitobe, Tokura, Minamisanriku Town & 38 & 38 & 141 & 27 & 11.7 & B & a & $\mathrm{R}$ & 4.2 & 4.4 & 4 & & & & $\begin{array}{l}1.1 \\
1.4\end{array}$ & $\begin{array}{l}\mathrm{I} \\
\mathrm{I}\end{array}$ \\
\hline M36 & $\begin{array}{l}\text { Takihama, Tokura, Minamisanriku } \\
\text { Town }\end{array}$ & 38 & 38 & 141 & 30 & 12.1 & $\mathrm{~B}$ & $\mathrm{a}$ & I & 3.2 & 3.4 & 4 & & & & & \\
\hline M37 & $\begin{array}{l}\text { Nagashizu, Tokura, Minamisanriku } \\
\text { Town }\end{array}$ & 38 & 38 & 141 & 31 & 11.3 & $\mathrm{~B}$ & $\mathrm{a}$ & $\mathrm{R}$ & 3.6 & 3.8 & 4 & & & & 0.8 & I \\
\hline M38 & $\begin{array}{l}\text { Kozashi, Kitakamicho-Jusanhama, } \\
\text { Ishinomaki City }\end{array}$ & 38 & 36 & 141 & 30 & 14.7 & A & $\mathrm{a}$ & I & $\begin{array}{l}2.8 \\
2.8\end{array}$ & $\begin{array}{l}3.0 \\
3.0\end{array}$ & $\begin{array}{l}4 \\
4\end{array}$ & & & & & \\
\hline M39 & $\begin{array}{l}\text { Aikawa, Kitakamicho-Jusanhama, } \\
\text { Ishinomaki City }\end{array}$ & 38 & 36 & 141 & 30 & 14.9 & $\mathrm{~A}$ & $\mathrm{a}$ & $\mathrm{R}$ & 3.8 & 4.0 & 4 & & & & 0.7 & I \\
\hline M40 & $\begin{array}{c}\text { Kodomari, Kitakamicho-Jusanhama, } \\
\text { Ishinomaki City }\end{array}$ & 38 & 36 & 141 & 30 & 12.0 & $\mathrm{~A}$ & $\mathrm{a}$ & $\mathrm{R}$ & 3.3 & 3.5 & 4 & & & & & \\
\hline M41 & $\begin{array}{l}\text { Omuro, Kitakamicho-Jusanhama, } \\
\text { Ishinomaki City }\end{array}$ & 38 & 36 & 141 & 30 & 14.3 & $\mathrm{C}$ & $\mathrm{a}$ & I & $\begin{array}{l}3.2 \\
4.0\end{array}$ & $\begin{array}{l}3.4 \\
4.2\end{array}$ & $\begin{array}{l}4 \\
4\end{array}$ & 3.2 & tp & 3.4 & & \\
\hline M42 & $\begin{array}{c}\text { Shirahama, Kitakamicho-Jusanhama, } \\
\text { Ishinomaki City }\end{array}$ & 38 & 35 & 141 & 28 & 15.7 & $\mathrm{~A}$ & $\mathrm{a}$ & I & 2.9 & 3.1 & 4 & & & & & \\
\hline M50 & Ogatsucho-Naburi, Ishinomaki City & 38 & 32 & 141 & 30 & 14.2 & B & $\mathrm{a}$ & $\mathrm{R}$ & $\begin{array}{l}2.6 \\
2.8 \\
\end{array}$ & $\begin{array}{l}2.8 \\
3.0\end{array}$ & $\begin{array}{l}4 \\
4 \\
\end{array}$ & 3.0 & tp & 3.2 & & \\
\hline M51 & Ogatsucho-Funakoshi, Ishinomaki City & 38 & 32 & 141 & 31 & 12.6 & B & a & $\mathrm{R}$ & 3.4 & 3.6 & 4 & & & & 0.7 & $P$ \\
\hline M53 & $\begin{array}{c}\text { Ogatsucho-Kuwanohama, Ishinomaki } \\
\text { City }\end{array}$ & 38 & 30 & 141 & 32 & 11.8 & $\mathrm{~A}$ & a & I & 3.2 & 3.4 & 4 & & & & & \\
\hline M54 & Ogatsucho-Tachihama, Ishinomaki City & 38 & 30 & 141 & 31 & 11.7 & $\mathrm{~B}$ & $\mathrm{a}$ & $\mathrm{R}$ & 2.7 & 2.9 & 4 & & & & & \\
\hline M55 & & 38 & 31 & 141 & 31 & 9.6 & $\mathrm{~B}$ & $\mathrm{a}$ & $\mathrm{R}$ & & & & & & & & \\
\hline M56 & Ogatsucho-Myojin, Ishinomaki City & 38 & 31 & 141 & 29 & 12.3 & B & $\mathrm{a}$ & $\mathrm{R}$ & $\begin{array}{l}3.3 \\
3.7\end{array}$ & $\begin{array}{l}3.5 \\
3.9\end{array}$ & $\begin{array}{l}4 \\
4\end{array}$ & 3.6 & tp & 3.8 & & \\
\hline M57 & $\begin{array}{l}\text { Funatoshinmei, Ogatsucho-Ogatsu, } \\
\text { Ishinomaki City }\end{array}$ & 38 & 31 & 141 & 28 & 15.3 & A & $\mathrm{a}$ & $\mathrm{R}$ & 4.3 & 4.5 & 4 & 4.0 & tp & 4.2 & & \\
\hline M58 & $\begin{array}{l}\text { Karakuwa, Ogatsucho-Ogatsu, } \\
\text { Ishinomaki City }\end{array}$ & 38 & 31 & 141 & 29 & 10.1 & $\mathrm{C}$ & $\mathrm{a}$ & I & 3.1 & 3.3 & 4 & & & & & \\
\hline M59 & $\begin{array}{l}\text { Wakehama, Ogatsucho-Wakehama, } \\
\text { Ishinomaki City }\end{array}$ & 38 & 30 & 141 & 29 & 13.7 & $\mathrm{~B}$ & $\mathrm{a}$ & $\mathrm{R}$ & 3.0 & 3.2 & 4 & & & & & \\
\hline M60 & $\begin{array}{l}\text { Namiita, Ogatsucho-Wakehama, } \\
\text { Ishinomaki City }\end{array}$ & 38 & 29 & 141 & 29 & 14.5 & $\mathrm{~A}$ & $\mathrm{a}$ & $\mathrm{R}$ & 3.4 & 3.6 & 4 & & & & & \\
\hline M61 & Ishihama, Onagawa Town & 38 & 27 & 141 & 28 & 16.7 & $\mathrm{~A}$ & $\mathrm{a}$ & $\mathrm{R}$ & 4.0 & 4.2 & 4 & & & & $\begin{array}{l}0.3 \\
0.4 \\
0.9 \\
1.3 \\
1.3 \\
1.5\end{array}$ & $\begin{array}{l}\text { I } \\
\text { I } \\
\text { I } \\
\text { I } \\
\text { I } \\
\text { R }\end{array}$ \\
\hline M62 & Oishiharahama, Onagawa Town & 38 & 24 & 141 & 28 & 13.0 & $\mathrm{~B}$ & $\mathrm{a}$ & $R$ & 4.4 & 4.6 & 4 & 2.9 & tp & 3.1 & $\begin{array}{l}1.4 \\
1.4 \\
1.5\end{array}$ & $\begin{array}{l}\text { I } \\
\text { I } \\
\text { I }\end{array}$ \\
\hline M65 & Samenoura, Ishinomaki City & 38 & 23 & 141 & 29 & 17.9 & $\mathrm{C}$ & $\mathrm{a}$ & I & 4.5 & 4.7 & 4 & & & & & \\
\hline
\end{tabular}


Table 2

continued

\begin{tabular}{|c|c|c|c|c|c|c|c|c|c|c|c|c|c|c|c|c|c|}
\hline \multirow[t]{2}{*}{ No. } & \multirow[t]{2}{*}{ Location Name } & \multicolumn{2}{|c|}{ Latitude } & \multicolumn{2}{|c|}{ Longitude } & \multirow{2}{*}{$\begin{array}{c}\begin{array}{c}2011 \\
\text { height }^{\mathrm{a}}\end{array} \\
(\mathrm{m})\end{array}$} & \multirow[t]{2}{*}{ rel. $^{\text {b }}$} & \multirow[t]{2}{*}{ acc. $^{c}$} & \multirow[t]{2}{*}{ type } & \multicolumn{6}{|c|}{1960 height } & \multicolumn{2}{|c|}{2010 height } \\
\hline & & deg & $\min$ & $\operatorname{deg}$ & $\min$ & & & & & $\begin{array}{r}\mathrm{CFI}^{\mathrm{d}} \\
(\mathrm{m})\end{array}$ & 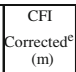 & $\begin{array}{l}\text { CFI } \\
\text { acc. }^{f}\end{array}$ & $\begin{array}{c}\mathrm{JMA}^{\mathrm{g}} \\
(\mathrm{m})\end{array}$ & \begin{tabular}{r|r} 
JMA \\
datum
\end{tabular} & \begin{tabular}{|c} 
JMA \\
$\begin{array}{c}\text { Correctede }^{\mathrm{e}} \\
(\mathrm{m})\end{array}$
\end{tabular} & $\underset{(\mathrm{m})}{\text { Tsuji/Imai }}{ }^{\mathrm{h}}$ & type \\
\hline M66 & Oyagawahama, Ishinomaki City & 38 & 22 & 141 & 29 & 17.8 & B & $\mathrm{a}$ & $\mathrm{R}$ & 5.0 & 5.2 & 2 & 5.4 & tp & 5.6 & & \\
\hline M67 & \multirow[t]{2}{*}{ Yagawahama, Ishinomaki City } & 38 & 22 & 141 & 29 & 18.7 & $\mathrm{C}$ & $\mathrm{a}$ & I & 4.0 & 4.2 & 4 & & & & & \\
\hline M68 & & 38 & 22 & 141 & 29 & 21.2 & B & $\mathrm{a}$ & $\bar{R}$ & $\begin{array}{l}4.6 \\
4.7\end{array}$ & $\begin{array}{l}4.8 \\
4.9\end{array}$ & $\begin{array}{l}4 \\
4\end{array}$ & & & & & \\
\hline M69 & \multirow[t]{2}{*}{ Tomarihama, Ishinomaki City } & 38 & 22 & 141 & 31 & 12.4 & A & $\mathrm{a}$ & $\mathrm{R}$ & \multirow[t]{2}{*}{3.2} & \multirow[t]{2}{*}{3.4} & \multirow[t]{2}{*}{4} & & & & 0.8 & $T$ \\
\hline M70 & & 38 & 22 & 141 & 31 & 12.2 & A & $\mathrm{a}$ & $\mathrm{R}$ & & & & & & & & \\
\hline M71 & Kugunarihama, Ishinomaki City & 38 & 19 & 141 & 30 & 6.3 & A & $\mathrm{a}$ & I & $\begin{array}{l}3.6 \\
3.8\end{array}$ & $\begin{array}{l}3.8 \\
4.0\end{array}$ & $\begin{array}{l}4 \\
2\end{array}$ & & & & & \\
\hline M72 & Koamikurahama, Ishinomaki City & 38 & 21 & 141 & 27 & 12.9 & $\mathrm{~B}$ & $\mathrm{a}$ & $\mathrm{R}$ & $\begin{array}{l}3.2 \\
3.8\end{array}$ & $\begin{array}{l}3.4 \\
4.0\end{array}$ & $\begin{array}{l}4 \\
4\end{array}$ & & & & & \\
\hline M73 & Fukkiura, Ishinomaki City & 38 & 21 & 141 & 27 & 8.2 & B & $\mathrm{a}$ & $\mathrm{R}$ & 3.4 & 3.6 & 4 & & & & & \\
\hline M75 & Kozumihama, Ishinomaki City & 38 & 22 & 141 & 27 & 8.5 & B & $\mathrm{a}$ & I & 5.0 & 5.2 & 4 & $\begin{array}{c}4.4 \\
\times 5.0 \\
\end{array}$ & $\begin{array}{l}\text { tp } \\
\text { tp }\end{array}$ & $\begin{array}{l}4.6 \\
5.2\end{array}$ & & \\
\hline M76 & Momonoura, Ishinomaki City & 38 & 24 & 141 & 26 & 12.5 & $\mathrm{~B}$ & $\mathrm{a}$ & $\mathrm{R}$ & $\begin{array}{l}4.1 \\
4.7 \\
\end{array}$ & $\begin{array}{l}4.3 \\
4.9 \\
\end{array}$ & 4 & $\begin{array}{c}5.0 \\
\times 5.2 \\
\end{array}$ & $\begin{array}{l}\text { tp } \\
\text { tp }\end{array}$ & $\begin{array}{l}5.2 \\
5.4\end{array}$ & & \\
\hline B1 & \multirow{5}{*}{$\begin{array}{l}\text { Hirakatacho, } \\
\text { Kitaibaraki City }\end{array}$} & 36 & 51 & 140 & 48 & 6.6 & A & $\mathrm{a}$ & I & & & & \multirow[t]{5}{*}{5} & \multirow[t]{5}{*}{ da } & \multirow[t]{5}{*}{2.5} & & \\
\hline $\mathrm{B} 2$ & & 36 & 51 & 140 & 48 & 7.1 & A & $\mathrm{a}$ & I & & & & & & & & \\
\hline B3 & & 36 & 51 & 140 & 48 & 7.2 & A & $\mathrm{a}$ & I & & & & & & & & \\
\hline B4 & & 36 & 51 & 140 & 48 & 8.1 & B & $\mathrm{b}$ & $\mathrm{R}$ & & & & & & & & \\
\hline B5 & & 36 & 51 & 140 & 48 & 7.9 & B & b & $\mathrm{R}$ & & & & & & & & \\
\hline B6 & $\begin{array}{l}\text { Otsucho, Kitaibaraki City } \\
\text { (Otsu Fishing Port) }\end{array}$ & 36 & 50 & 140 & 47 & 4.9 & A & $\mathrm{a}$ & I & 1.5 & 1.4 & 3 & 5.7 & da & 2.9 & 1.0 & I \\
\hline B19 & $\begin{array}{l}\text { Osecho, Hitachi City } \\
\text { (Ose Fishing Port) }\end{array}$ & 36 & 35 & 140 & 39 & 5.1 & A & $\mathrm{a}$ & I & & & & 3 & $\begin{array}{ll}\mathrm{msl} \\
\mathrm{msl}\end{array}$ & 3.0 & $\begin{array}{l}0.8 \\
1.3 \\
1.5 \\
1.5 \\
1.5 \\
1.5 \\
1.6 \\
1.8 \\
1.8\end{array}$ & $\begin{array}{l}P \\
R \\
R \\
R \\
R \\
R \\
R \\
R \\
R\end{array}$ \\
\hline B24 & $\begin{array}{l}\text { Kujicho, Hitachi City } \\
\text { (Kuji Fishing Port) }\end{array}$ & 36 & 30 & 140 & 38 & 4.1 & B & $\mathrm{b}$ & $\mathrm{R}$ & 2.3 & 2.2 & 3 & 3 & un & & & \\
\hline B27 & $\begin{array}{l}\text { Isozakicho, Hitachinaka City } \\
\text { (Isozaki Fishing Port) }\end{array}$ & 36 & 23 & 140 & 37 & 3.5 & A & $\mathrm{a}$ & I & & & & 3 & $\mathrm{da}$ & 1.5 & & \\
\hline B28 & $\begin{array}{l}\text { Hiraisocho, Hitachinaka City } \\
\text { (Hiraiso Fishing Port) }\end{array}$ & 36 & 21 & 140 & 37 & 4.2 & $\mathrm{~A}$ & $\mathrm{a}$ & I & & & & 2.5 & $\mathrm{da}$ & 1.3 & & \\
\hline B29 & $\begin{array}{l}\text { Kaimoncho, Hitachinaka City } \\
\text { (Nakaminato Fishing Port) }\end{array}$ & 36 & 20 & 140 & 36 & 3.2 & A & $\mathrm{a}$ & I & $\begin{array}{l}1.25 \\
2.1\end{array}$ & $\begin{array}{l}1.41 \\
2.2\end{array}$ & $\begin{array}{l}1 \\
5\end{array}$ & 2 & $\mathrm{da}$ & 1.0 & & \\
\hline B30 & $\begin{array}{c}\text { Minatochuo, Oarai Town } \\
\text { (Ibaraki Port, Oarai Port District) }\end{array}$ & 36 & 19 & 140 & 34 & 4.5 & $\mathrm{~A}$ & $\mathrm{a}$ & I & & & & & & & $\begin{array}{l}1.2 \\
1.2\end{array}$ & $\begin{array}{l}\mathrm{P} \\
\mathrm{P}\end{array}$ \\
\hline B31 & & 36 & 19 & 140 & 34 & 4.6 & A & $\mathrm{a}$ & I & & & & & & & & \\
\hline B32 & Takeigama, Kashima City & 36 & 4 & 140 & 37 & 3.5 & A & b & I & & & & 2 & un & & 1.5 & $\mathrm{R}$ \\
\hline B33 & & 36 & 4 & 140 & 37 & 3.7 & A & b & $\mathrm{R}$ & & & & & & & & \\
\hline B34 & Hamatsuga, Kashima City & 36 & 4 & 140 & 37 & 3.8 & B & b & I & & & & & & & & \\
\hline B35 & $\begin{array}{l}\text { Higashifukashiba, Kamisu City } \\
\text { (Kashima Port) }\end{array}$ & 35 & 55 & 140 & 40 & 5.7 & $\mathrm{~B}$ & $\mathrm{a}$ & $\mathrm{R}$ & & & & & & & $\begin{array}{l}0.8 \\
1.6 \\
\end{array}$ & $\begin{array}{l}\mathrm{T} \\
\mathrm{I}\end{array}$ \\
\hline B36 & $\begin{array}{l}\text { Hasakishinko, Kamisu City (Shin } \\
\text { Fishing Port) }\end{array}$ & 35 & 45 & 140 & 51 & 3.3 & A & $\mathrm{a}$ & I & & & & & & & $\begin{array}{l}0.4 \\
0.5\end{array}$ & $\begin{array}{l}P \\
P\end{array}$ \\
\hline $\mathrm{C} 1$ & $\begin{array}{l}\text { Araoicho, Choshi City (Choshi Fishing } \\
\text { Port) }\end{array}$ & 35 & 44 & 140 & 50 & 2.7 & A & $\mathrm{a}$ & I & $\begin{array}{c}0.6 \\
0.95\end{array}$ & $\begin{array}{l}0.7 \\
1.05\end{array}$ & $\begin{array}{l}5 \\
5\end{array}$ & & & & $\begin{array}{l}0.5 \\
0.5 \\
0.6\end{array}$ & $\begin{array}{l}\mathrm{P} \\
\mathrm{P} \\
\mathrm{T}\end{array}$ \\
\hline $\mathrm{C} 2$ & $\begin{array}{l}\text { Inuwaka, Choshi City (Inuwaka } \\
\text { Fishing Port) }\end{array}$ & 35 & 42 & 140 & 51 & 4.8 & $\mathrm{~A}$ & $\mathrm{a}$ & I & 2.09 & 2.19 & 5 & 2.14 & \begin{tabular}{c|} 
msl \\
(Togawa)
\end{tabular} & 2.2 & $\begin{array}{l}1.0 \\
1.2\end{array}$ & $\begin{array}{l}\text { I } \\
\text { I }\end{array}$ \\
\hline $\mathrm{C} 3$ & $\begin{array}{r}\begin{array}{c}\text { Shimonagai, Asahi City } \\
\text { Fishing Port) }\end{array} \\
\text { (lioka }\end{array}$ & 35 & 42 & 140 & 44 & 3.2 & $\mathrm{~A}$ & $\mathrm{a}$ & I & & & & 3.5 & tp & 3.5 & $\begin{array}{l}0.9 \\
1.5\end{array}$ & $\begin{array}{l}\mathrm{R} \\
\mathrm{R}\end{array}$ \\
\hline $\mathrm{C} 4$ & Hiramatsu, Asahi City & 35 & 42 & 140 & 43 & 6.3 & A & $\mathrm{a}$ & I & 3.7 & 3.8 & 2 & & & & & \\
\hline $\mathrm{C} 5$ & Ashikawa, Asahi City & 35 & 42 & 140 & 40 & 5.1 & A & $\mathrm{a}$ & I & & & & & & & 0.3 & $\mathrm{P}$ \\
\hline C6 & & 35 & 42 & 140 & 40 & 7.9 & B & $\mathrm{a}$ & $\mathrm{R}$ & & & & & & & & \\
\hline $\mathrm{C} 15$ & $\begin{array}{l}\text { Koseki, Kujukuri Town (Katakai } \\
\text { Fishing Port) }\end{array}$ & 35 & 32 & 140 & 27 & 2.5 & $\mathrm{~A}$ & $a$ & I & 1.4 & 1.5 & 3 & & & & $\begin{array}{l}0.6 \\
0.7 \\
0.7 \\
0.7 \\
0.7\end{array}$ & $\begin{array}{c}\mathrm{R} \\
\mathrm{R} \\
\mathrm{R} \\
\mathrm{R} \\
\mathrm{I}\end{array}$ \\
\hline $\mathrm{C} 18$ & Sendokyu, Ichinomiya Town & 35 & 23 & 140 & 23 & 2.9 & A & $\mathrm{c}$ & II & 1.0 & 1.1 & 3 & 2 & nt & 2.0 & & \\
\hline $\mathrm{C} 22$ & Ohara, Isumi City (Ohara Fishing Port) & 35 & 15 & 140 & 24 & 2.6 & B & $\mathrm{a}$ & I & $\begin{array}{l}1.8 \\
1.8\end{array}$ & $\begin{array}{l}1.6 \\
1.6\end{array}$ & $\begin{array}{l}1 \\
1\end{array}$ & $2-3$ & un & & 0.8 & I \\
\hline C26 & $\begin{array}{c}\text { Hama, Onjuku Town (Onjuku Fishing } \\
\text { Port) }\end{array}$ & 35 & 11 & 140 & 21 & 2.3 & A & $\mathrm{a}$ & I & 1.7 & 1.5 & 3 & & & & $\begin{array}{l}0.5 \\
0.7 \\
1.1\end{array}$ & $\begin{array}{l}R \\
P \\
R\end{array}$ \\
\hline $\mathrm{C} 27$ & $\begin{array}{l}\text { Hamakatsuura, Katsuura City } \\
\text { (Katsuura Fishing Port) }\end{array}$ & 35 & 9 & 140 & 19 & 2.0 & $\mathrm{~B}$ & $\mathrm{a}$ & $\mathrm{R}$ & $\begin{array}{l}1.9 \\
2.2\end{array}$ & $\begin{array}{l}1.7 \\
2.0\end{array}$ & $\begin{array}{l}3 \\
2\end{array}$ & $2.0-2.5$ & $\mathrm{msl}$ & $1.8-2.3$ & $\begin{array}{l}0.6 \\
0.8\end{array}$ & $\begin{array}{l}\mathrm{P} \\
\mathrm{P}\end{array}$ \\
\hline
\end{tabular}


Table 2

continued

\begin{tabular}{|c|c|c|c|c|c|c|c|c|c|c|c|c|c|c|c|c|c|}
\hline \multirow[t]{2}{*}{ No. } & \multirow[t]{2}{*}{ Location Name } & \multicolumn{2}{|c|}{ Latitude } & \multicolumn{2}{|c|}{ Longitude } & \multirow{2}{*}{$\begin{array}{c}\begin{array}{c}2011 \\
\text { height }^{\mathrm{a}}\end{array} \\
(\mathrm{m})\end{array}$} & \multirow[t]{2}{*}{ rel. $^{\text {b }}$} & \multirow[t]{2}{*}{ acc. $^{\mathrm{c}}$} & \multirow[t]{2}{*}{ type } & \multicolumn{6}{|c|}{1960 height } & \multicolumn{2}{|c|}{2010 height } \\
\hline & & deg & $\min$ & deg & $\min$ & & & & & $\begin{array}{r}\mathrm{CFI}^{\mathrm{d}} \\
(\mathrm{m})\end{array}$ & \begin{tabular}{|c|} 
CFI \\
Corrected $^{\mathrm{e}}$ \\
$(\mathrm{m})$
\end{tabular} & $\begin{aligned} \text { CFI } \\
\text { acc. }^{f}\end{aligned}$ & $\begin{array}{c}\mathrm{JMA}^{\mathrm{g}} \\
(\mathrm{m})\end{array}$ & $\begin{array}{r}\text { JMA } \\
\text { datum }\end{array}$ & \begin{tabular}{|c|} 
JMA \\
Corrected $^{\mathrm{e}}$ \\
$(\mathrm{m})$
\end{tabular} & $\begin{array}{c}\text { Tsuji/Imai } \\
(\mathrm{m})\end{array}$ & type \\
\hline $\mathrm{C} 29$ & $\begin{array}{c}\text { Kominato, Kamogawa City (Kominato } \\
\text { Fishing Port) }\end{array}$ & 35 & 7 & 140 & 12 & 1.7 & B & $\mathrm{a}$ & $P$ & 1.5 & 1.3 & 3 & & & & & \\
\hline $\mathrm{C} 30$ & $\begin{array}{l}\text { Amatsu, Kamogawa City (Amatsu } \\
\text { Fishing Port) }\end{array}$ & 35 & 7 & 140 & 10 & 1.8 & B & $\mathrm{a}$ & $\mathrm{R}$ & 1.3 & 1.1 & 2 & & & & & \\
\hline $\mathrm{C} 31$ & & 35 & 7 & 140 & 10 & 1.5 & B & $\mathrm{a}$ & $\mathrm{R}$ & & & & & & & & \\
\hline C34 & $\begin{array}{l}\text { Wadacho-Wada, Minamiboso City } \\
\text { (Wada Fishing Port) }\end{array}$ & 35 & 2 & 140 & 1 & 1.9 & $\mathrm{~A}$ & $\mathrm{a}$ & I & 1.0 & 0.8 & 2 & 0.7 & ht & 0.9 & & \\
\hline C37 & $\begin{array}{c}\text { Chikuracho-Hedate, Minamiboso City } \\
\text { (Chikura Fishing Port) }\end{array}$ & 34 & 57 & 139 & 58 & 1.4 & $\mathrm{~B}$ & $\mathrm{a}$ & I & $\begin{array}{l}1.3 \\
1.2\end{array}$ & $\begin{array}{l}1.0 \\
1.1\end{array}$ & $\begin{array}{l}3 \\
3\end{array}$ & 0.9 & ht & 1.1 & & \\
\hline $\mathrm{C} 38$ & & 34 & 57 & 139 & 58 & 0.9 & B & $\mathrm{a}$ & $P$ & & & & & & & & \\
\hline C39 & $\begin{array}{c}\text { Shirahamacho-Otohama, Minamiboso } \\
\text { City (Otohama Fishing Port) }\end{array}$ & 34 & 55 & 139 & 56 & 1.2 & B & $\mathrm{a}$ & $\mathrm{R}$ & 1.3 & 1.1 & 3 & & & & & \\
\hline $\mathrm{C} 40$ & $\begin{array}{c}\text { Shirahamacho-Shirahama, Minamiboso } \\
\text { City (Nojimahigashi Fishing Port) }\end{array}$ & 34 & 54 & 139 & 53 & 1.0 & B & $\mathrm{a}$ & $\mathrm{R}$ & 1.0 & 0.8 & $3 \mathrm{i}$ & 2.0 & da & 1.0 & & \\
\hline $\mathrm{C} 43$ & $\begin{array}{l}\text { Mera, Tateyama City (Tomisaki } \\
\text { Fishing Port) }\end{array}$ & 34 & 55 & 139 & 50 & 2.0 & $\mathrm{~A}$ & $\mathrm{a}$ & I & 1.32 & 1.11 & 5 & 1.73 & nt & 1.7 & $\begin{array}{l}0.7 \\
0.8\end{array}$ & $\begin{array}{l}\mathrm{P} \\
\mathrm{T}\end{array}$ \\
\hline
\end{tabular}

$R$ runup height, $I$ inundation height, $P$ tsunami height in ports, $T$ tide gauge, $d a$ double amplitude, $m s l$ tsunami heights above mean sea level, $d l$ datum line, $t p$ tsunami heights above TP, $n t$ tsunami heights above sea level at time of maximum tsunami, $h t$ tsunami heights above high tide level of the tsunami arrival date, un unknown, $x$ eyewitness accounts

a 2011 heights above sea level at time of maximum tsunami

b Rel.: reliability, A: most reliable based on clear physical evidence or eyewitness account; B: mostly based on natural traces; C: least reliable based on equivocal evidence

c Acc.: accuracy; a: measurement error $<0.2 \mathrm{~m}$; b $0.2 \leq$ error $\leq 0.5 \mathrm{~m}$; c error $>0.5 \mathrm{~m}$

d Tsunami heights above Tokyo Peil (TP) taken from the Committee of Field Investigation of the Chilean Tsunami of 1960 (CFI 1961). The numbers in parentheses indicate questionable data

e Tsunami heights above sea level at time of maximum tsunami

f 5 : Values observed by tide gauges; 4: values with highest accuracy; 3 : values with moderate accuracy; 2: values with fair accuracy; 1 : values obtained by other sources

g Tsunami heights from JMA (1961)

h Tsunami heights above sea level at the time of maximum tsunami taken from TsuJ et al. (2010) or Imai et al. (2010)

i Nojimanishi

heights were measured as 17 and $21 \mathrm{~m}$ (I133-I134). The 1960 tsunami heights were 3 and $4 \mathrm{~m}$ by CFI (1961), and 4 and $5 \mathrm{~m}$ by the JMA (1961), respectively.

\subsection{Northern and Southern Sanriku Coasts}

Along the northern Sanriku coast (north of latitude $40.2^{\circ} \mathrm{N}$ ), the 2011 tsunami heights drastically decrease toward the north from 20 to $5 \mathrm{~m}$. The two preceding Sanriku tsunami heights also become smaller toward the north (Fig. 14). The 1896 heights are similar to the 2011 heights (median ratio is 1.01; Table 3), while the 1933 heights are smaller (median ratio 0.66). The 1960 and 2010 Chilean tsunami heights are more uniform throughout the Sanriku coast, and are smaller than the three Sanriku tsunamis (median ratio of 1960/2011 heights is 0.42 , while one 2010 height is 0.15 of the 2011 height).

Along the southern Sanriku coast (south of latitudes $39.0^{\circ} \mathrm{N}$ ), the 2011 tsunami heights range mostly from 10 to $20 \mathrm{~m}$, and larger than all the previous tsunamis (median ratio is $0.29,0.24,0.28$, 0.06 for 1896, 1933, 1960, and 2010 tsunami, respectively). The 1960 and 2010 tsunami heights are more uniformly distributed. The 1896 and 1933 tsunami heights drastically decrease toward the south 


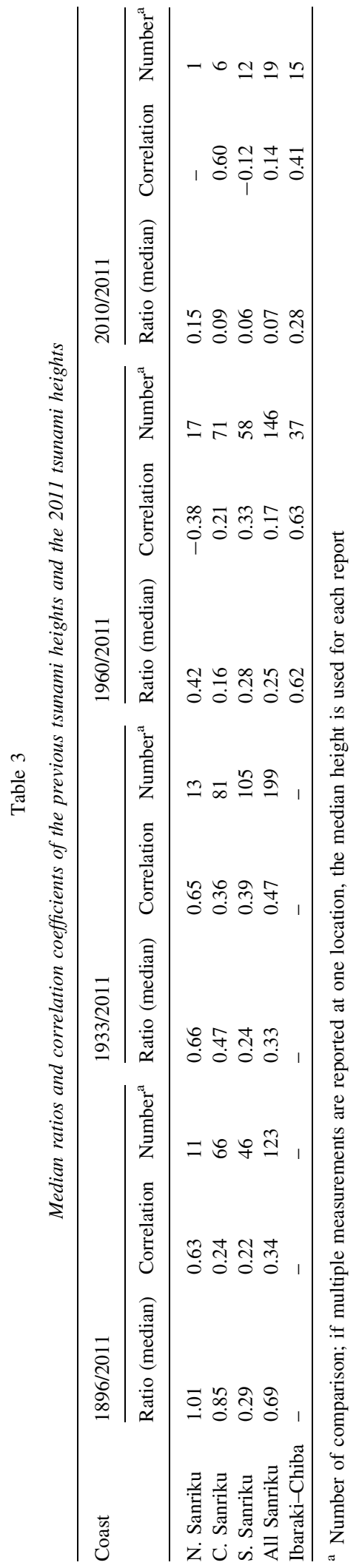

and become smaller than the 1960 Chilean tsunami heights at the southernmost Sanriku coast.

At Utatsu-Tanoura in Minamisanriku Town, the 2011 tsunami was evidently much higher than the other historical tsunamis. Almost all the houses in low-lying areas were washed away, and large amounts of rubble, fishing boats, and equipment were transported by the tsunami. The 2011 runup heights were between 13 and $16 \mathrm{~m}$ (M21-M23), while the 1896 tsunami heights were 16 feet ( $5 \mathrm{~m}$; IKI 1897) or 4-11 m (Matsuo 1933). The 1933 tsunami heights were 5-6 m (Matsuo 1933; Earthquake Research Institute 1934). The 1960 tsunami height was $3 \mathrm{~m}$ by CFI (1961) and the 2010 height was $1 \mathrm{~m}$ by Tsus et al. (2010), respectively.

At Ogatsucho-Wakehama in Ishinomaki City, the 2011 tsunami height was the largest, followed by the 1960 Chilean tsunami height, which was larger than the 1896 and 1933 Sanriku tsunamis. The 2011 tsunami inundated areas up to a temple $\sim 250 \mathrm{~m}$ from the coast, and almost all houses were swept away. The 2011 runup height was $14 \mathrm{~m}$ (M59), while the reported 1896 and 1933 tsunami heights were $\sim 2 \mathrm{~m}$ and the 1960 tsunami height was reported as $3 \mathrm{~m}$ by CFI (1961).

\subsection{Correlation of Tsunami Heights}

The tsunami heights from the 1896 and 1933 Sanriku tsunamis and the 1960 and 2010 Chilean tsunamis were compared with the 2011 tsunami heights at the same locations (Fig. 19; Table 3). Along the Sanriku coasts, the 2011 tsunami heights are positively correlated with those from the 1896 and 1933 Sanriku tsunamis. The correlation coefficient of the 2011 and 1896 tsunamis is 0.34 , and that for the 2011 and 1933 tsunamis is 0.47 for the entire Sanriku coasts. The correlation coefficients are much larger on the northern Sanriku coast (0.63 and 0.65 for 2011-1896 and 2011-1933, respectively), but smaller on the central coast $(0.24$ and 0.36$)$ and southern coast ( 0.22 and 0.39 ). The positive correlation coefficients indicate that the tsunami height variation is similar for local tsunamis. On the other hand, the 2011 tsunami heights are weakly correlated with those from the Chilean tsunami heights; the correlation coefficients are 0.17 for the 2011 and 


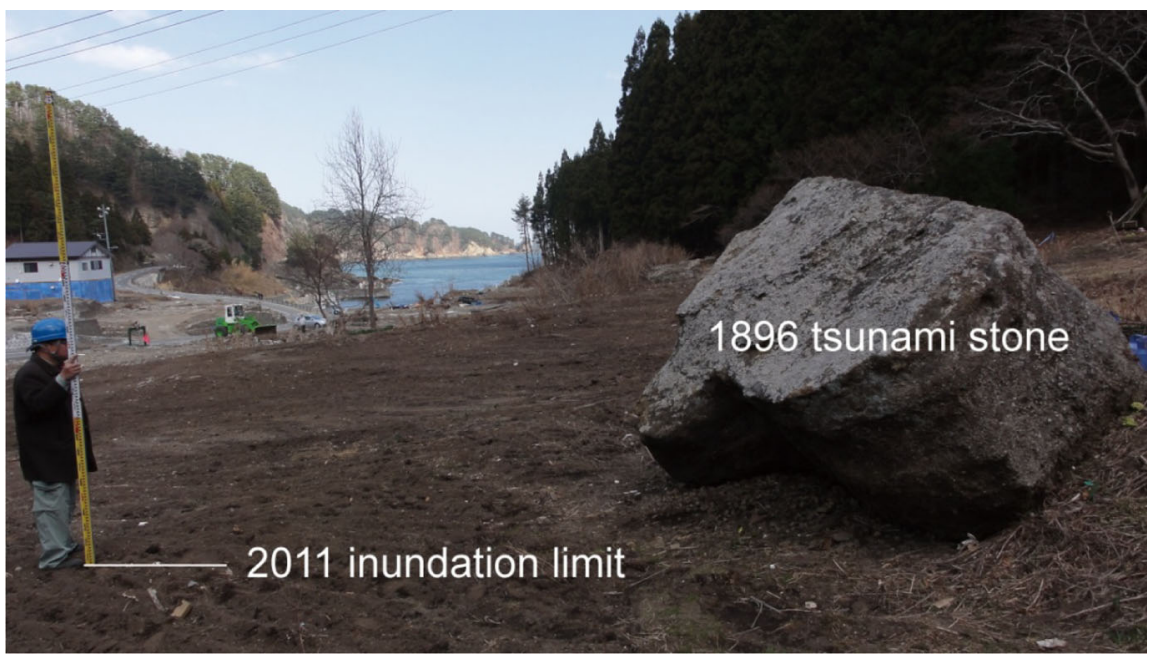

Figure 15

Tsunami stone transported by the 1896 Sanriku tsunami at Raga, Tanohata Village, Iwate Prefecture. The 2011 tsunami inundation limit (24.5 $\mathrm{m}$; I37) is just below this stone

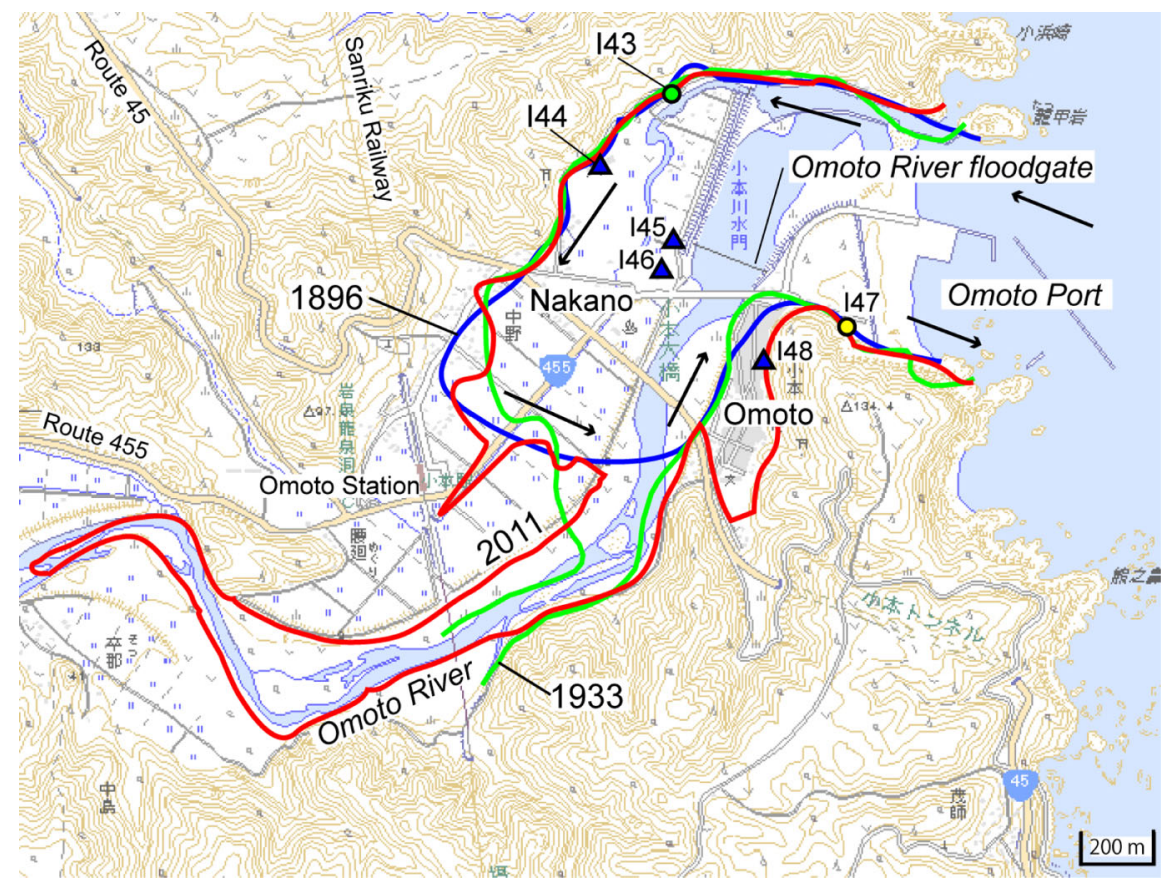

Figure 16

Inundation areas from the 1896, 1933, and 2011 tsunamis at Omoto, Iwaizumi Town, Iwate prefecture. The blue curve indicates the area severely damaged by the 1896 tsunami from IKI (1897). The black arrows show the direction of the 1896 tsunami reported by the Fudai Village chief in those days. The green curves show the inundation limits of the 1933 tsunami from the EARTHQUAKE RESEARCH InSTITUTE (1934). The red curve indicates the inundation limit of the 2011 tsunami by HaRaguchi and Iwamatsu (2011). The circles and triangles indicate the measurement points of runup and inundation heights, respectively, with the same color code as Fig. 3

1960 tsunamis and 0.14 for the 2011 and 2010 tsunamis for the entire Sanriku coastline. They are negatively correlated on the northern Sanriku coast for the 1960 tsunami and on the southern coast for the 2010 tsunami. These indicate that tsunami height distribution of local tsunamis is different from that of trans-Pacific tsunamis. 


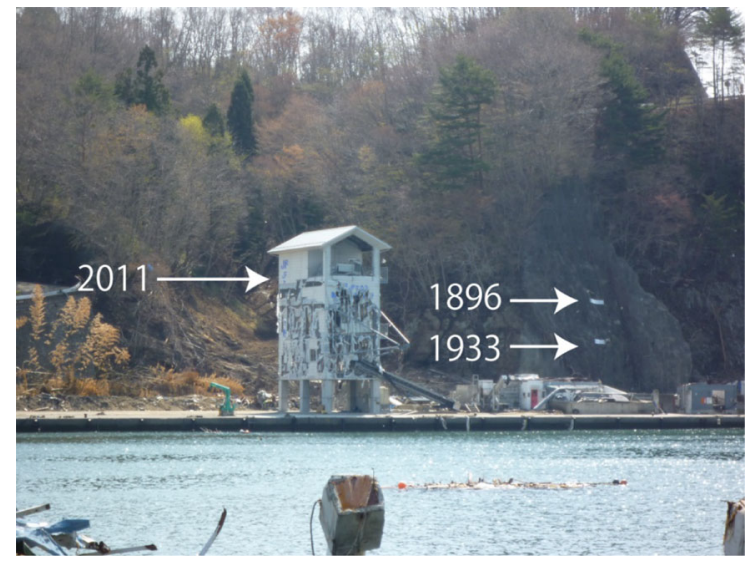

Figure 17

Icehouse of the Japan Fisheries Cooperatives at Taro Fishing Port, which was severely damaged by the 2011 tsunami (14.8 m; I72). The two white markers on the back cliff show the heights of the 1896 (14.6 m; IKI 1897) and 1933 (10 m; Earthluake Research InstituTE 1934) Sanriku tsunamis. The tsunami height of $10 \mathrm{~m}$ for the 1933 tsunami was measured at a slightly different location; the tsunami heights at this location measured by EARTHQUAKE RESEARCH Institute (1934) and Matsuo (1933) were 7.0 and $6.4 \mathrm{~m}$, respectively

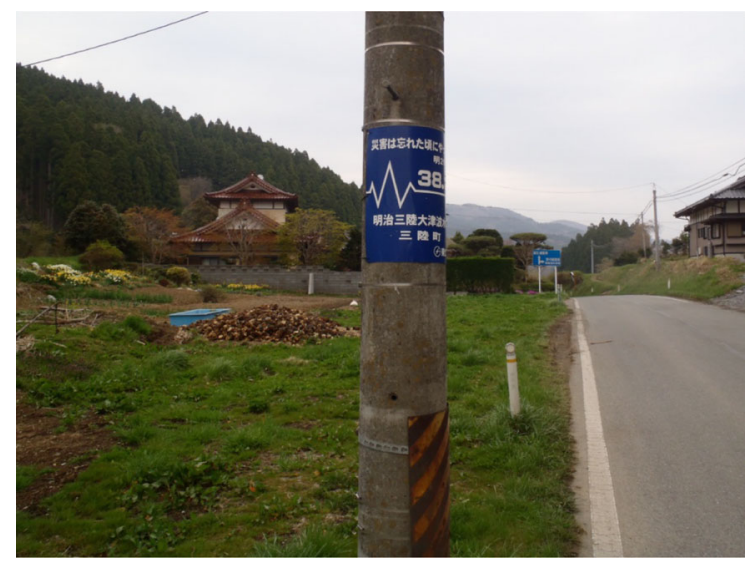

Figure 18

Maximum tsunami height of $38.2 \mathrm{~m}$ from the 1896 Sanriku tsunami shown on a pole near the border of Okubo and Shirahama, Sanrikucho-Ryori, Ofunato City, Iwate Prefecture (labeled Ryori in Fig. 14). The 2011 tsunami with heights of $16.8 \mathrm{~m}$ (I133) and $21.2 \mathrm{~m}$ (I134) did not reach this location

\subsection{Controlling Factors of Tsunami Heights and their Variations}

The type, location, and magnitude of the 1896, 1933, and 2011 earthquakes are all different. The 1896 Sanriku earthquake $\left(M_{\mathrm{s}} 7.2\right)$ was an example of a "tsunami earthquake" (KANAMORI 1972) that produces weak ground shaking, but a very large tsunami. The 1896 tsunami source was estimated to be near the trench axis with a $210 \mathrm{~km}$ fault length and $\sim 50 \mathrm{~km}$ fault width (Fig. 1; TANIOKA and Satake 1996; Tanioka and Seno 2001). The seafloor deformation, landward subsidence, and seaward uplift were limited near the trench axis (Fig. 20). The 1933 Sanriku earthquake $\left(M_{\mathrm{s}} 8.5\right)$ was an outerrise earthquake with a normal faulting mechanism (KANAMORI 1971). The seafloor deformation was dominantly subsidence (AIDA 1977). The 2011 Tohoku earthquake was the largest $\left(M_{\mathrm{w}} 9.0\right)$. The seafloor deformation extended much further than the above-mentioned Sanriku earthquakes. The largest slip occurred at around $38.3^{\circ} \mathrm{N}, 143.3^{\circ} \mathrm{E}$, to the east of the epicenter, but the maximum tsunami heights were recorded on the central Sanriku coast $\sim 100 \mathrm{~km}$ north of the largest slip. The 2011 Tohoku earthquake can be considered a combination of a great interplate earthquake and a "tsunami earthquake" (Fig. 1; Fusi et al. 2011; SATAKE et al. 2013), while OKaL (2013) argued that there is no evidence of 'slowness' in the earthquake source, and Grilli et al. (2013) suggested additional tsunami generation mechanisms not represented in the coseismic sources (e.g., splay faults, sub-marine mass failure).

The tsunami heights from the three Sanriku tsunamis exhibit a large variation on the central Sanriku coast (between $39.0^{\circ}$ and $40.2^{\circ} \mathrm{N}$ ) regardless of the type, location, and magnitude of the earthquakes. The tsunami heights from the 1896 and 2011 earthquakes ranging 5-40 $\mathrm{m}$ are approximately similar on the central Sanriku coast, whereas the seismologically determined earthquake magnitudes of $M_{\mathrm{s}} 7.2$ for the 1896 were much smaller than the 2011 earthquake $\left(M_{\mathrm{w}} 9.0\right)$. While the source region of the 2011 earthquake includes the rupture area of the 1896 earthquake, the 1896 tsunami heights are higher than the 2011 tsunami heights at some locations. These facts demonstrate that the coastal tsunami heights on the central Sanriku coast are not necessarily controlled by the location, type, or the magnitude of earthquake, and that the huge tsunami was not a surprise.

The Sanriku coast consists of numerous bays of various sizes and depths; it is called a ria coast, as it 

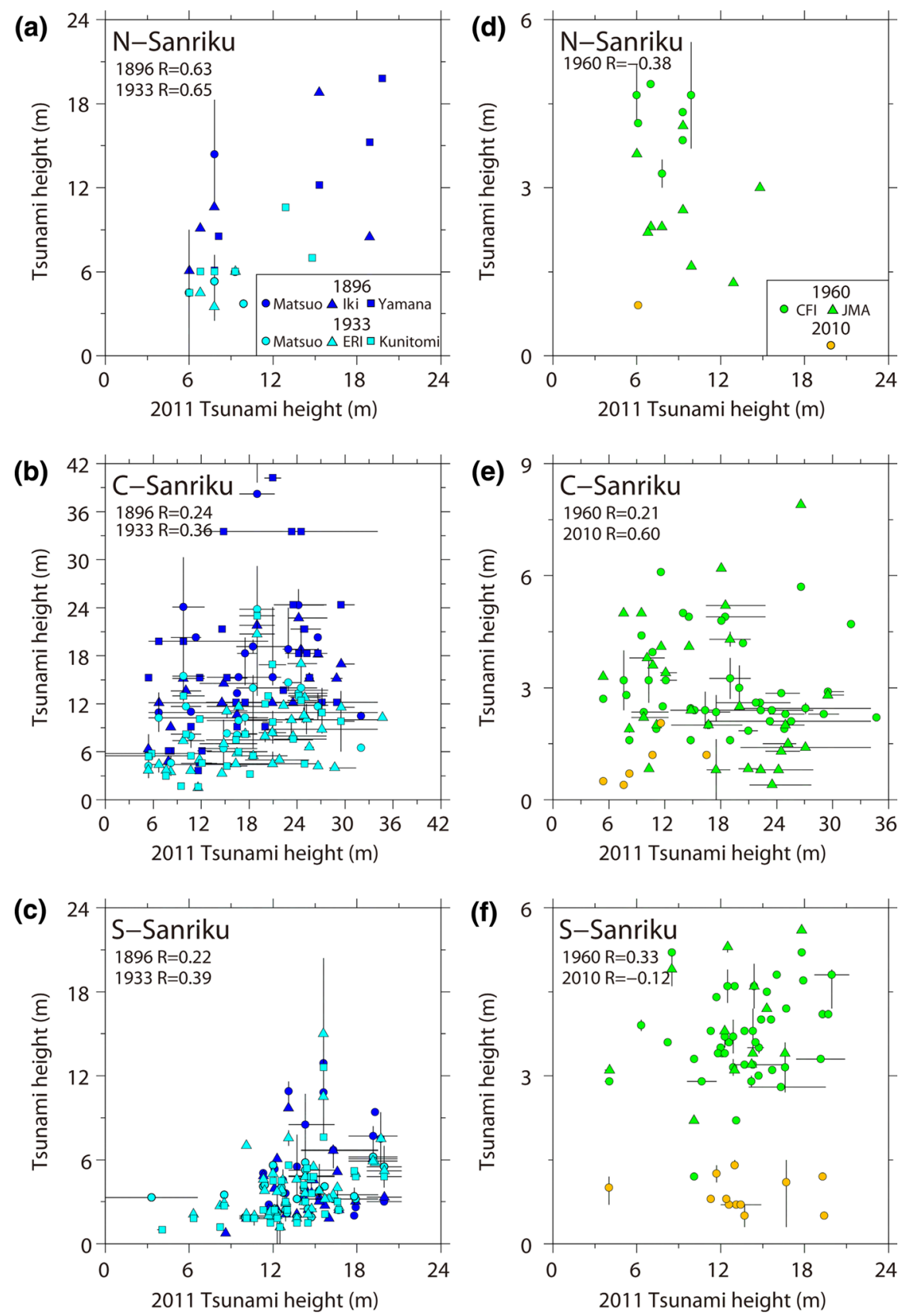

Figure 19

Comparison between the 2011 tsunami and the 1896 and 1933 Sanriku tsunamis for the northern (a), central (b), and southern Sanriku coasts (c). The similar comparison between the 2011 tsunami and the 1960 and 2010 Chilean tsunamis are shown in d-f. Orange circles indicate the 2010 tsunami heights from Tsus et al. (2010). Other symbols are the same as in Fig. 14. Multiple height data at the same locations are represented as median values with ranges (shown as bars). The correlation coefficients of the previous tsunami heights and the 2011 tsunami heights are also shown

was created by the submergence and subsequent flooding of mountainous terrain (е.g., ОтUкA 1934; KoIKe et al. 2005). The characteristic periods of sea level oscillations in bays are also variable; $55.2 \mathrm{~min}$ for Miyako Bay, the largest bay on the Sanriku coast, while $27.0 \mathrm{~min}$ for Otsuchi (Honde et al. 1908). 


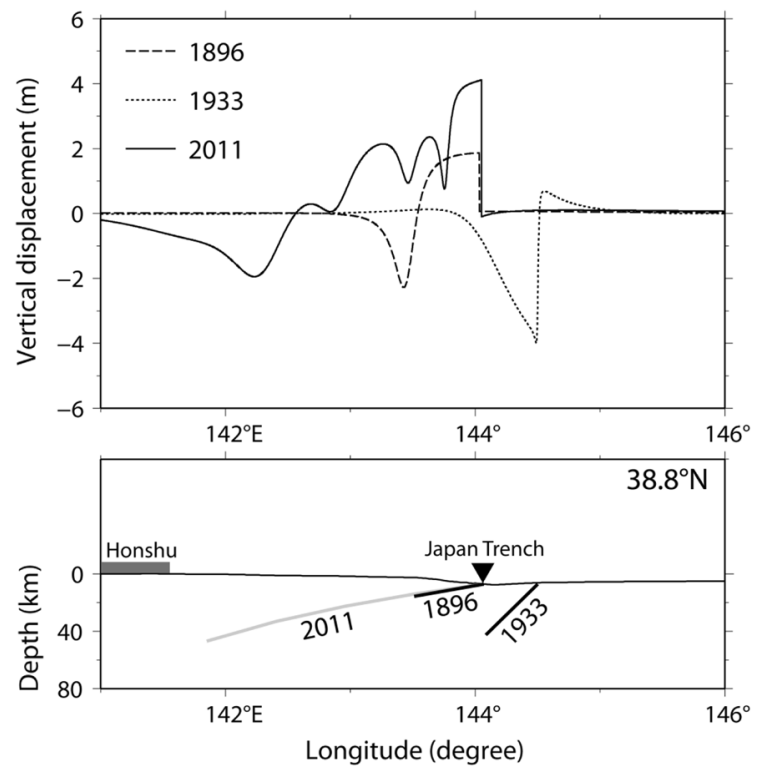

Figure 20

Seafloor deformation along the latitude of $38.8^{\circ} \mathrm{N}$ caused by the 1896 Sanriku (dashed curve), 1933 Sanriku (dotted curve), and 2011 Tohoku (solid curve) earthquakes. Cross sections of fault models for the 1896 Sanriku (TANIOKA and SENo 2001), 1933 Sanriku (AidA 1977), and 2011 Tohoku (SATAKE et al. 2013) earthquakes are shown below. Locations of trench axis and land area are also shown

The period of an incoming tsunami wave is also a controlling factor. The dominant period for the 2011 event was estimated to be $\sim 45 \mathrm{~min}$ at DART 21418 and 33-66 min at DART 21413 (BORRERO and GREER 2013), while Heidarzadeh and Satake (2013) estimated the two dominant periods of 37 and $67.4 \mathrm{~min}$ from multiple DART records. The dominant periods of the preceding two Sanriku tsunamis are expected to be shorter because their source dimensions were much smaller than that of the 2011 event (i.e., $210 \mathrm{~km} \times 50 \mathrm{~km}$ for the 1896 tsunami; TANIOKA and SATAKE 1996, and $185 \mathrm{~km} \times 50 \mathrm{~km}$ for the 1933 Sanriku tsunami; AIDA 1977). However, the dominant period of the 2010 Chilean tsunami was much longer ( 110 min) at DART 21413 (Borrero and GreER 2013). The dominant period becomes longer after a tsunami propagates the Pacific Ocean because of the dispersion effect (WATADA et al. 2013).

For local tsunamis, the large variation of tsunami heights along the Sanriku coast are probably caused by matching the periods of incoming waves and the characteristic periods of some bays. On the other hand, tsunami heights are less sensitive to the coastal topography and show a more uniform distribution for trans-Pacific Chilean tsunamis, resulting from longer periods than the characteristic periods of bays along the Sanriku coast.

On the northern (north of $40.2^{\circ} \mathrm{N}$ ) and southern (south of $39.0^{\circ} \mathrm{N}$ ) Sanriku coasts, the local variations in tsunami height are much smaller. The tsunami heights from the three earthquakes were similar on the northern Sanriku coast, while the 2011 heights were much larger than those of the 1896 or 1933 Sanriku tsunamis on the southern Sanriku coast. The distance from the tsunami source and the earthquake magnitude control the tsunami heights on these coasts. The distances to the northern Sanriku coasts from the three sources were similar, while the 2011 tsunami source is much closer to the southern Sanriku coast (Fig. 1).

SUPPASRI et al. (2013) performed regression analyses between the earthquake magnitude and the maximum tsunami heights based on the historical tsunami trace database and the field survey of the 2011 Tohoku earthquake in each tsunami-affected location. They claimed that the earthquake magnitude is a major controlling factor in determining the maximum tsunami heights. However, the examples of the 1896 and 2011 tsunami heights are clear counterevidence for magnitude dependence. CHOI et al. (2012) approximated the distribution of tsunami heights along the coast by simple log-normal distributions, suggesting that the tsunami heights are controlled only by the distance from the source. However, the distribution of tsunami heights along the Sanriku coast clearly demonstrates a significant contribution by other factors such as irregular coastal topography.

\section{Comparison of Tsunami Heights on Ibaraki and Chiba Coasts}

For the Pacific coasts of Ibaraki and Chiba prefectures (between $34.9^{\circ}$ and $36.9^{\circ} \mathrm{N}$ ), Tsus et al. (2011) measured 79 tsunami heights at 35 locations. Of these, tsunami heights from the 1960 and 2010 Chile earthquakes were also reported at 24 and 15 locations, respectively (Fig. 21; Table 2). The 1960 

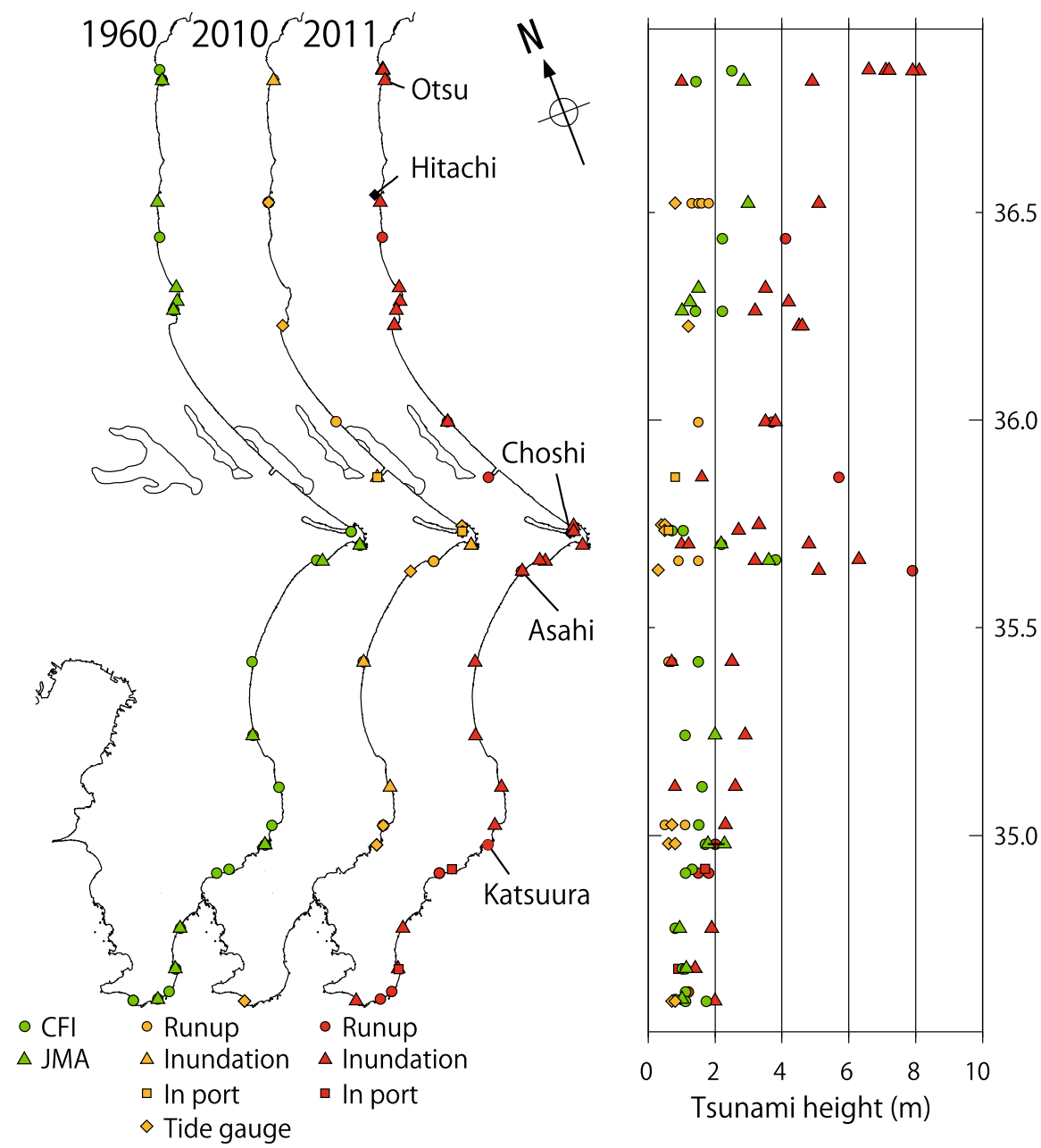

Figure 21

Comparison of 1960 and 2010 Chilean tsunami heights with 2011 Tohoku tsunami heights along the coasts of Ibaraki and Chiba prefectures. Green circles and triangles indicate 1960 heights from CFI (1961) and JMA (1961), respectively. Orange circles, triangles, squares, and diamonds, respectively, indicate runup heights, inundation heights, tsunami heights in ports, and tsunami heights from tide gauges from the 2010 Chile earthquake (Tsuj et al. 2010; Imai et al. 2010). Red circles, triangles, and squares, respectively, indicate 2011 runup heights, inundation heights, and tsunami heights in ports from Tsus et al. (2011). The measurement locations are indicated on the maps on the left.

Only data measured at the same locations for two or more tsunami are shown

tsunami heights are smaller than the 2011 tsunami heights (median ratio is 0.62 ; Table 3), and the 2010 heights are much smaller (median ratio 0.28).

\subsection{Tsunami Heights}

At Otsu in Kitaibaraki City, the measured inundation height from the 2011 Tohoku tsunami was $\sim 5 \mathrm{~m}$ (B6). The 1960 tsunami heights were $1 \mathrm{~m}$ (CFI 1961) or $3 \mathrm{~m}$ (JMA 1961), while the 2010 tsunami caused only a minor inundation with a height of $1 \mathrm{~m}$.
Along the coast of Ibaraki and Chiba prefectures, the 2011 tsunami heights gradually decreased toward the south. The two Chilean tsunamis also showed similar tendencies, though the change is smaller. These similar variations are reflected in the positive correlation of tsunami heights (Table 3; Fig. 22). The correlation coefficient between the 2011 and 1960 tsunami heights is 0.63 , and that for the 2011 and 2010 tsunamis is 0.41 .

Local amplifications of tsunami heights around Asahi City were found for the 2011, 2010, and 1960 


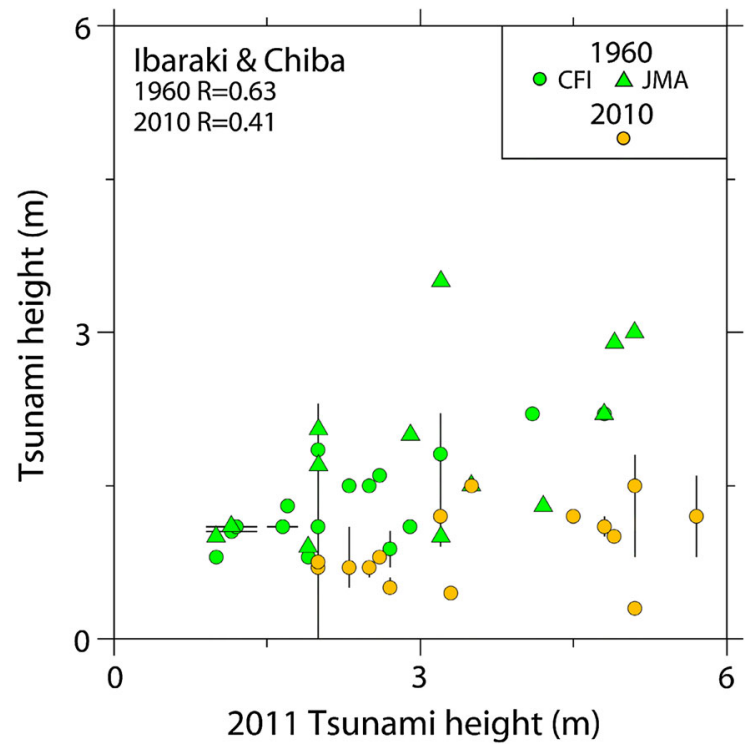

Figure 22

Comparison between the 2011 tsunami and the 1960 and 2010 Chilean tsunamis along the Ibaraki and Chiba coasts. Green circles and triangles indicate the 1960 Chilean tsunami heights from CFI (1961), and JMA (1961), respectively. Orange circles indicate the 2010 tsunami heights from Tsuj et al. (2010) or IMAI et al. (2010). Multiple height data at the same locations are represented as median values with ranges (shown as bars)

tsunamis: tsunami heights were locally high $(5-8 \mathrm{~m})$ around Asahi City in 2011, while the 1960 and 2010 Chilean tsunami heights were 4 and $2 \mathrm{~m}$, respectively. This local peak may be due to the local topography (the peninsula around Choshi and the local bathymetry off Asahi). The tsunami heights were also locally large around Katsuura, another gentle peninsula (Fig. 21). These common local variations also contributed to the large correlation coefficients.

\subsection{Factors Controlling Tsunami Heights and their Variability}

The 2011 tsunami heights generally decreased toward the south, away from the tsunami source. The 1960 and 2010 tsunamis, which were generated by the earthquakes in Chile $\left(M_{\mathrm{w}} 9.5\right.$ and 8.8, respectively) and propagated across the Pacific Ocean, show more uniform heights along the coasts, although they also decreased toward the south. Of the two Chilean tsunamis, the 2010 tsunami heights are consistently lower than the 1960 heights because of the smaller earthquake magnitude.
On a smaller scale, both near-field and transoceanic tsunamis show similar local variations. Local peaks around Choshi and Asahi, and near Katsuura were found for all the tsunamis, possibly as a result of the local topography, which consists of a number of small peninsulas. The peninsula is more distinct around Choshi and the tsunami heights show a more significant peak, while the peninsula near Katsuura is gentler and the peak in tsunami heights is less pronounced. This indicates that the local topography also affects local variation in tsunami height, although the main controlling factor is the source location, slip distribution, and the earthquake magnitude.

\section{Conclusions}

We summarized our 12 field surveys in which 296 tsunami heights accompanying the 2011 Tohoku earthquake were measured. The data and detailed locations of survey points and photographs (this paper and Tsus et al. 2011) will be useful for modeling the 2011 tsunami source (e.g., SATAKe et al. 2013). We then compared tsunami heights for the 2011 Tohoku earthquake with those from past earthquakes: the 1896 and 1933 Sanriku earthquakes in Japan, and the 1960 and 2010 Chile earthquakes. Along the central Sanriku coast (between $39.0^{\circ}$ and $40.2^{\circ} \mathrm{N}$ ), the 2011 and 1896 tsunami heights ranged from 5 to $40 \mathrm{~m}$, showing significant local variation. This may be due to the rugged and irregular coastline, indicating that local topography is a major factor in controlling tsunami height, together with the location, type, or magnitude of the earthquake. This is evident from the fact that the largest tsunami height was recorded at around $40.0^{\circ} \mathrm{N}$ for the 1896 and 2011 tsunamis, despite these tsunamis having different source locations. The local variations are much smaller on the northern and southern Sanriku coasts and the Ibaraki and Chiba coasts. The 2011 tsunami heights generally decrease toward the north and south, and also show local variations probably due to local topography. The 1960 and 2010 Chilean tsunami heights are more uniform. Both near-field and transoceanic tsunamis exhibit local peaks in their heights near peninsulas. Such local variations of 
tsunami heights may be helpful for educating coastal residents to reduce future tsunami disasters.

\section{Acknowledgments}

We thank Haeng Yoong Kim, Toshihiro Ueno, Satoko Murotani, Satoko Oki, Megumi Sugimoto, Jiro Tomari, Mohammad Heidarzadeh, Shingo Watada, Kentaro Imai, Byung Ho Choi, Sung Bum Yoon, Jae Seok Bae, Kyeong Ok Kim, Hyun Woo Kim, Makoto Yoshimizu, and Morio Koyama for helping to measure tsunami heights. We also thank Kentaro Imai for providing data on tsunami heights from the 2010 Chile earthquake. Valuable comments and suggestions from the guest editor, Dr. Jose C. Borrero, and two anonymous reviewers were very helpful in improving our manuscript. Most of the figures were generated using Generic Mapping Tools (Wessel and Sмith 1998). We also used a digital topographic map with a scale of 1:25,000 from the Geospatial Information Authority of Japan. This study was partially supported by Grants-in-Aid for Scientific Research from the Ministry of Education, Culture, Sports, Science and Technology, Japan.

Open Access This article is distributed under the terms of the Creative Commons Attribution License which permits any use, distribution, and reproduction in any medium, provided the original author(s) and the source are credited.

\section{REFERENCES}

ABE, K., 1994. Instrumental magnitudes of historical earthquakes, 1892 to 1898, Bulletin of the Seismological Society of America, $84,415-425$.

AIDA, I, 1977. Simulations of large tsunamis occurring in the past off the coast of the Sanriku district, Bulletin of the Earthquake Research Institute, University of Tokyo, 52, 71-101 (in Japanese with English abstract).

Borrero, J.C. and GreEr S.D., 2013. Comparison of the 2010 Chile and 2011 Japan tsunamis in the far-field, Pure and Applied Geophysics, 170, 1249-1274, doi:10.1007/s00024-012-0559-4.

CFI (Committee for Field Investigation of the Chilean Tsunami of 1960), 1961. Report on the Chilean tsunami of May 24, 1960, as observed along the coast of Japan, Tokyo, Maruzen Co., 397 pp.

Choi, B.H., Min, B.I., Pelinovsky, E., Tsuj,, Y., and Kim, K.O., 2012. Comparable analysis of the distribution functions of runup heights of the 1896, 1933 and 2011 Japanese Tsunamis in the Sanriku area, Natural Hazards and Earth System Sciences, 12, 1463-1467.
EARThQuake Research Institute, 1934. Field survey report of damage caused by the 1933 Sanriku earthquake, Bulletin of the Earthquake Research Institute, University Tokyo (supplementary volume), -Papers and Reports on the Tsunami of 1933 on the Sanriku Coast, Japan, 1, 9-139 (in Japanese).

Fuji, Y., Satake, K., Sakai, S., Shinohara, M., and Kanazawa, T., 2011. Tsunami source of the 2011 off the Pacific coast of Tohoku Earthquake, Earth, Planets and Space, 63, 815-820.

Grilli, S.T., Harris, J.C., Bakhsh, T.S.T., Masterlark, T.L., KyRiakopoulos, C., Kirby, J.T., and ShI, F., 2013. Numerical simulation of the 2011 Tohoku tsunami based on a new transient FEM co-seismic source: comparison to far- and near-field observations, Pure and Applied Geophysics, 170, 1333-1359, doi:10.1007/s00024-012-0528-y.

Haraguchi, T. and Inamatsu, A., 2011. Detailed maps of the impacts of the 2011 Japan Tsunami, Vol. 1: Aomori, Iwate and Miyagi prefectures, Kokon-Shoin, Publishers Ltd., Tokyo, 167 pp. (in Japanese).

Hatori, T., 1995. Review of documents for the 1896 Meiji Sanriku tsunami along the coast of Iwate Prefecture, Tsunami Engineering Technical Report, Disaster Control Research Center, Tohoku University, 12, 59-65 (in Japanese).

Honda, K., Terada, T., Yoshida, Y., and Isitani, D., 1908. Secondary undulations of oceanic tides, Journal of the College of Science, Imperial University, Tokyo, Japan, 24, 1-113.

IкI, T., 1897. A report of the field investigation of the tsunami of 1896 in the Sanriku District, Reports of the Imperial Earthquake Investigation Committee, 11, 5-34 (in Japanese).

Imai, K., Namegaya, Y., Tsuji, Y., Fuji, Y., Ando, R., Komatsubara, J., Komatsubara, T., Horikawa, H., Miyachi, Y., Matsuyama, M., YoshiI, T., Ishibe, T., Satake, K., Nishiyama, A., Harada, T., Shigihara, Y., Shigihara, Y., and Fujima, K., 2010. Field survey for tsunami trace height along the coasts of the Kanto and Tokai districts from the 2010 Chile Earthquake, Journal of Japan Society of Civil Engineers, Ser. B2 (Coastal Engineering), 66, 1351-1355 (in Japanese with English abstract).

Imamura, A., 1934. Past tsunamis of the Sanriku coast, Japanese Journal of Astronomy and Geophysics, 11, 79-93.

Imamura, F. and Watanabe, T., 1990. Surveys of large Sanriku tsunamis at Taro, Iwate Prefecture, Research Report of the Tsunami Disaster Prevention Laboratory, Civil Engineering, Tohoku University, 7, 123-140 (in Japanese).

JMA (Japan Meteorological Agency), 1961. Report on the tsunami of the Chilean earthquake, 1960, Technical Report of the Japan Meteorological Agency, 8, 389 pp. (in Japanese).

JMA (JaPAn Meteorological Agency), 2011. Monthly report on earthquakes and volcanoes in Japan March 2011, 321 pp. (in Japanese).

KANAMORI, H., 1971. Seismological evidence for a lithospheric normal faulting - the Sanriku Earthquake of 1933, Physics of the Earth and Planetary Interiors, 4, 289-300.

Kanamori, H., 1972. Mechanism of tsunami earthquakes, Physics of the Earth and Planetary Interiors, 6, 346-359.

Koike, K., Tamura, T., Chinzei, K., and Miyagi, T., editors, 2005. Regional Geomorphology of the Japanese Islands Vol. 3 Geomorphology of Tohoku Region, University of Tokyo Press, Tokyo, 355 pp. (in Japanese).

Kunitomi, S., 1933. Off Sanriku earthquake and tsunami on March 3, 1933, Quarterly Journal of Seismology, 7, 111-153.

Matsuo, H., 1933. Report on the survey of the 1933 Sanriku tsunami, Report of the Civil Engineering Laboratory, 24, 83-136 (in Japanese). 
Matsuo, H., 1934. Report on the survey of the 1933 Sanriku tsunami (supplement), Report of the Civil Engineering Laboratory, 27, 93-94 (in Japanese).

Heidarzadeh, M., and Satake, K., 2013. Waveform and spectral analyses of the 2011 Japan tsunami records on tide gauge and DART stations across the Pacific ocean, Pure and Applied Geophysics, 170, 1275-1293, doi:10.1007/s00024-012-0558-5.

Mori, N., Takahashi, T., Yasuda, T., and Yanagisawa, H., 2011. Survey of 2011 Tohoku earthquake tsunami inundation and runup, Geophysical Research Letters, 38, L00G14, doi:10.1029/ 2011GL049210.

Mori, N., Takahashi, T., and The 2011 Tohoku Earthquake Tsunami Joint Survey Group, 2012. Nationwide post event survey and analysis of the 2011 Tohoku earthquake tsunami, Coastal Engineering Journal, 54(01), 1250001, doi:10.1142/S057856341 2500015.

OкаL, E.A., 2013. From 3-Hz $P$ waves to ${ }_{0} S_{2}$ : no evidence of a slow component to the source of the 2011 Tohoku Earthquake, Pure and Applied Geophysics, 170, 963-973, doi:10.1007/s00024012-0500-X.

OtukA, Y., 1934. Tsunami damages March 3rd, 1933, and the topography of Sanriku coast, Japan, Bulletin of Earthquake Research Institute, University Tokyo (supplementary volume), Papers and Reports on the Tsunami of 1933 on the Sanriku Coast, Japan, 1, 127-151.

Ozawa, S., Nishimura, T., Suito, H., Kobayashi, T., Tobita, M., and IMAKIIRE, T., 2011. Coseismic and postseismic slip of the 2011 magnitude-9 Tohoku-Oki earthquake, Nature, 475, 373-376.

Satake, K., FujiI, Y., Harada, T., and Namegaya, Y., 2013. Time and space distribution of coseismic slip of the 2011 Tohoku earthquake as inferred from tsunami waveform data, Bulletin of the Seismological Society of America, 103, 1473-1492.

Shuto, N. and Goto, T., 1985a. Trace surveys of the large Sanriku tsunamis-Raga, Hiraiga, and Shimanokoshi in Tanohata Village, Omoto and Shimokonari in Iwaizumi Town, Research Report of the Tsunami Disaster Prevention Laboratory, Faculty of Civil Engineering, Tohoku University, 2, 39-45 (in Japanese).

Sнuto, N. and Goto, T., 1985b. Trace surveys of the large Sanriku tsunami-Okirai in Sanriku Town, Research Report of the Tsunami Disaster Prevention Laboratory, Faculty of Civil Engineering, Tohoku University, 2, 46-53 (in Japanese).

Shuto, N. and Unohana, M., 1984. Traces on the 1983 NihonkaiChubu earthquake, Research Report of the Tsunami Disaster Prevention Laboratory, Faculty of Civil Engineering, Tohoku University, 1, 88-267 (in Japanese).

Suppasri, A., Fukutani Y., Abe, Y., and Imamura, F., 2013. Relation between earthquake magnitude and tsunami height along the
Tohoku coast based on historical tsunami trace database and the 2011 Great East Japan tsunami, Report of Tsunami Engineering, 30, 37-49.

TAKEDA A., 1987. The tsunami stone at Raga-a result of leveling of the old tide trace due to the Meiji-Sanriku tsunami in 1896, Report of the National Research Center for Disaster Prevention, 39, 163-169 (in Japanese with English abstract).

TANIOKA, Y. and SATAKe, K., 1996. Fault parameters of the 1896 Sanriku tsunami earthquake estimated from tsunami numerical modeling, Geophysical Research Letters, 23, 1549-1552.

Tanioka, Y. and Seno, T., 2001. Sediment effect on tsunami generation of the 1896 Sanriku tsunami earthquake, Geophysical Research Letters, 28, 3389-3392.

Tsuj,, Y., Ohtoshi, K., Nakano, S., Nishimura, Y., Fujima, K., Imamura, F., Kakinuma, T., NaKamura, Y., Imai, K., Goto, K., Namegaya, Y., Suzuki, S., Shiroshita, H., and Matsuzaki, Y., 2010. Field investigation on the 2010 Chilean Earthquake Tsunami along the comprehensive coastal region in Japan, Journal of Japan Society of Civil Engineers, Ser, B2 (Coastal Engineering), 66, 1346-1350 (in Japanese with English abstract).

Tsuj,, Y., Satake, K., Ishibe, T., Kusumoto, S., Harada, T., NIshiyama, A., Kim, H.Y., Ueno, T., Murotani, S., Oki, S., Sugimoto, M., Tomari, J., Heidarzadeh, M., Watada, S., Imai, K., Сног, B.H., Yoon, S.B., BAE, J.S., Kıм, K.O., and Kıм, H.W., 2011. Field surveys of tsunami heights from the 2011 off the Pacific Coast of Tohoku, Japan Earthquake, Bulletin of Earthquake Research Institute, University of Tokyo, 86, 29-279 (in Japanese with English abstract).

Unohana, M. and Ota, T., 1988. Disaster records of Meiji Sanriku tsunami by Soshin Yamana, Research Report of the Tsunami Disaster Prevention Laboratory, Faculty of Civil Engineering, Tohoku University, 5, 57-379 (in Japanese).

Watada, S., Kusumoto, S., and SAtake, K., 2013. Cause of traveltime difference between observed and synthetic tsunami waveforms at distant locations, Abstract for IAHS-IAPSO-IASPEI Joint Assembly, SP1S1.03.

Wessel, P. and SMITH, W.H.F., 1998. New, improved version of the Generic Mapping Tools released, EOS Transactions American Geophysical Union, 79, 579.

Yamana, S., 1896. Disaster records of Meiji Sanriku tsunami, reproduced by Unohana and Ota (1988), Research Report of the Tsunami Disaster Prevention Laboratory, Faculty of Civil Engineering, Tohoku University, 5, 57-379 (in Japanese).

YAMAShita, F., 2003. Brief history of "declaration of tsunami mitigation town" and large breakwaters in Taro Town, Sanriku coast, Historical Earthquakes, 19, 165-171 (in Japanese). 